\title{
Angewandte
}

Supporting Information

\section{Total Synthesis of the Diterpenoid (+)-Harringtonolide}

Hai-Jun Zhang ${ }^{+}$Lin $\mathrm{Hu}^{+}$, Zhiqiang Ma, Ruining Li, Zhen Zhang, Cheng Tao, Bin Cheng, Yun Li, Huifei Wang, and Hongbin Zhai*

anie_201605879_sm_miscellaneous_information.pdf 


\section{Supporting Information}

\section{Table of Contents}

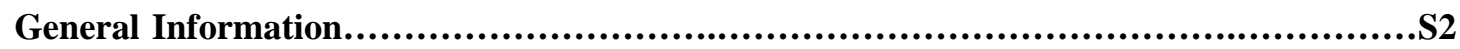

Experimental Procedures and Characterization Data for (+)-Harringtonolide ................S3

Comparison of Spectra of Natural and Synthetic Harringtonolide..........................S27

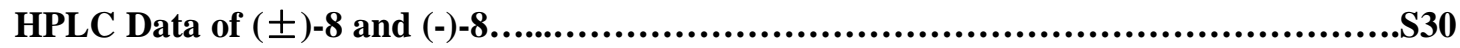

NMR Spectra of the Intermediates and (+)-Harringtonolide............................S32 


\section{General Information}

All reactions involving air or moisture sensitive reagents or intermediates were performed under an argon atmosphere with dry solvents under anhydrous conditions, unless otherwise noted. Methylene chloride $\left(\mathrm{CH}_{2} \mathrm{Cl}_{2}\right)$, diisopropylamine $\left(i-\mathrm{Pr}_{2} \mathrm{NH}\right)$, and triethylamine $\left(\mathrm{Et}_{3} \mathrm{~N}\right)$ were freshly distilled from calcium hydride. Toluene and THF were freshly distilled in the presence of the sodium/benzophenone couple. All reagents were reagent grade and used without purification unless otherwise noted. All extracts were dried over $\mathrm{MgSO}_{4}$ or $\mathrm{Na}_{2} \mathrm{SO}_{4}$ and concentrated by rotary evaporation below $30{ }^{\circ} \mathrm{C}$ unless otherwise noted. Proton nuclear magnetic resonance $\left({ }^{1} \mathrm{H}\right.$ NMR) and carbon nuclear magnetic resonance $\left({ }^{13} \mathrm{C}\right.$ NMR $)$ spectra were obtained at the indicated field as solutions in $\mathrm{CDCl}_{3}$. Chemical shifts are referenced to the deuterated solvent $\left(\mathrm{CDCl}_{3}, \delta=7.27 \mathrm{ppm}\right.$ and $77.0 \mathrm{ppm}$ for ${ }^{1} \mathrm{H}$ and ${ }^{13} \mathrm{C}$ NMR, respectively) and are reported in parts per million (ppm, $\delta$ ) relative to tetramethylsilane (TMS, $\delta=0.00 \mathrm{ppm}$ ). Signal splitting patterns were described as singlet (s), doublet (d), triplet (t), quartet (q), multiplet (m) or broad (br), and coupling constants (J) are reported in Hz. ${ }^{1} \mathrm{H}$ spectra were recorded on a Brucker Avance $400 \mathrm{III}$ spectrometer (400 MHz). ${ }^{13} \mathrm{C}$ NMR spectra were recorded on Brucker Avance 400 III spectrometer at $100 \mathrm{MHz}$. HPLC was recorded on a Waters 600/2996 spectrometer using CHIRALCEL OJ-H, Column No OJHOCE-EAO30. Optical rotations were recorded on a RUDOLPH A21202-T digital polarimeter at ambient temperature. High resolution mass spectra (HRMS) were performed by Analytical Instrument Center at the School of Pharmacy or Department of Chemistry of Lanzhou University on an Electron Spray Injection (ESI) mass spectrometer. Melting point was recorded on a SGWB X-4A melting point apparatus. X-ray diffraction was recorded on a Supernova apparatus. 


\section{Experimental Procedures and Characterization Data for (+)-Harringtonolide}

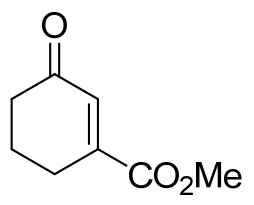

9

To a $1 \mathrm{~L}$ three-necked flask equipped with a stirrer, an ammonia $(\mathrm{g})$ outlet and a cold-finger condenser were added $m$-Anisic acid $(70 \mathrm{~g}, 0.46 \mathrm{~mol})$ and $\mathrm{H}_{2} \mathrm{O}(90 \mathrm{~mL})$. Ammonia $(800 \mathrm{~mL})$ was collected through the condenser containing $\mathrm{N}_{2}$ (1). Lithium wire (9.6 g, $1.4 \mathrm{~mol}$ ) was added in small pieces over a period of $30 \mathrm{~min}$ and the reaction mixture was stirred vigorously for $4 \mathrm{~h}$. The stirring was stopped and the solution was allowed to warm to room temperature overnight. Then $\mathrm{KOH}(\mathrm{aq}, 1.0 \mathrm{M}, 580 \mathrm{~mL}$ ) was added and stirring was restarted. The solution was heated for $3 \mathrm{~h}$ at $60{ }^{\circ} \mathrm{C}$ and then cooled to about $10{ }^{\circ} \mathrm{C}$. Concentrated $\mathrm{HCl}(72 \mathrm{~mL})$ was added at $0{ }^{\circ} \mathrm{C}$ until the $\mathrm{pH}=$ 2. The aqueous solution was extracted with $\mathrm{DCM} /{ }^{i} \mathrm{PrOH}=3: 1$. The combined organic layers were dried over $\mathrm{MgSO}_{4}$, filtered, and concentrated under vacuum. The crude product was used for the next step without further purification.

To a solution of the crude product in $\mathrm{MeOH}(400 \mathrm{~mL})$ was added $\mathrm{SOCl}_{2}(44 \mathrm{~g}, 0.37 \mathrm{~mol})$ at 0 ${ }^{\circ} \mathrm{C}$. The solution was allowed to warm to $\mathrm{rt}$ and stirred overnight. The mixture was concentrated under vacuum, diluted with EtOAc, washed with $\mathrm{NaHCO}_{3}$ (aq) and brine sequentially, dried over $\mathrm{Na}_{2} \mathrm{SO}_{4}$, filtered, and concentrated under vacuum. The crude product was used for the next step without further purification.

To a solution of the crude product in $\mathrm{CHCl}_{3}(858 \mathrm{~mL})$ was added $\mathrm{DBU}(56 \mathrm{~g}, 0.37 \mathrm{mmol})$ at 0 ${ }^{\circ} \mathrm{C}$. The solution was stirred at $\mathrm{rt}$ for $12 \mathrm{~h}$ and then washed with $1.0 \mathrm{M} \mathrm{HCl}$ (aq) and brine sequentially. The organic layer was dried over $\mathrm{Na}_{2} \mathrm{SO}_{4}$, filtered, and concentrated under vacuum. The residue was purified by flash column chromatography on silica gel (EtOAc/hexanes $=1: 3, R_{f}$ $=0.29)$ to give 9 ( $35 \mathrm{~g}, 50 \%$ over three steps) as a light yellow oil. 9: ${ }^{1} \mathrm{H}$ NMR (400 $\left.\mathrm{MHz}, \mathrm{CDCl}_{3}\right)$ $\delta 6.74(\mathrm{t}, J=2.0 \mathrm{~Hz} 1 \mathrm{H}), 3.83(\mathrm{~s}, 3 \mathrm{H}), 2.61-2.57(\mathrm{~m}, 2 \mathrm{H}), 2.47-2.44(\mathrm{~m}, 2 \mathrm{H}), 2.10-2.03(\mathrm{~m}$, $2 \mathrm{H}) ;{ }^{13} \mathrm{C}$ NMR $\left(100 \mathrm{MHz}, \mathrm{CDCl}_{3}\right) \delta 200.0,167.0,148.7,133.1,52.6,37.7,24.8,22.1$; HRMS (ESI): $m / z$ calcd for $\mathrm{C}_{8} \mathrm{H}_{11} \mathrm{O}_{3}[\mathrm{M}+\mathrm{H}]^{+}: 155.0703$, found: 155.0705 . 


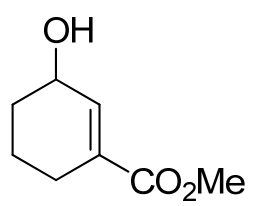

$( \pm)-8$

To a solution of $9(28.4 \mathrm{~g}, 184 \mathrm{mmol})$ in $\mathrm{MeOH}(367 \mathrm{~mL})$ was added $\mathrm{NaBH}_{4}(6.99 \mathrm{~g}, 184 \mathrm{mmol})$ at $0{ }^{\circ} \mathrm{C}$. The mixture was stirred for 10 min before it was quenched with $\mathrm{NH}_{4} \mathrm{Cl}$ (aq). The aqueous layer was extracted with EtOAc three times. The combined organic layers were dried over $\mathrm{MgSO}_{4}$, filtered, and concentrated under vacuum. The residue was purified by flash column chromatography on silica gel $\left(\right.$ EtOAc/hexanes $\left.=1: 3, R_{f}=0.22\right)$ to giv alcohol $( \pm)-8(27.8 \mathrm{~g}, 98 \%)$ as a light yellow oil. $( \pm)-8:{ }^{1} \mathrm{H}$ NMR $\left(400 \mathrm{MHz}, \mathrm{CDCl}_{3}\right) \delta 6.88(\mathrm{~d}, J=1.2 \mathrm{~Hz}, 1 \mathrm{H}), 4.36(\mathrm{~s}, 1 \mathrm{H})$, $3.76(\mathrm{~s}, 3 \mathrm{H}), 2.34-2.20(\mathrm{~m}, 2 \mathrm{H}), 1.99-1.91(\mathrm{~m}, 1 \mathrm{H}) 1.87-1.76(\mathrm{~m}, 1 \mathrm{H}) 1.68-1.53(\mathrm{~m}, 3 \mathrm{H})$; ${ }^{13} \mathrm{C}$ NMR (100 MHz, $\left.\mathrm{CDCl}_{3}\right) \delta 167.7,139.7,132.5,66.0,51.8,31.1,24.2,19.1 ;$ HRMS (ESI): $\mathrm{m} / \mathrm{z}$ calcd for $\mathrm{C}_{8} \mathrm{H}_{12} \mathrm{O}_{3} \mathrm{Na}[\mathrm{M}+\mathrm{Na}]^{+}:$179.0679, found: 179.0676 .

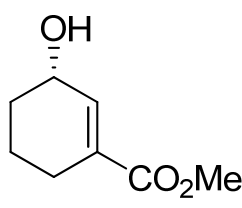

8

To a solution of $\mathbf{9}(10.2 \mathrm{~g}, \quad 66.2 \mathrm{mmol})$ in DCE $(200 \mathrm{~mL})$ were added $\mathrm{RuCl}\left(p\right.$-cymene) $\left[(S, S)\right.$-Ts-DPEN] $(210 \mathrm{mg}, 0.331 \mathrm{mmol}), \mathrm{NEt}_{3}(6.70 \mathrm{~g}, 66.2 \mathrm{mmol})$, and $\mathrm{HCO}_{2} \mathrm{H}$ (4.88 $\mathrm{g}, 106 \mathrm{mmol})$ sequentially at room temperature and the reaction was monitored by TLC. When the reaction was completed, the mixture (organic phase) was washed with $\mathrm{NaHCO}_{3}$ (aq), dried over $\mathrm{Na}_{2} \mathrm{SO}_{4}$, filtered, and concentrated under vacuum. The residue was purified by flash column chromatography on silica gel to give (-)-8 $(10.0 \mathrm{~g}, 97 \%, e e=94.0 \%)$ as a light brown oil. $[\alpha]_{\mathrm{D}}^{18.2}=-46.1\left(c \quad 1.28, \mathrm{CHCl}_{3}\right)$.

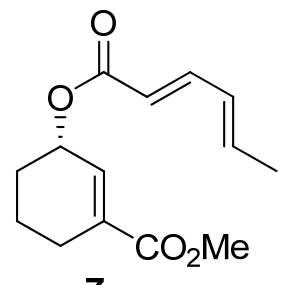

7

To a solution of alcohol $8(30.0 \mathrm{~g}, 192 \mathrm{mmol})$ in DCM $(550 \mathrm{~mL})$ were added sorbic acid $(27.6 \mathrm{~g}$, $246 \mathrm{mmol})$ and DMAP $(23.5 \mathrm{~g}, 192 \mathrm{mmol})$. After the solution was cooled to $0{ }^{\circ} \mathrm{C}$, DCC $(50.7 \mathrm{~g}$, 
$246 \mathrm{mmol}$ ) was added. The solution was allowed to warm to $\mathrm{rt}$ and stirred overnight at this temperature. The mixture was filtered using a Buchner funnel and washed with DCM three times. The filtrate was washed with $\mathrm{NaHCO}_{3}$ (aq) and $1.0 \mathrm{M} \mathrm{HCl}$ (aq) sequentially. The organic layer was dried over $\mathrm{Na}_{2} \mathrm{SO}_{4}$, filtered, and concentrated under vacuum. The residue was purified by flash column chromatography on silica gel $\left(\right.$ EtOAc/hexanes $\left.=1: 3, R_{\mathrm{f}}=0.77\right)$ to give $7(47.1 \mathrm{~g}$, 98\%) as a light yellow oil. 7: ${ }^{1} \mathrm{H}$ NMR $\left(400 \mathrm{MHz}, \mathrm{CDCl}_{3}\right) \delta 7.30-7.23(\mathrm{~m}, 1 \mathrm{H}), 6.85-6.84(\mathrm{~m}$, 1H), $6.23-6.12(\mathrm{~m}, 2 \mathrm{H}), 5.76(\mathrm{~d}, J=15.6 \mathrm{~Hz}, 1 \mathrm{H}), 5.49-5.46(\mathrm{~m}, 1 \mathrm{H}), 3.75(\mathrm{~s}, 3 \mathrm{H}), 2.40-2.22$ (m, 2H), $1.98-1.90(\mathrm{~m}, 1 \mathrm{H}), 1.86(\mathrm{~d}, J=5.2 \mathrm{~Hz}, 3 \mathrm{H}), 1.84-1.78(\mathrm{~m}, 1 \mathrm{H}), 1.74-1.64(\mathrm{~m}, 2 \mathrm{H})$; ${ }^{13} \mathrm{C}$ NMR $\left(100 \mathrm{MHz}, \mathrm{CDCl}_{3}\right) \delta 167.3,166.6,145.4,139.6,136.0,134.2,129.7,118.7,67.6,51.8$, 27.6, 24.1, 19.0, 18.6; HRMS (ESI): $m / z$ calcd for $\mathrm{C}_{14} \mathrm{H}_{18} \mathrm{O}_{4} \mathrm{Na}[\mathrm{M}+\mathrm{Na}]^{+}:$273.1097, found: $273.1095 ;[\alpha]_{\mathrm{D}}^{18.9}=-36.6\left(c 1.61, \mathrm{CHCl}_{3}\right)$.

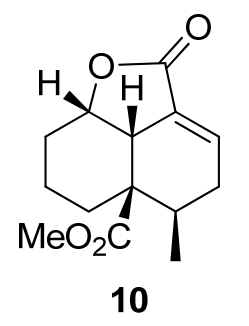

A mixture of $7(57.8 \mathrm{~g}, 231 \mathrm{mmol})$ and BHT $(5.09 \mathrm{~g}, 23.1 \mathrm{mmol})$ was heated at $180{ }^{\circ} \mathrm{C}$ for $24 \mathrm{~h}$, cooled to room temperature, and subjected to purification by flash column chromatography on silica gel $\left(\right.$ EtOAc/hexanes $\left.=1: 3, \mathbf{R}_{\mathrm{f}}=0.32\right)$ to give a mixture of $\mathbf{1 0}$ and the epimer 10' $(40.0 \mathrm{~g})$. The ${ }^{1} \mathrm{H}$ NMR spectra indicated that the ratio of $\mathbf{1 0}$ and $\mathbf{1 0}$ ' was 2.5:1.

The pure sample of $\mathbf{1 0}$ was obtained after $\mathbf{6}$ was separated in the next step. To a solution of $\mathbf{6}$ $(0.10 \mathrm{~g}, 0.40 \mathrm{mmol})$ in $\mathrm{CHCl}_{3}$ was added DBU $(0.091 \mathrm{~g})$. After $1 \mathrm{~h}$, the mixture was deluted with EtOAc and washed with $0.1 \mathrm{M} \mathrm{HCl}(\mathrm{aq})$. The organic layer was dried over $\mathrm{Na}_{2} \mathrm{SO}_{4}$, filtered, and concentrated under vacuum. The residue was purified by flash column chromatography on silica gel $\left(\right.$ EtOAc/hexanes $\left.=1: 3, R_{\mathrm{f}}=0.32\right)$ to give compound $\mathbf{1 0}$ as a light yellow oil $(0.095 \mathrm{~g}, 95 \%)$. 10: ${ }^{1} \mathrm{H}$ NMR (400 MHz, $\left.\mathrm{CDCl}_{3}\right) \delta 6.77(\mathrm{dd}, J=7.2,3.2 \mathrm{~Hz}, 1 \mathrm{H}), 4.94-4.88(\mathrm{~m}, 1 \mathrm{H}), 3.74(\mathrm{~s}, 3 \mathrm{H})$, $3.40-3.38(\mathrm{~m}, 1 \mathrm{H}), 2.62-2.54(\mathrm{~m}, 1 \mathrm{H}), 2.27-2.20(\mathrm{~m}, 1 \mathrm{H}), 2.19-2.11(\mathrm{~m}, 2 \mathrm{H}), 1.79(\mathrm{~d}, J=$ $13.2 \mathrm{~Hz}, 1 \mathrm{H}), 1.58-1.53(\mathrm{~m}, 1 \mathrm{H}), 1.25-1.18(\mathrm{~m}, 1 \mathrm{H}), 1.11-0.97(\mathrm{~m}, 2 \mathrm{H}), 0.82(\mathrm{~d}, J=7.2 \mathrm{~Hz}$, $3 \mathrm{H}) ;{ }^{13} \mathrm{C} \mathrm{NMR}\left(100 \mathrm{MHz}, \mathrm{CDCl}_{3}\right) \delta 176.0,169.7,132.8,126.7,76.9,52.2,49.6,37.0,32.8,30.6$, 
30.3, 29.0, 19.3, 17.0; HRMS (ESI): $m / z$ calcd for $\mathrm{C}_{14} \mathrm{H}_{19} \mathrm{O}_{4}[\mathrm{M}+\mathrm{H}]^{+}, 251.1278$, found: 251.1275; $[\alpha]_{\mathrm{D}}^{20.2}=-46.1\left(c 1.02, \mathrm{CHCl}_{3}\right)$.

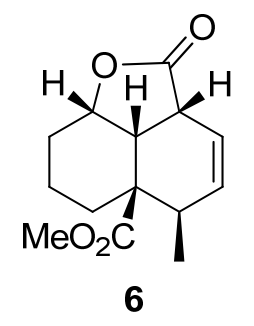

To a solution of freshly distilled diisopropylamine $(33.9 \mathrm{~mL}, 240 \mathrm{mmol})$ in dry THF (114 mL) was added $n$-BuLi $(2.5 \mathrm{M}$ in hexane, $80 \mathrm{~mL}, 200 \mathrm{mmol})$ dropwise at $-50{ }^{\circ} \mathrm{C}$. After $20 \mathrm{~min}$, the solution was cooled to $-78{ }^{\circ} \mathrm{C}$ and HMPA $(55.7 \mathrm{~mL}, 320 \mathrm{mmol})$ was added dropwise. After stirring for $10 \mathrm{~min}$, a solution of 10/10' (a mixture, $\left.20 \mathrm{~g}, 80 \mathrm{mmol}, \mathbf{1 0} / \mathbf{1 0}{ }^{\prime}=2.5: 1\right)$ in dry THF (40 $\mathrm{mL}$ ) was added dropwise. The reaction mixture was stirred at this temperature for $1 \mathrm{~h}$ before $\mathrm{AcOH}(30 \mathrm{~mL})$ was added as quickly as possible. The mixture was warmed to $\mathrm{rt}$ in $0.5 \mathrm{~h}$, diluted with EtOAc $(500 \mathrm{~mL})$, and washed with $\mathrm{NaHCO}_{3}$ (aq) until $\mathrm{pH}=9$ in aqueous. The organic layer was dried over $\mathrm{MgSO}_{4}$, filtered, and concentrated under vacuum. The residue was purified by flash column chromatography on silica gel (EtOAc/hexanes $\left.=1: 3, R_{\mathrm{f}}=0.38\right)$ to give compound 6 as a light yellow oil (10.2 g, $35 \%$ over two steps). 6: ${ }^{1} \mathrm{H}$ NMR (400 MHz, $\left.\mathrm{CDCl}_{3}\right) \delta 5.89-5.83$ (m, 2H), $4.93(\mathrm{dt}, J=6.4,1.6 \mathrm{~Hz}, 1 \mathrm{H}), 3.75(\mathrm{~s}, 3 \mathrm{H}), 3.57-3.52(\mathrm{~m}, 1 \mathrm{H}), 3.36(\mathrm{~d}, J=10.8 \mathrm{~Hz}, 1 \mathrm{H})$, $2.24-2.18(\mathrm{~m}, 1 \mathrm{H}), 1.95-1.89(\mathrm{~m}, 1 \mathrm{H}), 1.81-1.71(\mathrm{~m}, 1 \mathrm{H}), 1.70-1.60(\mathrm{~m}, 1 \mathrm{H}), 1.59-1.51(\mathrm{~m}$, $3 \mathrm{H}), 0.80(\mathrm{~d}, J=7.2 \mathrm{~Hz}, 3 \mathrm{H}) ;{ }^{13} \mathrm{C} \mathrm{NMR}\left(100 \mathrm{MHz}, \mathrm{CDCl}_{3}\right) \delta 176.9,176.6,131.3,119.9,77.8$, 52.1, 45.2, 39.1, 37.2, 32.7, 25.9, 22.0, 17.5, 14.3; HRMS (ESI): $m / z$ calcd for $\mathrm{C}_{14} \mathrm{H}_{18} \mathrm{O}_{4} \mathrm{Na}$ $[\mathrm{M}+\mathrm{Na}]^{+}, 273.1097$, found: $273.1093 ;[\alpha]_{\mathrm{D}}^{18.6}=-54.1\left(c 1.09, \mathrm{CHCl}_{3}\right)$.

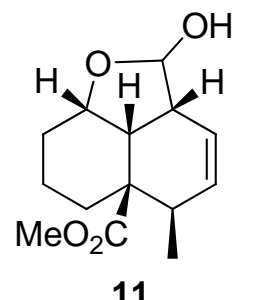

11

To a solution of $6(5.12 \mathrm{~g}, 20.5 \mathrm{mmol})$ in dry THF $(100 \mathrm{~mL})$ was added Dibal-H $(1.0 \mathrm{M}$ in toluene, $24.6 \mathrm{~mL}, 24.6 \mathrm{mmol}$ ) at $-78{ }^{\circ} \mathrm{C}$. The mixture was gradually warmed to $-40{ }^{\circ} \mathrm{C}$ over $3 \mathrm{~h}$ and then the reaction was quenched with saturated potassium sodium tartrate at $-78{ }^{\circ} \mathrm{C}$. The mixture was warmed to room temperature and stirred for additional $2 \mathrm{~h}$. The organic layer was 
separated and the aqueous layer was extracted with DCM. The combined organic layers were dried over $\mathrm{MgSO}_{4}$, filtered, and concentrated under vacuum. The residue was purified by flash column chromatography on silica gel (EtOAc/hexanes $\left.=1: 3, \mathrm{R}_{\mathrm{f}}=0.27\right)$ to give $\mathbf{1 1}(5.0 \mathrm{~g}, 97 \%)$ as a colorless oil that contains another unseparated diastereomer in a 1:10 ratio. 11: ${ }^{1} \mathrm{H}$ NMR (400 $\left.\mathrm{MHz}, \mathrm{CDCl}_{3}\right) \delta 5.81-5.73(\mathrm{~m}, 2 \mathrm{H}), 5.24(\mathrm{t}, J=2.8 \mathrm{~Hz}, 1 \mathrm{H}), 4.70-4.66(\mathrm{~m}, 1 \mathrm{H}), 3.71(\mathrm{~s}, 3 \mathrm{H})$, $3.34-3.24(\mathrm{~m}, 2 \mathrm{H}), 2.85(\mathrm{dd}, J=10.0,2.4 \mathrm{~Hz}, 1 \mathrm{H}), 2.27-2.21(\mathrm{~m}, 1 \mathrm{H}), 1.88-1.64(\mathrm{~m}, 2 \mathrm{H})$, $1.63-1.55(\mathrm{~m}, 3 \mathrm{H}), 1.50-1.40(\mathrm{~m}, 1 \mathrm{H}), 0.84(\mathrm{~d}, J=6.8 \mathrm{~Hz}, 3 \mathrm{H}) ;{ }^{13} \mathrm{C} \mathrm{NMR}\left(100 \mathrm{MHz}, \mathrm{CDCl}_{3}\right) \delta$ 177.5, 131.4, 124.7, 103.1, 76.9, 51.8, 46.8, 46.4, 37.0, 36.7, 27.7, 23.2, 16.9, 15.5; HRMS (ESI): $m / z$ calcd for $\mathrm{C}_{14} \mathrm{H}_{20} \mathrm{O}_{4} \mathrm{Na}[\mathrm{M}+\mathrm{Na}]^{+}, 275.1254$, found: $275.1256 ;[\alpha]_{\mathrm{D}}{ }^{18.8}=-32.4\left(c 1.82, \mathrm{CHCl}_{3}\right)$.

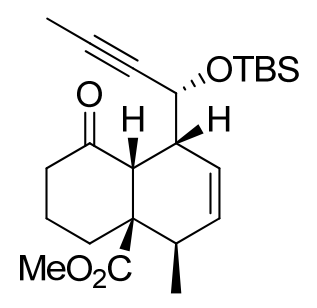

14

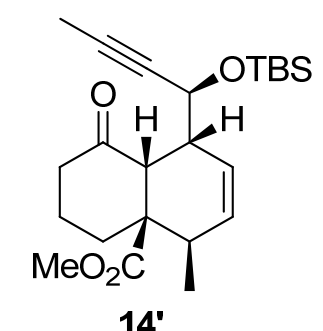

14

A solution of 1-propynylmagnesium bromide ( $0.5 \mathrm{M}$ in THF, $114 \mathrm{~mL}, 57 \mathrm{mmol})$ was added to $11(4.99 \mathrm{~g}, 19.8 \mathrm{mmol})$ at $0{ }^{\circ} \mathrm{C}$. The mixture was heated at reflux for $36 \mathrm{~h}$. The reaction was quenched with $\mathrm{NH}_{4} \mathrm{Cl}$ (aq) at $0{ }^{\circ} \mathrm{C}$ and extracted with DCM three times. The combined organic layers were dried over $\mathrm{MgSO}_{4}$, filtered, and concentrated under vacuum to give the crude product as a yellow foam that required no further purification.

To a solution of the crude product in dry DMF $(40 \mathrm{~mL})$ were added imidazole $(5.39 \mathrm{~g}, 79.2$ mmol) and $\mathrm{TBSCl}(5.94 \mathrm{~g}, 39.6 \mathrm{mmol})$ at room temperature. After being stirred for $12 \mathrm{~h}$, the mixture was diluted with $\mathrm{Et}_{2} \mathrm{O}(300 \mathrm{~mL})$, washed with $\mathrm{H}_{2} \mathrm{O}$ three times, dried over $\mathrm{MgSO}_{4}$, filtered, and concentrated under vacuum. The residue was purified by flash column chromatography on silica gel (EtOAc/hexanes $\left.=1: 10, \mathrm{R}_{\mathrm{f}}=0.47\right)$ to give a mixture of the product.

To a solution of the above crude product in dry DCM $(40 \mathrm{~mL})$ were added DMP $(13.0 \mathrm{~g}, 30.7$ mmol) and $\mathrm{NaHCO}_{3}(4.99 \mathrm{~g}, 59.4 \mathrm{mmol})$ at room temperature. The mixture was stirred for $24 \mathrm{~h}$ before quenching with $\mathrm{NaHCO}_{3}$ (aq) and $\mathrm{Na}_{2} \mathrm{~S}_{2} \mathrm{O}_{3}$ (aq) at $0{ }^{\circ} \mathrm{C}$. The mixture was extracted with $\mathrm{Et}_{2} \mathrm{O}$. The combined organic layers were dried with $\mathrm{MgSO}_{4}$, filtered, and concentrated under vacuum. The residue was purified by flash column chromatography on silica gel to give the 
products $14\left(2.6 \mathrm{~g}, 32 \%\right.$ over three steps, EtOAc/hexanes $\left.=1: 10, \mathrm{R}_{\mathrm{f}}=0.47\right)$ and $14^{\prime}(2.8 \mathrm{~g}, 35 \%$ over three steps, EtOAc/hexanes $\left.=1: 10, \mathrm{R}_{\mathrm{f}}=0.36\right)$ as light yellow oil. 14: ${ }^{1} \mathrm{H}$ NMR $(400 \mathrm{MHz}$, $\left.\mathrm{CDCl}_{3}\right) \delta 5.70(\mathrm{~d}, J=10.0 \mathrm{~Hz}, 1 \mathrm{H}), 5.49(\mathrm{dt}, J=10.4,2.4 \mathrm{~Hz}, 1 \mathrm{H}), 4.88(\mathrm{dd}, J=10.8,2.4 \mathrm{~Hz}, 1 \mathrm{H})$, $3.71(\mathrm{~s}, 3 \mathrm{H}), 3.34(\mathrm{~d}, J=4.4 \mathrm{~Hz}, 1 \mathrm{H}), 2.45-2.34(\mathrm{~m}, 2 \mathrm{H}), 2.31-2.27(\mathrm{~m}, 3 \mathrm{H}), 2.10-2.03(\mathrm{~m}$, 2H), $1.91-1.82(\mathrm{~m}, 1 \mathrm{H}), 1.79(\mathrm{~d}, J=4.4 \mathrm{~Hz}, 3 \mathrm{H}), 1.17$ (d, $J=7.2 \mathrm{~Hz}, 3 \mathrm{H}), 0.86(\mathrm{~s}, 9 \mathrm{H}) ; 0.12$ (s, 3H), 0.01 (s, 3H); ${ }^{13} \mathrm{C}$ NMR (100 MHz, $\left.\mathrm{CDCl}_{3}\right) \delta 209.1,173.3,131.0,124.0,81.2,80.6,63.1$, 54.7, 51.9, 51.6, 44.2, 42.3, 31.5, 30.9, 25.8, 24.0, 18.0, 14.6, 3.5, - 4.3, - 5.4; HRMS (ESI): $\mathrm{m} / \mathrm{z}$ calcd for $\mathrm{C}_{23} \mathrm{H}_{36} \mathrm{O}_{4} \mathrm{NaSi}[\mathrm{M}+\mathrm{Na}]^{+}, 427.2275$, found: 427.2283; $[\alpha]_{\mathrm{D}}^{20.2}=-35.4\left(c 0.96, \mathrm{CHCl}_{3}\right)$. 14': ${ }^{1} \mathrm{H}$ NMR (400 MHz, $\left.\mathrm{CDCl}_{3}\right) \delta 5.74(\mathrm{~d}, J=10.4 \mathrm{~Hz}, 1 \mathrm{H}), 5.47(\mathrm{~d}, J=10.4 \mathrm{~Hz}, 1 \mathrm{H}), 4.90(\mathrm{dd}$, $J=9.6,2.0 \mathrm{~Hz}, 1 \mathrm{H}), 3.70(\mathrm{~s}, 3 \mathrm{H}), 3.42(\mathrm{~d}, J=4.4 \mathrm{~Hz}, 1 \mathrm{H}), 2.44(\mathrm{td}, J=12.8,6.8 \mathrm{~Hz}, 1 \mathrm{H}), 2.36-$ $2.24(\mathrm{~m}, 4 \mathrm{H}), 2.11-2.05(\mathrm{~m}, 2 \mathrm{H}), 1.90-1.75(\mathrm{~m}, 1 \mathrm{H}), 1.79$ (d, $J=2.0 \mathrm{~Hz}, 3 \mathrm{H}), 1.15(\mathrm{~d}, J=7.2$ $\mathrm{Hz}, 3 \mathrm{H}), 0.91$ (s, 9H); 0.14 (s, 3H), 0.13 (s, 3H); $\left.{ }^{13} \mathrm{C} \mathrm{NMR} \mathrm{(100} \mathrm{MHz,} \mathrm{CDCl}_{3}\right) \delta$ 209.2, 173.4, 130.6, 124.6, 81.7, 79.2, 63.9, 54.9, 54.3, 52.0, 43.7, 42.0, 31.3, 30.8, 25.9, 23.8, 18.2, 14.7, 3.5, 4.5, - 5.0; HRMS (ESI): $m / z$ calcd for $\mathrm{C}_{23} \mathrm{H}_{36} \mathrm{O}_{4} \mathrm{NaSi}[\mathrm{M}+\mathrm{Na}]^{+}, 427.2275$, found: 427.2280; $[\alpha]_{\mathrm{D}}^{19.9}=-104.8\left(c 1.04, \mathrm{CHCl}_{3}\right)$.

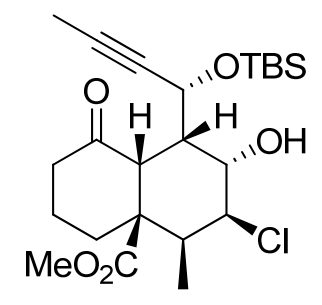

15

To a solution of $14(573 \mathrm{mg}, 1.42 \mathrm{mmol})$ in acetone $(7.1 \mathrm{~mL})$ was added $(\mathrm{CONCl})_{3}(263 \mathrm{mg}$, $1.14 \mathrm{mmol}$ ) at $0{ }^{\circ} \mathrm{C}$. After being stirred for $0.5 \mathrm{~h}$, the mixture was quenched with $\mathrm{NaHSO}_{3}(\mathrm{aq})$ and extracted with EtOAc. The combined organic layers were dried over $\mathrm{Na}_{2} \mathrm{SO}_{4}$, filtered, and concentrated under vacuum. The residue was purified by flash column chromatography on silica gel $\left(\right.$ EtOAc/hexanes $\left.=1: 8, R_{\mathrm{f}}=0.56\right)$ to give compound $15(401 \mathrm{mg}, 62 \%)$ as a colorless foam. 15 : ${ }^{1} \mathrm{H}$ NMR $\left(400 \mathrm{MHz}, \mathrm{CDCl}_{3}\right) \delta 5.52(\mathrm{~d}, J=8.0 \mathrm{~Hz}, 1 \mathrm{H}), 4.80(\mathrm{dd}, J=10.8,2.0 \mathrm{~Hz}, 1 \mathrm{H}), 4.26-$ $4.23(\mathrm{~m}, 1 \mathrm{H}), 4.05(\mathrm{t}, J=2.4 \mathrm{~Hz}, 1 \mathrm{H}), 4.01(\mathrm{~d}, J=4.4 \mathrm{~Hz}, 1 \mathrm{H}), 3.73(\mathrm{~s}, 3 \mathrm{H}), 2.80(\mathrm{dt}, J=10.8,4.0$ $\mathrm{Hz}, 1 \mathrm{H}), 2.51-2.44(\mathrm{~m}, 1 \mathrm{H}), 2.36-2.30(\mathrm{~m}, 1 \mathrm{H}), 2.22-2.16(\mathrm{~m}, 1 \mathrm{H}), 2.10-1.95(\mathrm{~m}, 3 \mathrm{H}), 1.84$ (d, $J=2.0 \mathrm{~Hz}, 3 \mathrm{H}), 1.82-1.75$ (m, 1H), 1.31 (d, $J=6.8 \mathrm{~Hz}, 3 \mathrm{H}), 0.89$ (s, 9H), 0.17 (s, 3H), 0.03 
$(\mathrm{s}, 3 \mathrm{H}) ;{ }^{13} \mathrm{C} \mathrm{NMR}\left(100 \mathrm{MHz}, \mathrm{CDCl}_{3}\right) \delta 218.8,174.9,82.3,78.9,71.1,67.2,61.7,52.1,52.0,50.7$, 42.0, 41.2, 34.9, 34.8, 25.8, 22.1, 18.0, 14.5, 3.6,-4.2,-5.3; HRMS (ESI): $\mathrm{m} / z$ calcd for $\mathrm{C}_{23} \mathrm{H}_{37} \mathrm{ClO}_{5} \mathrm{NaSi}[\mathrm{M}+\mathrm{Na}]^{+}, 479.1991$, found: $479.2002 ;[\alpha]_{\mathrm{D}}^{20.1}=+3.5\left(c 1.16, \mathrm{CHCl}_{3}\right)$.

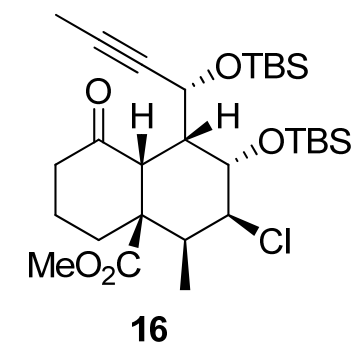

To a solution of $15(1.69 \mathrm{~g}, 3.71 \mathrm{mmol})$ in DCM $(19 \mathrm{~mL})$ were added $\mathrm{Et}_{3} \mathrm{~N}(750 \mathrm{mg}, 7.42 \mathrm{mmol})$ and TBSOTf $(1.47 \mathrm{~g}, 5.57 \mathrm{mmol})$ at $0{ }^{\circ} \mathrm{C}$. After the starting material disappeared as monitored by TLC, the mixture was quenched with $\mathrm{H}_{2} \mathrm{O}$ and extracted with EtOAc. The combined organic layers were dried over $\mathrm{Na}_{2} \mathrm{SO}_{4}$, filtered, and concentrated under vacuum. The residue was purified by flash column chromatography on silica gel (EtOAc/hexanes $\left.=1: 10, \mathrm{R}_{\mathrm{f}}=0.65\right)$ to give compound $16(1.5 \mathrm{~g}, 71 \%)$ as a colorless foam. 16: ${ }^{1} \mathrm{H}$ NMR $\left(400 \mathrm{MHz}, \mathrm{CDCl}_{3}\right) \delta 5.43(\mathrm{dd}, J=$ 10.8, $2.0 \mathrm{~Hz}, 1 \mathrm{H}), 4.29(\mathrm{t}, J=2.4 \mathrm{~Hz}, 1 \mathrm{H}), 3.87(\mathrm{t}, J=2.4 \mathrm{~Hz}, 1 \mathrm{H}), 3.69(\mathrm{~s}, 3 \mathrm{H}), 3.40(\mathrm{~d}, J=4.8$ $\mathrm{Hz}, 1 \mathrm{H}), 2.67$ (ddd, $J=10.4,4.8,2.0 \mathrm{~Hz}, 1 \mathrm{H}), 2.62-2.56$ (m, 1H), $2.37-2.26$ (m, 2H), 2.20 $2.12(\mathrm{~m}, 1 \mathrm{H}), 1.88(\mathrm{~s}, 3 \mathrm{H}), 1.87-1.68(\mathrm{~m}, 3 \mathrm{H}), 1.33$ (d, $J=7.2 \mathrm{~Hz}, 3 \mathrm{H}), 0.87$ (s, 9H), $0.86(\mathrm{~s}, 9 \mathrm{H})$, 0.17 (s, 3H), 0.16 (s, 3H) 0.07 (s, 3H), 0.05 (s, 3H); $\left.{ }^{13} \mathrm{C} \mathrm{NMR} \mathrm{(100} \mathrm{MHz,} \mathrm{CDCl}_{3}\right) \delta ;$ 207.7, 175.7, $81.8,80.7,72.3,67.4,62.9,51.8,49.5,48.3,43.3,40.6,33.9,33.3,25.8,25.7,18.9,18.1,17.9$, 14.6, 3.5, - 4.3, - 4.8, - 5.3, - 5.6; HRMS (ESI): $m / z$ calcd for $\mathrm{C}_{29} \mathrm{H}_{51} \mathrm{ClO}_{5} \mathrm{NaSi}_{2}[\mathrm{M}+\mathrm{Na}]^{+}$, 593.2856, found: $593.2865 ;[\alpha]_{\mathrm{D}}^{20.0}=+55.5\left(c 1.55, \mathrm{CHCl}_{3}\right)$.

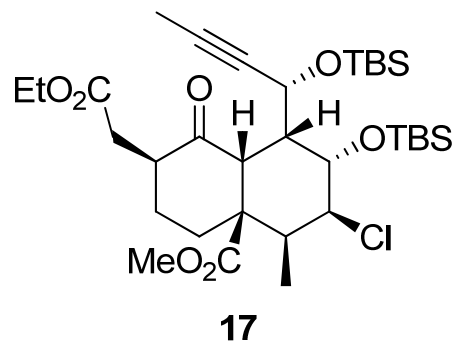

To a solution of freshly distilled diisopropylamine $(0.66 \mathrm{~g}, 6.5 \mathrm{mmol})$ in dry THF $(13 \mathrm{~mL})$ was added a solution of $n$-BuLi $(2.4 \mathrm{M}$ in hexane, $2.2 \mathrm{~mL}, 5.2 \mathrm{mmol})$ dropwise at $-50{ }^{\circ} \mathrm{C}$. After $0.5 \mathrm{~h}$, the solution was cooled to $-78{ }^{\circ} \mathrm{C}$ and then a solution of $\mathbf{1 6}(1.5 \mathrm{~g}, 2.6 \mathrm{mmol})$ in dry THF $(13 \mathrm{~mL})$ was added dropwise. After additional $1 \mathrm{~h}$, HMPA (1.4 g, $7.8 \mathrm{mmol})$ was added dropwise at this 
temperature. The resulting solution was stirred for $10 \mathrm{~min}$ and $\mathrm{BrCH}_{2} \mathrm{CO}_{2} \mathrm{Et}(0.65 \mathrm{~g}, 3.9 \mathrm{mmol})$ was added dropwise. The mixture was allowed to warm to $-40{ }^{\circ} \mathrm{C}$ and stirred for additional $1 \mathrm{~h}$ at this temperature. The mixture was quenched with $\mathrm{NH}_{4} \mathrm{Cl}$ (aq) at that temperature and extracted with EtOAc. The combined organic layers were washed with brine, dried over $\mathrm{Na}_{2} \mathrm{SO}_{4}$, filtered, and concentrated under vacuum. The residue was purified by flash column chromatography on silica gel $\left(\right.$ EtOAc/hexanes $\left.=1: 10, \mathrm{R}_{\mathrm{f}}=0.57\right)$ to give compound $17(0.92 \mathrm{~g}, 54 \%)$ as a colorless oil along with $0.46 \mathrm{~g}(31 \%)$ of recovered starting material. 17: ${ }^{1} \mathrm{H}$ NMR $\left(400 \mathrm{MHz}, \mathrm{CDCl}_{3}\right) \delta 5.15$ (dd, $J=10.4,2.0 \mathrm{~Hz}, 1 \mathrm{H}), 4.34(\mathrm{~s}, 1 \mathrm{H}), 4.13-4.07$ (m, 2H), $3.91(\mathrm{~s}, 1 \mathrm{H}), 3.68(\mathrm{~s}, 3 \mathrm{H}), 3.37$ (d, $J$ $=5.2 \mathrm{~Hz}, 1 \mathrm{H}), 2.88(\mathrm{brs}, 1 \mathrm{H}), 2.76-2.72(\mathrm{~m}, 2 \mathrm{H}), 2.64(\mathrm{~d}, J=16.0 \mathrm{~Hz}, 1 \mathrm{H}), 2.43(\mathrm{dd}, J=16.4$, $9.2 \mathrm{~Hz}, 1 \mathrm{H}), 2.25-2.12(\mathrm{~m}, 2 \mathrm{H}), 1.83(\mathrm{~d}, J=2.0 \mathrm{~Hz}, 3 \mathrm{H}), 1.81-1.76(\mathrm{~m}, 1 \mathrm{H}), 1.60$ (brs, $1 \mathrm{H})$, $1.37(\mathrm{~d}, J=6.4 \mathrm{~Hz}, 3 \mathrm{H}), 1.23$ (t, $J=7.2 \mathrm{~Hz}, 3 \mathrm{H}), 0.90$ (s, 9H), 0.83 (s, 9H), 0.20 (s, 3H), 0.14 (s, 3H), 0.10 (s, 3H), 0.07 (s, 3H); ${ }^{13} \mathrm{C}$ NMR (100 MHz, $\left.\mathrm{CDCl}_{3}\right) \delta 209.2,175.3,172.1,82.3,80.5$, $72.8,66.8,62.8,60.4,51.9,47.9,46.7,44.5,42.3,36.6,33.8,31.5,26.0,25.7,23.3,18.2,17.9$, 15.0, 14.2, 3.5, - 4.2, - 4.9, - 5.1, - 5.1; HRMS (ESI): $m / z$ calcd for $\mathrm{C}_{33} \mathrm{H}_{57} \mathrm{ClO}_{7} \mathrm{NaSi}_{2}[\mathrm{M}+\mathrm{Na}]^{+}$, 679.3224, found: $679.3233 ;[\alpha]_{\mathrm{D}}^{20.0}=+5.7\left(c 1.22, \mathrm{CHCl}_{3}\right)$.

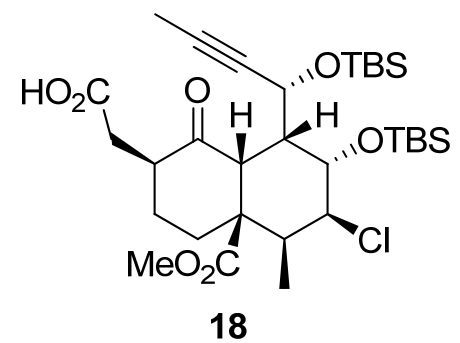

To a solution of the crude $17(0.92 \mathrm{~g}, 1.4 \mathrm{mmol})$ in $\mathrm{THF} / \mathrm{H}_{2} \mathrm{O}(4: 1,14 \mathrm{~mL})$ was added $\mathrm{LiOH}$ (0.29 g, $7.1 \mathrm{mmol})$. The mixture was stirred vigorously at $35{ }^{\circ} \mathrm{C}$ for $30 \mathrm{~h}$, diluted with DCM, washed with $1.0 \mathrm{M} \mathrm{HCl}$ (aq), dried over $\mathrm{MgSO}_{4}$, filtered, and concentrated under vacuum. The residue was purified by flash column chromatography on silica gel (EtOAc/hexanes $=1: 4, R_{f}=$ $0.35)$ to give compound $\mathbf{1 8}(0.33 \mathrm{~g}, 38 \%)$ as a colorless solid that contains another unseparated diastereomer in a 1:10 ratio along with $0.36 \mathrm{~g}(39 \%)$ of recovered starting material. 18: ${ }^{1} \mathrm{H}$ NMR $\left(400 \mathrm{MHz}, \mathrm{CDCl}_{3}\right) \delta 5.12(\mathrm{~d}, J=9.6 \mathrm{~Hz}, 1 \mathrm{H}), 4.34(\mathrm{~s}, 1 \mathrm{H}), 3.91(\mathrm{~s}, 1 \mathrm{H}), 3.69(\mathrm{~s}, 3 \mathrm{H}), 3.39(\mathrm{~d}, J=$ $5.2 \mathrm{~Hz}, 1 \mathrm{H}), 2.88(\mathrm{brs}, 1 \mathrm{H}), 2.74-2.70(\mathrm{~m}, 3 \mathrm{H}), 2.53-2.46(\mathrm{~m}, 1 \mathrm{H}), 2.26-2.18(\mathrm{~m}, 2 \mathrm{H}), 1.88-$ $1.75(\mathrm{~m}, 1 \mathrm{H}), 1.84(\mathrm{~d}, J=1.2 \mathrm{~Hz}, 3 \mathrm{H}), 1.62(\mathrm{brs}, 1 \mathrm{H}), 1.38$ (d, $J=5.6 \mathrm{~Hz}, 3 \mathrm{H}), 0.90$ (s, 9H), 0.83 
(s, 9H), $0.20(\mathrm{~s}, 3 \mathrm{H}), 0.15$ (s, 3H), $0.10(\mathrm{~s}, 3 \mathrm{H}), 0.07$ (s, 3H); ${ }^{13} \mathrm{C} \mathrm{NMR}\left(100 \mathrm{MHz}, \mathrm{CDCl}_{3}\right) \delta 209.3$, 177.6, 175.2, 82.4, 80.4, 72.8, 66.7, 62.8, 51.9, 47.8, 46.8, 44.6, 41.9, 36.3, 33.9, 31.6, 25.9, 25.7, 23.4, 18.2, 18.0, 15.0, 3.5, - 4.2, - 4.9, - 5.1, - 5.2; HRMS (ESI): $\mathrm{m} / z$ calcd for $\mathrm{C}_{31} \mathrm{H}_{53} \mathrm{ClO}_{7} \mathrm{NaSi}_{2}$ $[\mathrm{M}+\mathrm{Na}]^{+}, 651.2911$, found: $651.2907 ;[\alpha]_{\mathrm{D}}^{19.7}=+9.0\left(\right.$ c 1.22, $\left.\mathrm{CHCl}_{3}\right)$; $\mathrm{mp}=59-61^{\circ} \mathrm{C}$.

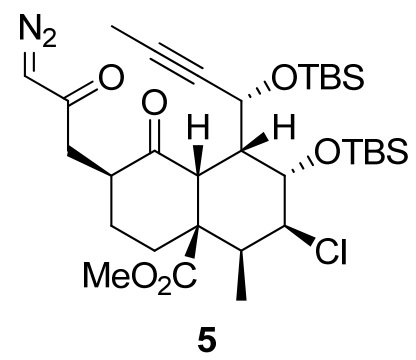

Preparation of $\mathrm{CH}_{2} \mathrm{~N}_{2}$ : Under continuously shaking, $\mathrm{CH}_{3} \mathrm{~N}(\mathrm{NO}) \mathrm{CONH}_{2}(2.6 \mathrm{~g}, 25 \mathrm{mmol})$ was added to a mixture of $\mathrm{KOH}\left(40 \%\right.$ in $\left.\mathrm{H}_{2} \mathrm{O}, 8.6 \mathrm{~mL}\right)$ and $\mathrm{Et}_{2} \mathrm{O}(26 \mathrm{~mL})$ at $0{ }^{\circ} \mathrm{C}$. Decantation of the

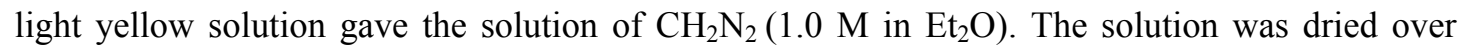
dusty $\mathrm{KOH}$ and used for the next reaction step.

To a solution of acid $18(0.32 \mathrm{~g}, 0.50 \mathrm{mmol})$ in $\mathrm{Et}_{2} \mathrm{O}(5.0 \mathrm{~mL})$ were added $\mathrm{Et}_{3} \mathrm{~N}(0.15 \mathrm{~g}, 1.5$ mmol) and $\mathrm{ClCO}_{2}{ }^{i} \mathrm{Bu}(0.14 \mathrm{~g}, 1.0 \mathrm{mmol})$ sequentially at $0{ }^{\circ} \mathrm{C}$. After $0.5 \mathrm{~h}$, a freshly prepared solution of $\mathrm{CH}_{2} \mathrm{~N}_{2}$ in $\mathrm{Et}_{2} \mathrm{O}(1.0 \mathrm{M}, 26 \mathrm{~mL})$ was added to the mixture at $0{ }^{\circ} \mathrm{C}$. The mixture was warmed to room temperature and stirred for $12 \mathrm{~h}$ before quenching with silica gel. The mixture was extracted with $\mathrm{Et}_{2} \mathrm{O}$ twice. The combined organic layers were dried over $\mathrm{MgSO}_{4}$, filtered, and concentrated under vacuum. The residue was purified by flash column chromatography on silica gel $\left(\right.$ EtOAc/hexanes $\left.=1: 4, \mathrm{R}_{\mathrm{f}}=0.46\right)$ to give compound $5(0.25 \mathrm{~g}, 77 \%)$ as a yellow foam that contains another unseparated diastereomer in a 1:10 ratio. 5: ${ }^{1} \mathrm{H}$ NMR $\left(400 \mathrm{MHz}, \mathrm{CDCl}_{3}\right) \delta 5.23(\mathrm{~s}$, $1 \mathrm{H}), 5.16(\mathrm{~d}, J=9.2 \mathrm{~Hz}, 1 \mathrm{H}), 4.33(\mathrm{~s}, 1 \mathrm{H}), 3.90(\mathrm{~s}, 1 \mathrm{H}), 3.68(\mathrm{~s}, 3 \mathrm{H}), 3.38(\mathrm{~d}, J=5.2 \mathrm{~Hz}, 1 \mathrm{H})$, 2.94 (brs, 1H), $2.74-2.64(\mathrm{~m}, 3 \mathrm{H}), 2.48-2.37(\mathrm{~m}, 1 \mathrm{H}), 2.25-2.12(\mathrm{~m}, 2 \mathrm{H}), 1.83(\mathrm{~d}, J=1.6 \mathrm{~Hz}$, 3H), $1.81-1.70(\mathrm{~m}, 1 \mathrm{H}), 1.58-1.55(\mathrm{~m}, 1 \mathrm{H}), 1.36(\mathrm{~d}, J=6.4 \mathrm{~Hz}, 3 \mathrm{H}), 0.89(\mathrm{~s}, 9 \mathrm{H}), 0.82(\mathrm{~s}, 9 \mathrm{H})$, 0.20 (s, 3H), 0.14 (s, 3H), 0.10 (s, 3H), 0.07 (s, 3H); ${ }^{13} \mathrm{C} \mathrm{NMR}\left(100 \mathrm{MHz}, \mathrm{CDCl}_{3}\right) \delta$ 209.7, 192.8, $175.3,82.4,80.4,72.7,66.8,62.8,54.8,51.9,47.9,46.8,44.4,42.9,42.8,34.0,31.4,25.9,25.6$, $23.3,18.2,17.9,15.0,3.5,-4.1,-4.9,-5.1,-5.2$; HRMS (ESI): $\mathrm{m} / z$ calcd for $\mathrm{C}_{32} \mathrm{H}_{53} \mathrm{ClN}_{2} \mathrm{O}_{6} \mathrm{NaSi}_{2}[\mathrm{M}+\mathrm{Na}]^{+}, 675.3023$, found: 675.3016; $[\alpha]_{\mathrm{D}}^{21.2}=-5.5\left(c 1.09, \mathrm{CHCl}_{3}\right)$. 

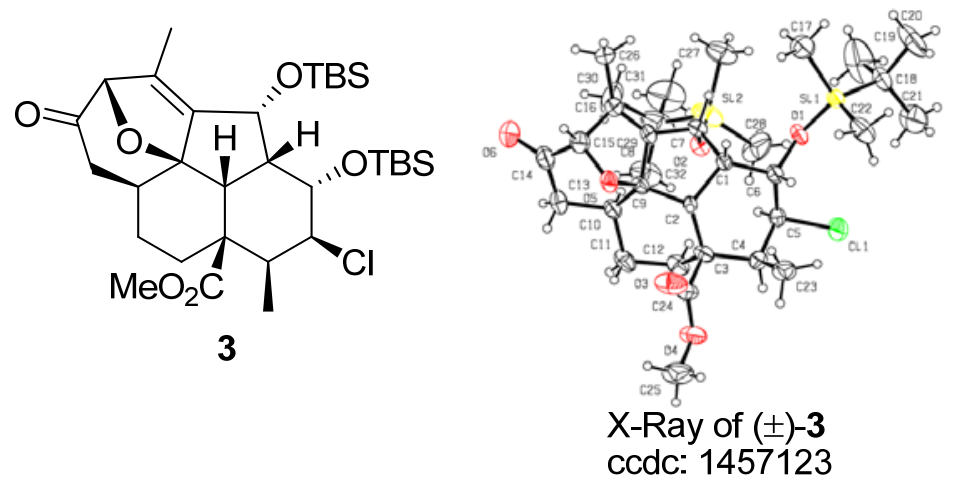

To a suspension of $\mathrm{Rh}_{2}(\mathrm{OAc})_{4}(0.016 \mathrm{~g}, 0.037 \mathrm{mmol})$ in refluxing toluene $(7.4 \mathrm{~mL})$ was added a solution of diazoketone $5(0.24 \mathrm{~g}, 0.37 \mathrm{mmol})$ in toluene $(7.4 \mathrm{~mL})$ over $0.5 \mathrm{~h}$ via a syringe pump. After additional $0.5 \mathrm{~h}$, the mixture was cooled to $\mathrm{rt}$ and the solvent was removed under vacuum. The residue was purified by flash column chromatography on silica gel (EtOAc/hexanes $=1: 4, \mathrm{R}_{\mathrm{f}}$ $=0.41)$ to afford $3(0.19 \mathrm{~g}, 81 \%)$ as a colorless solid. $3:{ }^{1} \mathrm{H}$ NMR $\left(400 \mathrm{MHz}, \mathrm{CDCl}_{3}\right) \delta 4.76(\mathrm{~d}, J=$ $3.6 \mathrm{~Hz}, 1 \mathrm{H}), 4.68(\mathrm{dd}, J=10.8,3.6 \mathrm{~Hz}, 1 \mathrm{H}), 4.62(\mathrm{~s}, 1 \mathrm{H}), 4.11-4.07(\mathrm{~m}, 1 \mathrm{H}), 3.75(\mathrm{~s}, 3 \mathrm{H}), 3.56$ $(\mathrm{d}, J=11.6 \mathrm{~Hz}, 1 \mathrm{H}), 2.89-2.83(\mathrm{~m}, 1 \mathrm{H}), 2.66-2.45(\mathrm{~m}, 4 \mathrm{H}), 2.11(\mathrm{td}, J=12.8,2.8 \mathrm{~Hz}, 1 \mathrm{H})$, $1.96(\mathrm{~d}, J=11.6 \mathrm{~Hz}, 1 \mathrm{H}), 1.79$ (s, 3H), 1.64 (brs, 1H), $1.12-1.04$ (m, 1H), 0.99 (d, $J=7.2 \mathrm{~Hz}$, 3H), 0.93 (s, 9H), 0.89 (s, 9H), 0.17 (s, 3H), 0.15 (s, 3H), 0.14 (s, 3H), 0.03 (s, 3H); ${ }^{13} \mathrm{C}$ NMR $\left(100 \mathrm{MHz}, \mathrm{CDCl}_{3}\right) \delta 205.1,176.2,149.5,127.3,95.2,93.8,69.8,67.8,63.4,52.7,52.4,50.1,44.6$, $44.3,39.8,39.3,30.7,27.9,26.3,25.7,18.7,18.1,11.3,11.2,-4.3,-4.4,-4.6,-4.9$; HRMS (ESI): $m / z$ calcd for $\mathrm{C}_{32} \mathrm{H}_{53} \mathrm{ClO}_{6} \mathrm{NaSi}_{2}[\mathrm{M}+\mathrm{Na}]^{+}, 647.2961$, found: $647.2953 ;[\alpha]_{\mathrm{D}}^{21.3}=-104.6(c$ $\left.1.08, \mathrm{CHCl}_{3}\right) ; \mathrm{mp}=52-54{ }^{\circ} \mathrm{C}$.

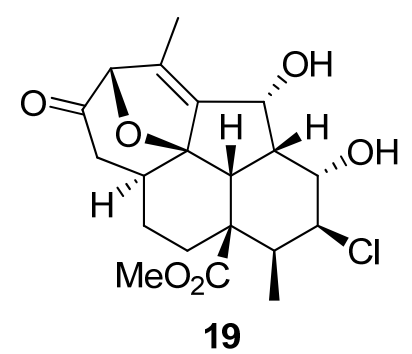

To a solution of $3(0.17 \mathrm{~g}, 0.27 \mathrm{mmol})$ in dry THF $(11 \mathrm{~mL})$ was added TBAF $(0.42 \mathrm{~g}, 1.4 \mathrm{mmol})$ at room temperature. The solution was quenched with $\mathrm{H}_{2} \mathrm{O}$ after the starting material disappeared as monitored by TLC. The mixture was extracted with DCM three times. The combined organic layers were dried over $\mathrm{Na}_{2} \mathrm{SO}_{4}$, filtered, and concentrated under vacuum. The residue was purified 
by flash column chromatography on silica gel $\left(\right.$ EtOAc/hexanes $\left.=1: 1, R_{\mathrm{f}}=0.31\right)$ to give compound 19 (91 mg, 85\%) as a colorless solid. 19: ${ }^{1} \mathrm{H}$ NMR $\left(400 \mathrm{MHz}, \mathrm{CDCl}_{3}\right) \delta 4.90(\mathrm{dd}, J=3.6,2.0 \mathrm{~Hz}$, 1H), 4.65 (s, 1H), 4.48 (dd, $J=11.2,3.6 \mathrm{~Hz}, 1 \mathrm{H}), 4.14$ (td, $J=10.4,2.8 \mathrm{~Hz}, 1 \mathrm{H}), 3.77$ (s, 3H), 3.60 (d, $J=12.8 \mathrm{~Hz}, 1 \mathrm{H}), 3.18-3.12(\mathrm{~m}, 1 \mathrm{H}), 3.00(\mathrm{~d}, J=2.4 \mathrm{~Hz}, 1 \mathrm{H}), 2.77(\mathrm{~d}, J=3.2 \mathrm{~Hz}, 1 \mathrm{H}), 2.62$ (d, $J=2.4 \mathrm{~Hz}, 1 \mathrm{H}), 2.60(\mathrm{~d}, J=2.0 \mathrm{~Hz}, 1 \mathrm{H}), 2.52-2.42(\mathrm{~m}, 2 \mathrm{H}), 2.05-2.02(\mathrm{~m}, 2 \mathrm{H}), 1.81(\mathrm{~s}$, $3 \mathrm{H}), 1.70-1.62(\mathrm{~m}, 1 \mathrm{H}), 1.15-1.00(\mathrm{~m}, 1 \mathrm{H}), 0.96(\mathrm{~d}, J=7.2 \mathrm{~Hz}, 3 \mathrm{H}) ;{ }^{13} \mathrm{C}$ NMR (100 MHz, $\left.\mathrm{CDCl}_{3}\right) \delta 204.9,175.7,147.3,130.2,95.1,94.1,70.4,67.8,66.6,52.6,50.7,49.0,44.6,43.5,39.9$, 39.1, 30.7, 28.3, 11.3, 10.7; HRMS (ESI): $m / z$ calcd for $\mathrm{C}_{20} \mathrm{H}_{25} \mathrm{ClO}_{6} \mathrm{Na}[\mathrm{M}+\mathrm{Na}]^{+}, 419.1232$, found: $419.1225 ;[\alpha]_{\mathrm{D}}^{21.6}=-192.7\left(\mathrm{c} 1.10, \mathrm{CHCl}_{3}\right) ; \mathrm{mp}=156-158^{\circ} \mathrm{C}$.

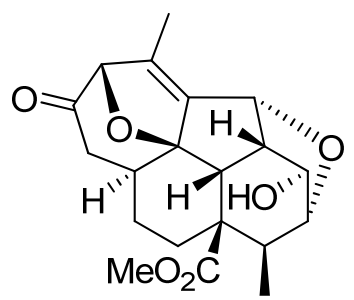

20

To a solution of $19(76 \mathrm{mg}, 0.19 \mathrm{mmol})$ in dry THF $(7.0 \mathrm{~mL})$ was added $\mathrm{NaH}(60 \%$ dispersion in mineral oil, $76 \mathrm{mg}, 1.9 \mathrm{mmol}$ ) at $0{ }^{\circ} \mathrm{C}$. The solution was allowed to warm to room temperature over $2 \mathrm{~h}$. After the starting material disappeared, the mixture was quenched with $\mathrm{H}_{2} \mathrm{O}$ and extracted with DCM. The combinedorganic layers were dried over $\mathrm{Na}_{2} \mathrm{SO}_{4}$, filtered, and concentrated under vacuum. The residue was purified by flash column chromatography on silica gel $\left(\right.$ EtOAc/hexanes $\left.=1: 1, \mathrm{R}_{\mathrm{f}}=0.25\right)$ to give compound $20(49 \mathrm{mg}, 71 \%)$ as a colorless solid. $\mathbf{2 0}$ : ${ }^{1} \mathrm{H}$ NMR $\left(400 \mathrm{MHz}, \mathrm{CDCl}_{3}\right) \delta 4.90(\mathrm{~d}, J=2.4 \mathrm{~Hz}, 1 \mathrm{H}), 4.54(\mathrm{~s}, 1 \mathrm{H}), 4.38(\mathrm{~d}, J=1.2 \mathrm{~Hz}, 1 \mathrm{H})$, $3.88(\mathrm{~s}, 1 \mathrm{H}), 3.69$ (s, 3H), 3.03 (q, $J=9.6 \mathrm{~Hz}, 2 \mathrm{H}), 2.91(\mathrm{dd}, J=18.8,8.4 \mathrm{~Hz}, 1 \mathrm{H}), 2.59-2.52(\mathrm{~m}$, 1H), $2.25(\mathrm{q}, J=7.6 \mathrm{~Hz}, 1 \mathrm{H}), 2.18(\mathrm{dd}, J=18.8,3.6 \mathrm{~Hz}, 1 \mathrm{H}), 2.10-2.02(\mathrm{~m}, 1 \mathrm{H}), 1.91-1.86(\mathrm{~m}$, 1H), $1.89(\mathrm{~s}, 3 \mathrm{H}), 1.84-1.70(\mathrm{~m}, 2 \mathrm{H}), 1.59$ (brs, $1 \mathrm{H}), 1.06(\mathrm{~d}, J=7.6 \mathrm{~Hz}, 3 \mathrm{H}) ;{ }^{13} \mathrm{C}$ NMR $(100$ $\left.\mathrm{MHz}, \mathrm{CDCl}_{3}\right) \delta 203.0,177.6,147.3,134.1,95.7,94.0,88.4,74.9,72.3,54.3,51.9,46.9,45.5,43.3$, 42.4, 31.3, 28.9, 26.0, 16.3, 11.5; HRMS (ESI): $\mathrm{m} / z$ calcd for $\mathrm{C}_{20} \mathrm{H}_{24} \mathrm{O}_{6} \mathrm{Na}[\mathrm{M}+\mathrm{Na}]^{+}, 383.1465$, found: $383.1457 ;[\alpha]_{\mathrm{D}}{ }^{18.5}=-309.7\left(c 1.03, \mathrm{CHCl}_{3}\right) ; \mathrm{mp}=253-255^{\circ} \mathrm{C}$. 


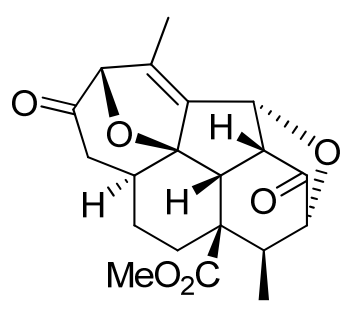

21

To a solution of 20 (35 mg, $0.098 \mathrm{mmol})$ in DCM $(4.0 \mathrm{~mL})$ were added $\mathrm{NaHCO}_{3}(0.16 \mathrm{~g}, 1.9$ mmol) and DMP (0.21 g, $0.49 \mathrm{mmol})$. The mixture was stirred for $24 \mathrm{~h}$ before quenching with $\mathrm{Na}_{2} \mathrm{~S}_{2} \mathrm{O}_{3}$ (aq) and $\mathrm{NaHCO}_{3}$ (aq). The solution was extracted with DCM three times. The combined organic layers were dried over $\mathrm{Na}_{2} \mathrm{SO}_{4}$, filtered, and concentrated under vacuum. The residue was purified by flash column chromatography on silica gel (EtOAc/hexanes $\left.=1: 1, \mathrm{R}_{\mathrm{f}}=0.65\right)$ to give compound 21 (23 mg, 66\%) as a colorless solid along with $9 \mathrm{mg}(25 \%)$ of recovered starting material. 21: ${ }^{1} \mathrm{H}$ NMR $\left(400 \mathrm{MHz}, \mathrm{CDCl}_{3}\right) \delta 4.77$ (d, $\left.J=2.4 \mathrm{~Hz}, 1 \mathrm{H}\right), 4.58(\mathrm{~s}, 1 \mathrm{H}), 3.65(\mathrm{~s}, 3 \mathrm{H})$, $3.60(\mathrm{~s}, 1 \mathrm{H}), 3.21(\mathrm{~d}, J=9.6 \mathrm{~Hz}, 1 \mathrm{H}), 3.05(\mathrm{dd}, J=9.2,2.4 \mathrm{~Hz}, 1 \mathrm{H}), 2.96(\mathrm{dd}, J=18.8,8.4 \mathrm{~Hz}$, 1H), $2.81-2.73(\mathrm{~m}, 1 \mathrm{H}), 2.57(\mathrm{q}, J=7.6 \mathrm{~Hz}, 1 \mathrm{H}), 2.28(\mathrm{dd}, J=18.4,3.6 \mathrm{~Hz}, 1 \mathrm{H}), 2.15-2.06(\mathrm{~m}$, 1H), $2.00-1.91(\mathrm{~m}, 2 \mathrm{H}), 1.89(\mathrm{~s}, 3 \mathrm{H}), 1.86-1.82(\mathrm{~m}, 1 \mathrm{H}), 0.98(\mathrm{~d}, J=7.6 \mathrm{~Hz}, 3 \mathrm{H}) ;{ }^{13} \mathrm{C} \mathrm{NMR}$ $\left(100 \mathrm{MHz}, \mathrm{CDCl}_{3}\right) \delta$ 208.0, 202.5, 175.0, 147.7, 135.8, 95.3, 94.4, 79.8, 72.4, 53.9, 53.3, 51.7, 48.6, 43.1, 42.4, 31.4, 27.5, 25.9, 15.0, 11.7; HRMS (ESI): $m / z$ calcd for $\mathrm{C}_{20} \mathrm{H}_{22} \mathrm{O}_{6} \mathrm{Na}[\mathrm{M}+\mathrm{Na}]^{+}$, 381.1309, found: $381.1302 ;[\alpha]_{\mathrm{D}}^{18.4}=-245.8\left(c 1.20, \mathrm{CHCl}_{3}\right) ; \mathrm{mp}=165-167^{\circ} \mathrm{C}$.

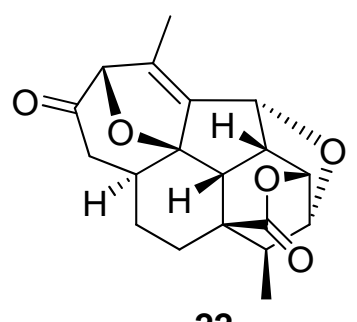

22

To a solution of $21(23.2 \mathrm{mg}, 0.0648 \mathrm{mmol})$ in $\mathrm{MeOH}(2.6 \mathrm{~mL})$ was added $\mathrm{NaBH}_{4}(10 \mathrm{mg}, 0.26$ mmol) at $0{ }^{\circ} \mathrm{C}$. After $5 \mathrm{~min}, \mathrm{~K}_{2} \mathrm{CO}_{3}(45 \mathrm{mg}, 0.32 \mathrm{mmol})$ was added to the mixture. The mixture was warmed to $60{ }^{\circ} \mathrm{C}$ and stirred for 20 min before quenching with $\mathrm{H}_{2} \mathrm{O}$. The mixture was extracted with DCM three times. The combined organic layers were dried over $\mathrm{Na}_{2} \mathrm{SO}_{4}$, filtered, and concentrated under vacuum. The crude product was used for the next reaction without further purification.

To a solution of the above crude product in $\mathrm{DCM}(2.6 \mathrm{~mL})$ were added $\mathrm{NaHCO}_{3}(54 \mathrm{mg}, 0.65$ 
mmol) and DMP (0.14 g, $0.32 \mathrm{mmol})$ at $\mathrm{rt}$. The mixture was stirred until the starting material disappeared as indicated by TLC. The mixture was quenched with $\mathrm{Na}_{2} \mathrm{~S}_{2} \mathrm{O}_{3}(\mathrm{aq})$ and $\mathrm{NaHCO}_{3}(\mathrm{aq})$ and extracted with DCM three times. The combined organic layers were dried over $\mathrm{Na}_{2} \mathrm{SO}_{4}$, filtered, and concentrated under vacuum. The residue was purified by flash column chromatography on silica gel $\left(\right.$ EtOAc/hexanes $\left.=1: 1, R_{\mathrm{f}}=0.44\right)$ to give compound $22(19.1 \mathrm{mg}, 90 \%$ over three steps) as a colorless foam. 22: ${ }^{1} \mathrm{H}$ NMR $\left(400 \mathrm{MHz}, \mathrm{CDCl}_{3}\right) \delta 5.13(\mathrm{t}, J=6.0 \mathrm{~Hz}, 1 \mathrm{H})$, $4.82(\mathrm{~d}, J=4.0 \mathrm{~Hz}, 1 \mathrm{H}), 4.50(\mathrm{~s}, 1 \mathrm{H}), 4.07(\mathrm{~d}, J=6.0 \mathrm{~Hz}, 1 \mathrm{H}), 3.28-3.23(\mathrm{~m}, 1 \mathrm{H}), 2.88-2.81$ (m, 1H), $2.52(\mathrm{~d}, J=10.0 \mathrm{~Hz}, 1 \mathrm{H}), 2.46-2.36(\mathrm{~m}, 2 \mathrm{H}), 2.27-2.17(\mathrm{~m}, 2 \mathrm{H}), 1.96-1.89(\mathrm{~m}, 2 \mathrm{H})$, $1.92(\mathrm{~s}, 3 \mathrm{H}), 1.85-1.76(\mathrm{~m}, 1 \mathrm{H}), 0.97(\mathrm{~d}, J=7.6 \mathrm{~Hz}, 3 \mathrm{H}) ;{ }^{13} \mathrm{C} \mathrm{NMR}\left(100 \mathrm{MHz}, \mathrm{CDCl}_{3}\right) \delta 203.4$, 174.0, 149.0, 142.3, 95.6, 92.8, 78.3, 77.8, 75.1, 47.8, 45.2, 43.7, 42.7, 41.3, 32.7, 26.5, 20.3, 15.0, 12.5; HRMS (ESI): $m / z$ calcd for $\mathrm{C}_{19} \mathrm{H}_{21} \mathrm{O}_{5}[\mathrm{M}+\mathrm{H}]^{+}, 329.1384$, found: $329.1390 ;[\alpha]_{\mathrm{D}}^{21.2}=-364.2$ (c $\left.1.85, \mathrm{CHCl}_{3}\right)$.
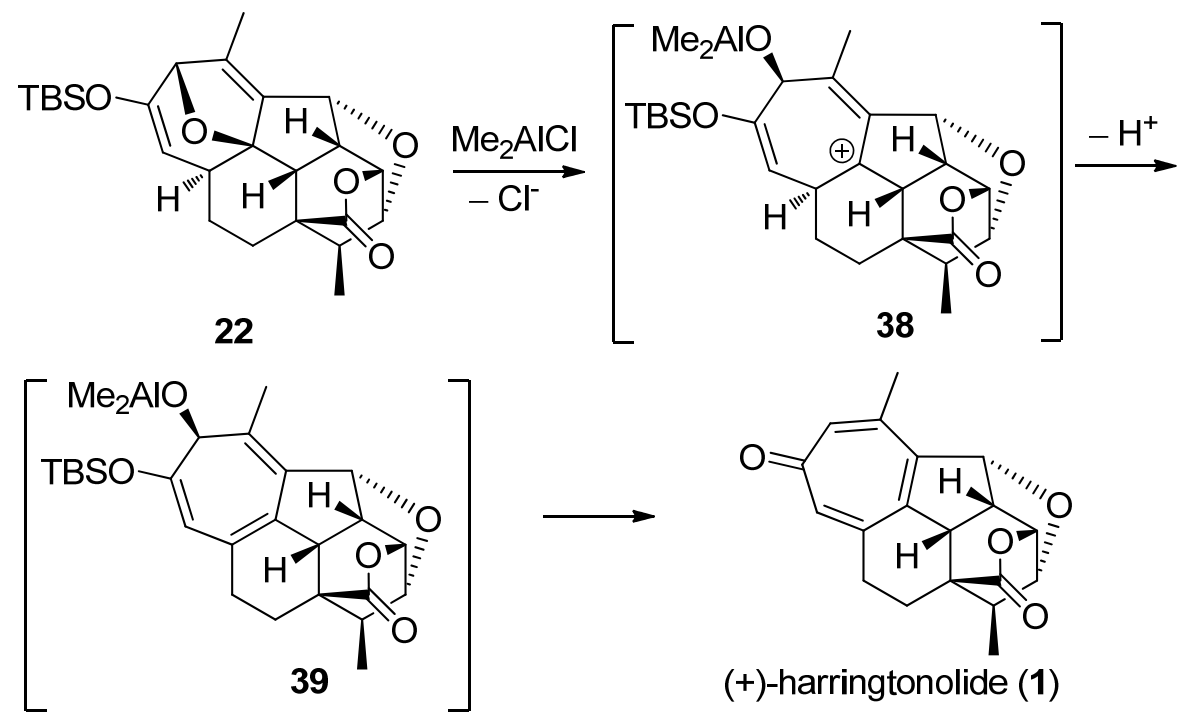

To a solution of $22(13.4 \mathrm{mg}, 0.0409 \mathrm{mmol})$ in DCM $(0.8 \mathrm{~mL})$ were added $\mathrm{Et}_{3} \mathrm{~N}(29 \mu \mathrm{L}, 0.21$ mmol) and TBSOTf (38 $\mu \mathrm{L}, 0.16 \mathrm{mmol})$ at room temperature. After the starting material disappeared, the mixture was quenched with $\mathrm{H}_{2} \mathrm{O}$ and extracted with DCM. The combined organic layers were dried over $\mathrm{Na}_{2} \mathrm{SO}_{4}$, filtered, and concentrated under vacuum. The crude product was used for the next reaction without further purification.

To a solution of the above crude product in $\mathrm{DCM}(3.0 \mathrm{~mL})$ was added $\mathrm{Me}_{2} \mathrm{AlCl}(0.9 \mathrm{M}$ in heptane, $2.3 \mathrm{~mL}, 2.1 \mathrm{mmol}$ ) at $-78{ }^{\circ} \mathrm{C}$. The mixture was allowed to warm to $40{ }^{\circ} \mathrm{C}$. After the starting material disappeared as indicated by TLC, the mixture was cooled to $-78{ }^{\circ} \mathrm{C}$ again and 
quenched with $\mathrm{MeOH}$ added dropwise at the temperature. The mixture was warmed to room temperature, diluted with $\mathrm{DCM}$, treated with $\mathrm{NH}_{4} \mathrm{Cl}$ (aq), and extracted with DCM three times. The combined organic layers were dried over $\mathrm{Na}_{2} \mathrm{SO}_{4}$, filtered, and concentrated under vacuum. The residue was purified by flash column chromatography on silica gel (EtOAc/hexanes $=1: 1, R_{f}$ $=0.15)$ to give natural product $(+)$-harringtonolide $(1,5.8 \mathrm{mg}, 46 \%$ over two steps $)$ as a light yellow solid. 1: ${ }^{1} \mathrm{H}$ NMR (400 MHz, $\left.\mathrm{CDCl}_{3}\right) \delta 6.97(\mathrm{~s}, 1 \mathrm{H}), 6.90(\mathrm{~s}, 1 \mathrm{H}), 5.36(\mathrm{t}, J=2.8 \mathrm{~Hz}, 1 \mathrm{H})$, $5.21(\mathrm{t}, J=5.2 \mathrm{~Hz}, 1 \mathrm{H}), 3.99(\mathrm{~d}, J=5.6 \mathrm{~Hz}, 1 \mathrm{H}), 3.42-3.36(\mathrm{~m}, 2 \mathrm{H}), 2.91-2.82(\mathrm{~m}, 2 \mathrm{H}), 2.66-$ $2.60(\mathrm{~m}, 1 \mathrm{H}), 2.37(\mathrm{~d}, J=0.8 \mathrm{~Hz}, 3 \mathrm{H}), 1.76(\mathrm{q}, J=7.6 \mathrm{~Hz}, 1 \mathrm{H}), 1.36-1.28(\mathrm{~m}, 1 \mathrm{H}), 0.90(\mathrm{~d}, J=$ 7.6 Hz, $3 \mathrm{H}) ;{ }^{13} \mathrm{C}$ NMR $\left(150 \mathrm{MHz}, \mathrm{CDCl}_{3}\right) \delta 186.5,173.5,145.8,145.6,145.0,143.6,141.6,139.3$, 86.0, 80.0, 79.7, 49.9, 45.8, 41.8, 40.0, 32.3, 23.9, 22.4, 14.7; HRMS (ESI): $\mathrm{m} / z$ calcd for $\mathrm{C}_{19} \mathrm{H}_{19} \mathrm{O}_{4}[\mathrm{M}+\mathrm{H}]^{+}, 311.1278$, found: $311.1283 ;[\alpha]_{\mathrm{D}}^{17.8}=+81.0\left(c\right.$ 1.16, $\left.\mathrm{CHCl}_{3}\right) ; \mathrm{mp}=255-258$ ${ }^{\circ} \mathrm{C}$.

\section{Construction of Oxapentacycle 19 from Compound 14'}




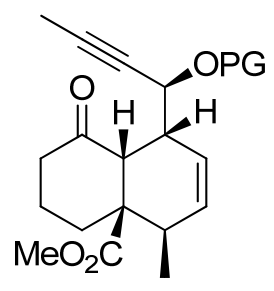

14'

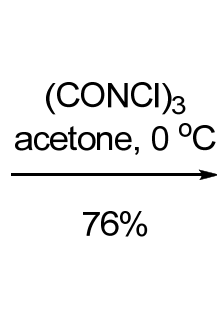

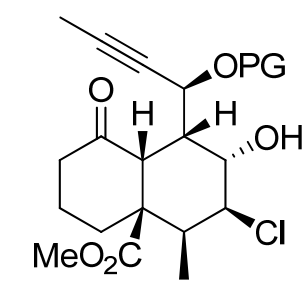

$15^{\prime}$

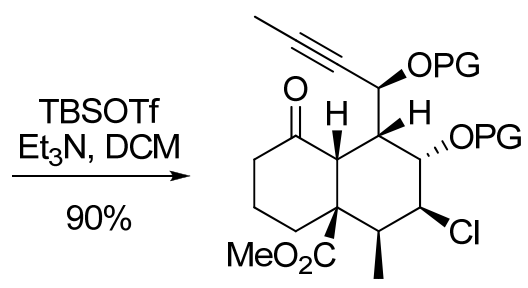

$16^{\prime}$

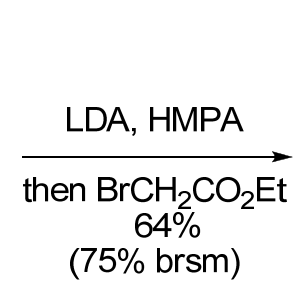

(75\% brsm)

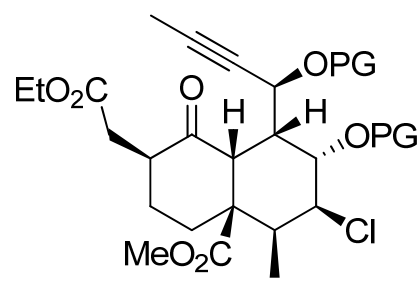

$17^{\prime}$

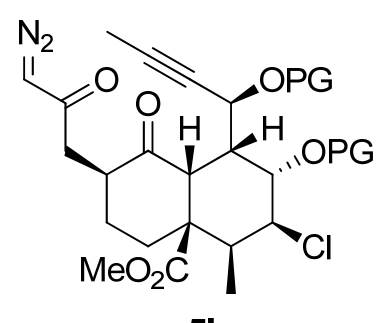

$5^{\prime}$

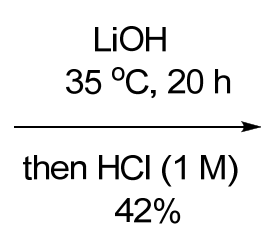

$42 \%$

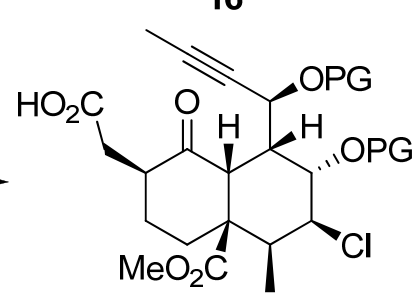

18'

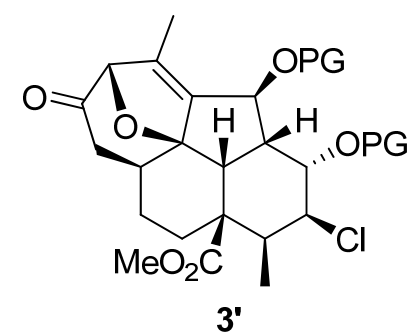

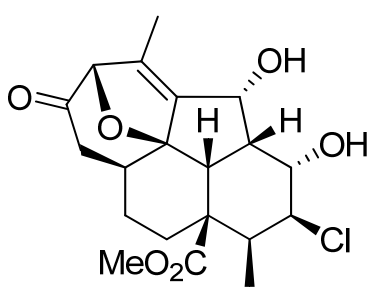

19

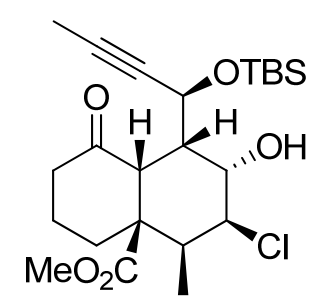

$15^{\prime}$

To a solution of 14' $(504 \mathrm{mg}, 1.25 \mathrm{mmol})$ in acetone $(5.6 \mathrm{~mL})$ was added $(\mathrm{CONCl})_{3}(0.23 \mathrm{~g}, 1.0$ mol) at $0{ }^{\circ} \mathrm{C}$. After being stirred for $0.5 \mathrm{~h}$, the mixture was quenched with $\mathrm{NaHSO}_{3}$ (aq) and extracted with EtOAc. The combined organic layers were dried over $\mathrm{Na}_{2} \mathrm{SO}_{4}$, filtered, and concentrated under vacuum. The residue was purified by flash column chromatography on silica gel (EtOAc/hexanes $\left.=1: 8, R_{\mathrm{f}}=0.53\right)$ to give compound 15' $(434 \mathrm{mg}, 76 \%)$ as a colorless foam that contains another unseparated diastereomer in a 1:16 ratio. 15': ${ }^{1} \mathrm{H} \mathrm{NMR}\left(400 \mathrm{MHz}, \mathrm{CDCl}_{3}\right) \delta$ 
$5.23(\mathrm{~d}, J=7.2 \mathrm{~Hz}, 1 \mathrm{H}), 4.85(\mathrm{dd}, J=10.4,2.4 \mathrm{~Hz}, 1 \mathrm{H}), 4.17-4.13(\mathrm{~m}, 1 \mathrm{H}), 4.04(\mathrm{t}, J=2.8 \mathrm{~Hz}$ $1 \mathrm{H}), 4.01(\mathrm{~d}, J=4.0 \mathrm{~Hz}, 1 \mathrm{H}), 3.74(\mathrm{~s}, 3 \mathrm{H}), 2.70(\mathrm{dt}, J=10.0,4.0 \mathrm{~Hz}, 1 \mathrm{H}), 2.52-2.44(\mathrm{~m}, 1 \mathrm{H})$, $2.38-2.32(\mathrm{~m}, 1 \mathrm{H}), 2.22-2.17(\mathrm{~m}, 1 \mathrm{H}), 2.11-1.93(\mathrm{~m}, 3 \mathrm{H}), 1.84(\mathrm{~d}, J=2.0 \mathrm{~Hz}, 3 \mathrm{H}), 1.80-$ $1.76(\mathrm{~m}, 1 \mathrm{H}), 1.30$ (d, $J=7.2 \mathrm{~Hz}, 3 \mathrm{H}), 0.91$ (s, 9H), $0.16(\mathrm{~s}, 3 \mathrm{H}), 0.15(\mathrm{~s}, 3 \mathrm{H}) ;{ }^{13} \mathrm{C}$ NMR $(100$ $\left.\mathrm{MHz}, \mathrm{CDCl}_{3}\right) \delta 218.4,175.1,82.4,79.0,69.4,67.0,61.2,53.0,52.2,52.2,41.8,40.8,34.8,34.5$, 25.8, 21.8, 18.2, 14.6, 3.6, - 4.5, - 5.2; HRMS (ESI): $\mathrm{m} / z$ calcd for $\mathrm{C}_{23} \mathrm{H}_{37} \mathrm{ClO}_{5} \mathrm{NaSi}[\mathrm{M}+\mathrm{Na}]^{+}$, 479.1991, found: $479.1997 ;[\alpha]_{\mathrm{D}}^{19.8}=-111.1\left(c 1.26, \mathrm{CHCl}_{3}\right)$.

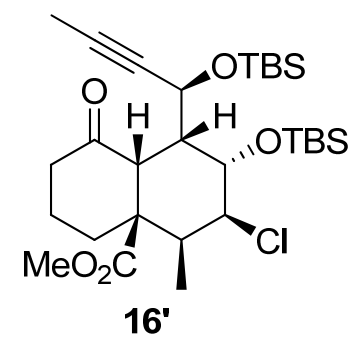

To a solution of 15' (1.01 g, $2.21 \mathrm{mmol})$ in DCM (11 mL) were added $\mathrm{Et}_{3} \mathrm{~N}(671 \mathrm{mg}, 6.63$ mmol) and TBSOTf $(1.46 \mathrm{~g}, 5.53 \mathrm{mmol})$ at $0{ }^{\circ} \mathrm{C}$. After being stirred for $3 \mathrm{~h}$, the mixture was quenched with $\mathrm{H}_{2} \mathrm{O}$ and extracted with EtOAc. The combined organic layers were dried over $\mathrm{Na}_{2} \mathrm{SO}_{4}$, filtered, and concentrated under vacuum. The residue was purified by flash column chromatography on silica gel $\left(\right.$ EtOAc/hexanes $\left.=1: 10, \mathrm{R}_{\mathrm{f}}=0.63\right)$ to give compound 16' $(1.1 \mathrm{~g}$, 90\%) a colorless foam. 16': ${ }^{1} \mathrm{H}$ NMR (400 MHz, $\left.\mathrm{CDCl}_{3}\right) \delta 5.31(\mathrm{dd}, J=10.0,2.4 \mathrm{~Hz}, 1 \mathrm{H}), 4.30$ $(\mathrm{dd}, J=2.8,1.6 \mathrm{~Hz}, 1 \mathrm{H}), 3.89$ (t, $J=2.8 \mathrm{~Hz}, 1 \mathrm{H}), 3.71(\mathrm{~s}, 3 \mathrm{H}), 3.36(\mathrm{~d}, J=4.8 \mathrm{~Hz}, 1 \mathrm{H}), 2.70-$ $2.61(\mathrm{~m}, 2 \mathrm{H}), 2.43-2.31(\mathrm{~m}, 2 \mathrm{H}), 2.24-2.15(\mathrm{~m}, 1 \mathrm{H}), 1.87-1.80(\mathrm{~m}, 2 \mathrm{H}), 1.82(\mathrm{~d}, J=2.0 \mathrm{~Hz}$, 3H), 1.67 (td, $J=13.6,5.2 \mathrm{~Hz}, 1 \mathrm{H}), 1.32$ (d, $J=6.8 \mathrm{~Hz}, 3 \mathrm{H}), 0.89$ (s, 9H), 0.84 (s, 9H), 0.21 (s, 3H), 0.19 (s, 3H), 0.13 (s, 3H), 0.04 (s, 3H); ${ }^{13} \mathrm{C} \mathrm{NMR}\left(100 \mathrm{MHz}, \mathrm{CDCl}_{3}\right) \delta$ 206.8, 176.0, 82.1, $79.4,70.5,67.1,62.9,51.9,51.4,49.3,43.8,39.5,33.1,32.5,26.1,25.7,18.4,18.2,17.9,14.5,3.8$, - 3.1, - 3.9, - 4.7, - 5.2; HRMS (ESI): $m / z$ calcd for $\mathrm{C}_{29} \mathrm{H}_{51} \mathrm{ClO}_{5} \mathrm{NaSi}_{2}[\mathrm{M}+\mathrm{Na}]^{+}, 593.2856$, found: $593.2846 ;[\alpha]_{\mathrm{D}}^{19.7}=-25.9\left(c 1.16, \mathrm{CHCl}_{3}\right)$.

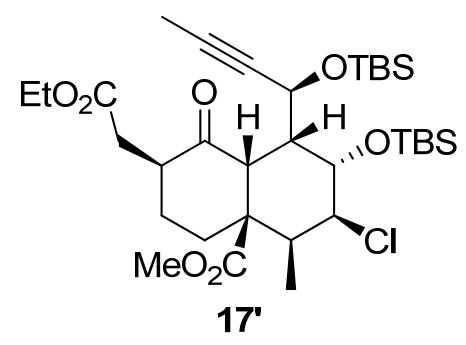

S18 
To a solution of freshly distilled diisopropylamine $(1.08 \mathrm{~g}, 10.7 \mathrm{mmol})$ in dry THF $(21 \mathrm{~mL})$ was added a solution of $n$-BuLi ( $2.5 \mathrm{M}$ in hexane, $3.4 \mathrm{~mL}, 8.5 \mathrm{mmol})$ dropwise at $-50{ }^{\circ} \mathrm{C}$. After $0.5 \mathrm{~h}$, the solution was cooled to $-78^{\circ} \mathrm{C}$ and then a solution of $\mathbf{1 6}^{\prime}(2.4 \mathrm{~g}, 4.3 \mathrm{mmol})$ in dry THF $(21 \mathrm{~mL})$ was added dropwise. After additional $1 \mathrm{~h}$, HMPA (2.29 g, $12.8 \mathrm{mmol})$ was added dropwise at the temperature. The resulting solution was stirred for $10 \mathrm{~min}$ and $\mathrm{BrCH}_{2} \mathrm{CO}_{2} \mathrm{Et}(1.06 \mathrm{~g}, 6.39 \mathrm{mmol})$ was added dropwise. The mixture was allowed to warm to $-40{ }^{\circ} \mathrm{C}$ and stirred for additional $1 \mathrm{~h}$ at the temperature. The mixture was quenched with $\mathrm{NH}_{4} \mathrm{Cl}$ (aq) at the temperature and extracted with EtOAc. The combined organic layers were washed with brine, dried over $\mathrm{MgSO}_{4}$, filtered, and concentrated under vacuum. The residue was purified by flash column chromatography on silica gel $\left(\right.$ EtOAc/hexanes $\left.=1: 10, \mathrm{R}_{\mathrm{f}}=0.62\right)$ to give compound 17' $(1.80 \mathrm{~g}, 64 \%)$ as a colorless oil along with $0.36 \mathrm{~g}(15 \%)$ of recovered starting material. 17': ${ }^{1} \mathrm{H}$ NMR (400 MHz, $\left.\mathrm{CDCl}_{3}\right) \delta 4.99$ $(\mathrm{dd}, J=10.0,2.0 \mathrm{~Hz}, 1 \mathrm{H}), 4.31(\mathrm{q}, J=1.6 \mathrm{~Hz}, 1 \mathrm{H}), 4.13-4.08(\mathrm{~m}, 2 \mathrm{H}), 3.91(\mathrm{t}, J=2.8 \mathrm{~Hz}, 1 \mathrm{H})$, $3.71(\mathrm{~s}, 3 \mathrm{H}), 3.47(\mathrm{~d}, J=4.8 \mathrm{~Hz}, 1 \mathrm{H}), 2.88-2.81(\mathrm{~m}, 1 \mathrm{H}), 2.74-2.66(\mathrm{~m}, 3 \mathrm{H}), 2.42(\mathrm{dd}, J=16.0$, $9.6 \mathrm{~Hz}, 1 \mathrm{H}), 2.21-2.11(\mathrm{~m}, 1 \mathrm{H}), 2.09-2.03(\mathrm{~m}, 1 \mathrm{H}), 1.92-1.87(\mathrm{~m}, 1 \mathrm{H}), 1.85(\mathrm{~d}, J=2.0 \mathrm{~Hz}$, 3H), $1.61-1.55(\mathrm{~m}, 1 \mathrm{H}), 1.35(\mathrm{~d}, J=7.2 \mathrm{~Hz}, 3 \mathrm{H}), 1.24$ (t, $J=7.2 \mathrm{~Hz}, 3 \mathrm{H}), 0.88$ (s, 9H), 0.83 (s, 9H), $0.21(\mathrm{~s}, 3 \mathrm{H}), 0.18(\mathrm{~s}, 3 \mathrm{H}), 0.10(\mathrm{~s}, 3 \mathrm{H}), 0.05(\mathrm{~s}, 3 \mathrm{H}) ;{ }^{13} \mathrm{C} \mathrm{NMR}\left(100 \mathrm{MHz}, \mathrm{CDCl}_{3}\right) \delta 209.3$, 175.5, 172.0, 82.5, 79.6, 70.6, 66.5, 62.6, 60.5, 51.9, 49.5, 48.2, 44.0, 42.5, 36.8, 34.2, 31.7, 26.1, 25.6, 23.4, 18.2, 17.9, 14.9, 14.2, 3.8, - 3.1, - 3.8, - 4.8, - 5.1; HRMS (ESI): $m / z$ calcd for $\mathrm{C}_{33} \mathrm{H}_{57} \mathrm{ClO}_{7} \mathrm{NaSi}_{2}[\mathrm{M}+\mathrm{Na}]^{+}, 679.3224$, found: $679.3232 ;[\alpha]_{\mathrm{D}}^{19.7}=-61.5\left(c 0.95, \mathrm{CHCl}_{3}\right)$.

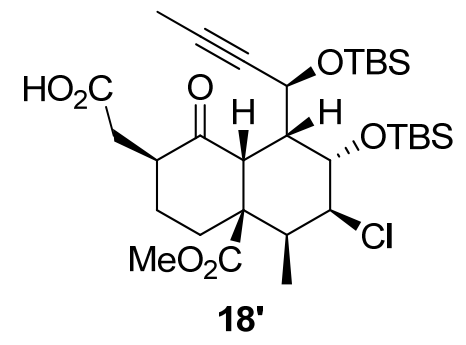

To a solution of the crude 17' $(1.7 \mathrm{~g}, 2.6 \mathrm{mmol})$ in $\mathrm{THF} / \mathrm{H}_{2} \mathrm{O}(4: 1,26 \mathrm{~mL})$ was added $\mathrm{LiOH}$ (551 mg, $13.1 \mathrm{mmol}$ ). After the mixture was stirred vigorously for $20 \mathrm{~h}$ at $35^{\circ} \mathrm{C}$, the mixture was diluted with DCM and washed with $1.0 \mathrm{M} \mathrm{HCl}(\mathrm{aq})$. The organic layer was dried over $\mathrm{MgSO}_{4}$, filtered, and concentrated under vacuum. The residue was purified by flash column chromatography on silica gel (EtOAc/hexanes $\left.=1: 4, \mathrm{R}_{\mathrm{f}}=0.21\right)$ to give compound 18' $(0.65 \mathrm{~g}$, 
$42 \%)$ as a colorless foam that contains another unseparated diastereomer in a 1:14 ratio. 18': ${ }^{1} \mathrm{H}$ NMR (400 MHz, $\left.\mathrm{CDCl}_{3}\right) \delta 4.99-4.96(\mathrm{~m}, 1 \mathrm{H}), 4.32(\mathrm{~s}, 1 \mathrm{H}), 3.91(\mathrm{~s}, 1 \mathrm{H}), 3.72(\mathrm{~s}, 3 \mathrm{H}), 3.47(\mathrm{~d}, J$ $=5.2 \mathrm{~Hz}, 1 \mathrm{H}), 2.86-2.84(\mathrm{~m}, 1 \mathrm{H}), 2.80-2.69(\mathrm{~m}, 3 \mathrm{H}), 2.46(\mathrm{dd}, J=16.4,8.8 \mathrm{~Hz}, 1 \mathrm{H}), 2.25-$ $2.16(\mathrm{~m}, 1 \mathrm{H}), 2.11-2.05(\mathrm{~m}, 1 \mathrm{H}), 1.91-1.81(\mathrm{~m}, 1 \mathrm{H}), 1.86(\mathrm{~d}, J=1.6 \mathrm{~Hz}, 3 \mathrm{H}), 1.63-1.58(\mathrm{~m}$, 1H), 1.36 (d, $J=7.2 \mathrm{~Hz}, 3 \mathrm{H}), 0.89$ (s, 9H), 0.83 (s, 9H), 0.21 (s, 3H), 0.18 (s, 3H), 0.10 (s, 3H), 0.05 (s, 3H); ${ }^{13} \mathrm{C}$ NMR $\left(100 \mathrm{MHz}, \mathrm{CDCl}_{3}\right) \delta 209.4,177.5,175.5,82.6,79.5,70.6,66.4,62.6,52.0$, $49.5,48.1,44.1,42.2,36.4,34.4,31.7,26.1,25.6,23.5,18.2,17.9,15.0,3.8,-3.1,-3.8,-4.9,-$ 5.1; HRMS (ESI): $m / z$ calcd for $\mathrm{C}_{31} \mathrm{H}_{53} \mathrm{ClO}_{7} \mathrm{NaSi}_{2}[\mathrm{M}+\mathrm{Na}]^{+}, 651.2911$, found: $651.2919 ;[\alpha]_{\mathrm{D}}^{21.8}=$ - $54.7\left(c 1.17, \mathrm{CHCl}_{3}\right)$.

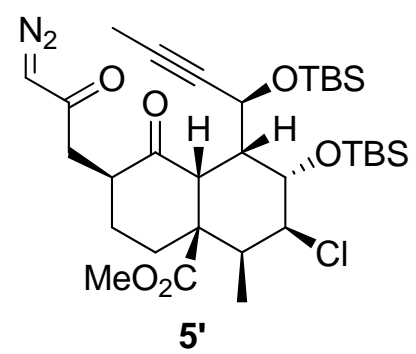

Preparation of $\mathrm{CH}_{2} \mathrm{~N}_{2}$ : under continuously shaking, $\mathrm{CH}_{3} \mathrm{~N}(\mathrm{NO}) \mathrm{CONH}_{2}(3.4 \mathrm{~g}, 33 \mathrm{mmol})$ was added to a mixture of $\mathrm{KOH}\left(40 \%\right.$ in $\left.\mathrm{H}_{2} \mathrm{O}, 11.2 \mathrm{~mL}\right)$ and $\mathrm{Et}_{2} \mathrm{O}(34 \mathrm{~mL})$ at $0{ }^{\circ} \mathrm{C}$. Decantation of the light yellow solution gave the solution of $\mathrm{CH}_{2} \mathrm{~N}_{2}\left(1.0 \mathrm{M}\right.$ in $\left.\mathrm{Et}_{2} \mathrm{O}\right)$. The solution was dried over dusty $\mathrm{KOH}$ and used for the next reaction step.

To a solution of acid 18' $(0.41 \mathrm{~g}, 0.65 \mathrm{mmol})$ in $\mathrm{Et}_{2} \mathrm{O}(6.5 \mathrm{~mL})$ were added $\mathrm{Et}_{3} \mathrm{~N}(0.20 \mathrm{~g}, 2.0$ $\mathrm{mmol})$ and $\mathrm{ClCO}_{2}{ }^{i} \mathrm{Bu}(0.18 \mathrm{~g}, 1.3 \mathrm{mmol})$ sequentially at $0{ }^{\circ} \mathrm{C}$. After $0.5 \mathrm{~h}$, a freshly prepared solution of $\mathrm{CH}_{2} \mathrm{~N}_{2}\left(34 \mathrm{~mL}, 1.0 \mathrm{M}\right.$ in $\left.\mathrm{Et}_{2} \mathrm{O}\right)$ was added to the mixture at $0{ }^{\circ} \mathrm{C}$. The mixture was warmed to room temperature and stirred for $12 \mathrm{~h}$ before quenching with silica gel. The mixture was extracted with $\mathrm{Et}_{2} \mathrm{O}$ twice. The combined organic layers were dried over $\mathrm{MgSO}_{4}$, filtered, and concentrated under vacuum. The residue was purified by flash column chromatography on silica gel $\left(\right.$ EtOAc/hexanes $\left.=1: 4, R_{\mathrm{f}}=0.23\right)$ to give compound $\mathbf{5}^{\prime}(0.33 \mathrm{~g}, 77 \%)$ as a yellow oil that contains another unseparated diastereomer in a 1:14 ratio. 5': ${ }^{1} \mathrm{H}$ NMR $\left(400 \mathrm{MHz}, \mathrm{CDCl}_{3}\right) \delta 5.24$ (s, 1H), 4.99 (dd, $J=10.0,2.0 \mathrm{~Hz}, 1 \mathrm{H}), 4.31$ (d, $J=1.6 \mathrm{~Hz}, 1 \mathrm{H}), 3.90$ (t, $J=2.4 \mathrm{~Hz}, 1 \mathrm{H}), 3.70$ (s, 3H), 3.46 (d, $J=5.2 \mathrm{~Hz}, 1 \mathrm{H}), 2.88$ (brs, 1H), $2.74-2.67$ (m, 3H), 2.41 (brs, 1H), $2.18-2.12$ (m, 1H), $2.10-2.04(\mathrm{~m}, 1 \mathrm{H}), 1.91-1.81(\mathrm{~m}, 1 \mathrm{H}), 1.85(\mathrm{~d}, J=2.0 \mathrm{~Hz}, 3 \mathrm{H}), 1.60-1.55(\mathrm{~m}, 1 \mathrm{H}), 1.34$ 
(d, $J=7.2 \mathrm{~Hz}, 3 \mathrm{H}), 0.88(\mathrm{~s}, 9 \mathrm{H}), 0.82(\mathrm{~s}, 9 \mathrm{H}), 0.20(\mathrm{~s}, 3 \mathrm{H}), 0.17$ (s, 3H), 0.09 (s, 3H), $0.06(\mathrm{~s}, 3 \mathrm{H})$; ${ }^{13} \mathrm{C}$ NMR $\left(100 \mathrm{MHz}, \mathrm{CDCl}_{3}\right) \delta 209.7,192.7,175.5,82.4,79.6,70.6,66.5,62.6,54.7,51.9,49.5$, $48.1,44.1,42.8,42.8,34.2,31.6,26.1,25.6,23.5,18.2,17.9,14.9,3.8,-3.1,-3.8,-4.8,-5.2$; HRMS (ESI): $m / z$ calcd for $\mathrm{C}_{32} \mathrm{H}_{53} \mathrm{ClN}_{2} \mathrm{O}_{6} \mathrm{NaSi}_{2}[\mathrm{M}+\mathrm{Na}]^{+}, 675.3023$, found: $675.3032 ;[\alpha]_{\mathrm{D}}^{21.2}=$ $-47.6\left(c 1.37, \mathrm{CHCl}_{3}\right)$.

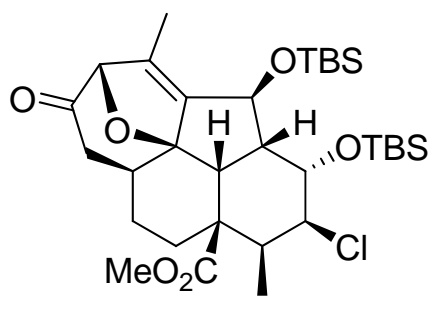

3'

To a suspension of $\mathrm{Rh}_{2}(\mathrm{OAc})_{4}(0.022 \mathrm{~g}, 0.050 \mathrm{mmol})$ in refluxing toluene $(10 \mathrm{~mL})$ was added a solution of diazoketone $5^{\prime}(0.33 \mathrm{~g}, 0.50 \mathrm{mmol})$ in toluene $(10 \mathrm{~mL})$ over $0.5 \mathrm{~h}$ via a syringe pump. After additional $0.5 \mathrm{~h}$, the solution was cooled to $\mathrm{rt}$ and the solvent was removed under vacuum. The residue was purified by flash column chromatography on silica gel (EtOAc/hexanes $=1: 4, R_{\mathrm{f}}$ $=0.57)$ to afford compound 3' $(0.20 \mathrm{~g}, 62 \%)$ as a colorless solid. 3': ${ }^{1} \mathrm{H}$ NMR (400 MHz, $\left.\mathrm{CDCl}_{3}\right)$ $\delta 4.77(\mathrm{~s}, 1 \mathrm{H}), 4.57$ (s, 1H), $4.25(\mathrm{dd}, J=4.8,1.6 \mathrm{~Hz}, 1 \mathrm{H}), 4.19$ (d, $J=4.8 \mathrm{~Hz}, 1 \mathrm{H}), 3.69$ (s, 3H), 3.47 (d, $J=12.0 \mathrm{~Hz}, 1 \mathrm{H}), 2.93(\mathrm{dq}, J=12.0,2.4 \mathrm{~Hz}, 1 \mathrm{H}), 2.83(\mathrm{dd}, J=18.4,8.4 \mathrm{~Hz}, 1 \mathrm{H}), 2.53-$ $2.50(\mathrm{~m}, 2 \mathrm{H}), 2.28-2.21(\mathrm{~m}, 1 \mathrm{H}), 2.12(\mathrm{~d}, J=18.8 \mathrm{~Hz}, 1 \mathrm{H}), 1.87-1.83(\mathrm{~m}, 1 \mathrm{H}), 1.80(\mathrm{~d}, J=1.2$ $\mathrm{Hz}, 3 \mathrm{H}), 1.73-1.67$ (m, 1H), $1.51-1.42(\mathrm{~m}, 1 \mathrm{H}), 1.08$ (d, J= $7.2 \mathrm{~Hz}, 3 \mathrm{H}), 0.90$ (s, 9H), 0.87 (s, 9H), 0.19 (s, 3H), 0.18 (s, 3H), $0.12(\mathrm{~s}, 3 \mathrm{H}), 0.11(\mathrm{~s}, 3 \mathrm{H}) ;{ }^{13} \mathrm{C} \mathrm{NMR}\left(100 \mathrm{MHz}, \mathrm{CDCl}_{3}\right) \delta 203.8$, $175.8,149.7,135.1,96.2,96.1,75.0,68.1,65.0,52.6,52.1,46.6,41.3,38.2,35.1,30.9,28.0,27.3$, 25.7, 25.6, 18.0, 17.9, 14.2, 11.5, - 3.6, - 4.1, - 4.7, - 4.8; HRMS (ESI): $\mathrm{m} / \mathrm{z}$ calcd for $\mathrm{C}_{32} \mathrm{H}_{53} \mathrm{ClO}_{6} \mathrm{NaSi}_{2}[\mathrm{M}+\mathrm{Na}]^{+}, 647.2961$, found: 647.2973; $[\alpha]_{\mathrm{D}}^{22.0}=-213.5\left(c 1.26, \mathrm{CHCl}_{3}\right) ; \mathrm{mp}=$ $36-37^{\circ} \mathrm{C}$.

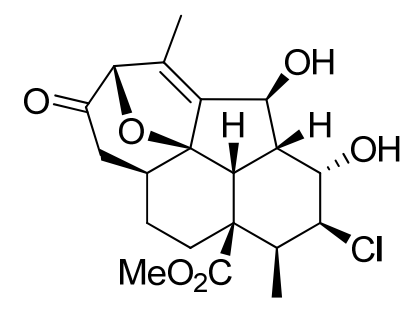

19'

To a solution of 3' $(0.18 \mathrm{~g}, 0.29 \mathrm{mmol})$ in dry THF $(12 \mathrm{~mL})$ was added TBAF $(0.28 \mathrm{~g}, 0.87$ 
mmol) at room temperature. The solution was quenched with $\mathrm{H}_{2} \mathrm{O}$ after the starting material disappeared as indicated by TLC. The mixture was extracted with DCM three times. The combined organic layers were dried over $\mathrm{Na}_{2} \mathrm{SO}_{4}$, filtered, and concentrated under vacuum. The residue was purified by flash column chromatography on silica gel (EtOAc/hexanes $=1: 1, R_{\mathrm{f}}=$ 0.33 ) to give compound 19' (92 mg, 79\%) as a colorless solid. 19': ${ }^{1} \mathrm{H} \mathrm{NMR}\left(400 \mathrm{MHz}, \mathrm{CDCl}_{3}\right) \delta$ 4.78 (d, $J=10.8 \mathrm{~Hz}, 1 \mathrm{H}), 4.63$ (s, 1H), 4.34 (dd, $J=10.8,3.2 \mathrm{~Hz}, 1 \mathrm{H}), 4.19$ (t, $J=10.0 \mathrm{~Hz}, 1 \mathrm{H})$, $3.77(\mathrm{~s}, 3 \mathrm{H}), 3.67(\mathrm{~d}, J=13.2 \mathrm{~Hz}, 1 \mathrm{H}), 3.63(\mathrm{~s}, 1 \mathrm{H}), 3.10-3.01(\mathrm{~m}, 1 \mathrm{H}), 2.76(\mathrm{~s}, 1 \mathrm{H}), 2.64-2.52$ (m, 2H), $2.45-2.39(\mathrm{~m}, 1 \mathrm{H}), 2.15-2.11(\mathrm{~m}, 1 \mathrm{H}), 1.87(\mathrm{~d}, J=1.6 \mathrm{~Hz}, 3 \mathrm{H}), 1.80-1.72(\mathrm{~m}, 1 \mathrm{H})$, $1.68-1.54(\mathrm{~m}, 2 \mathrm{H}), 1.07$ (q, $J=12.8 \mathrm{~Hz}, 1 \mathrm{H}), 0.95$ (d, $J=7.2 \mathrm{~Hz}, 3 \mathrm{H}) ;{ }^{13} \mathrm{C}$ NMR (100 MHz, $\left.\mathrm{CDCl}_{3}\right) \delta 204.2,175.1,146.7,127.4,94.4,90.9,70.4,70.2,65.2,52.7,50.5,48.3,43.5,43.4,42.1$, 38.0, 32.1, 27.8, 10.5, 9.8; HRMS (ESI): $m / z$ calcd for $\mathrm{C}_{20} \mathrm{H}_{25} \mathrm{ClO}_{6} \mathrm{Na}[\mathrm{M}+\mathrm{Na}]^{+}$, 419.1232, found: $419.1238 ;[\alpha]_{\mathrm{D}}^{22.2}=-225.7\left(\mathrm{c} 1.13, \mathrm{CHCl}_{3}\right) ; \mathrm{mp}=78-81^{\circ} \mathrm{C}$.

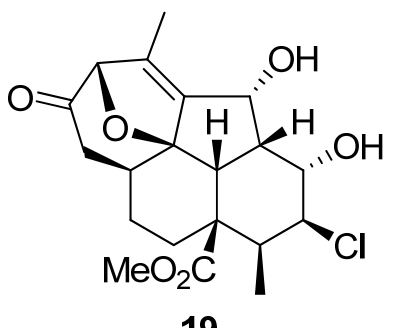

19

To a solution of 19' $(78 \mathrm{mg}, 0.20 \mathrm{mmol})$ in dry THF $(4.0 \mathrm{~mL})$ were added $\mathrm{Ph}_{3} \mathrm{P}(0.16 \mathrm{~g}, 0.59$ mmol), 4- $\mathrm{NO}_{2}-\mathrm{C}_{6} \mathrm{H}_{4} \mathrm{CO}_{2} \mathrm{H}(99 \mathrm{mg}, 0.59 \mathrm{mmol})$ and DEAD (0.10 g, $\left.0.59 \mathrm{mmol}\right)$ sequentially at room temperature. The solution was quenched with $\mathrm{H}_{2} \mathrm{O}$ after the starting material disappeared as indicated by TLC. The mixture was extracted with DCM three times. The combined organic layers were dried over $\mathrm{Na}_{2} \mathrm{SO}_{4}$, filtered, and concentrated under vacuum. The residue was purified by flash column chromatography on silica gel (EtOAc/hexanes $\left.=1: 1, \mathrm{R}_{\mathrm{f}}=0.40\right)$ to give a crude product used for the next step.

To a solution of the above crude product in $\mathrm{MeOH}(2.0 \mathrm{~mL})$ was added $\mathrm{K}_{2} \mathrm{CO}_{3}(54 \mathrm{mg}, 0.59$ mmol). The solution was quenched with $\mathrm{H}_{2} \mathrm{O}$ after the starting material disappeared as indicated by TLC. The mixture was extracted with DCM three times. The combined organic layers were dried over $\mathrm{Na}_{2} \mathrm{SO}_{4}$, filtered, and concentrated under vacuum. The residue was purified by flash column chromatography on silica gel to give 19 (25 mg, $33 \%$ over two steps) as a colorless solid. 
The ${ }^{1} \mathrm{H}$ NMR and ${ }^{13} \mathrm{C}$ NMR was in agreement with those measured above.

\section{Some Important Intermediates (A-E)}

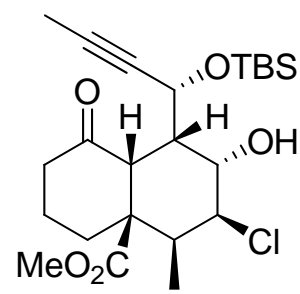

15

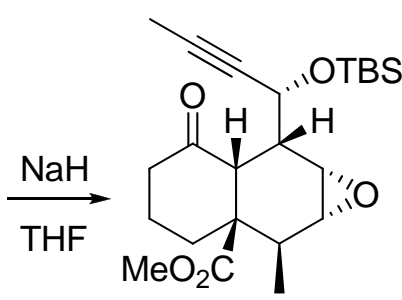

24

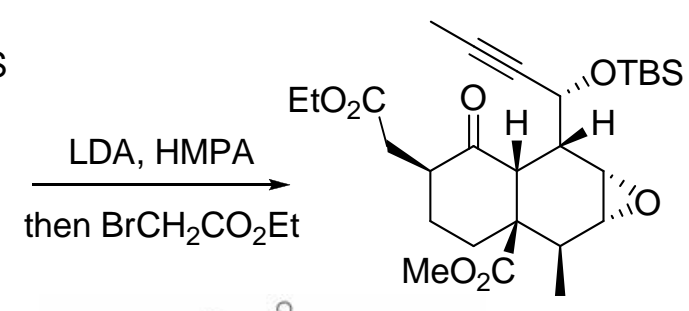

25

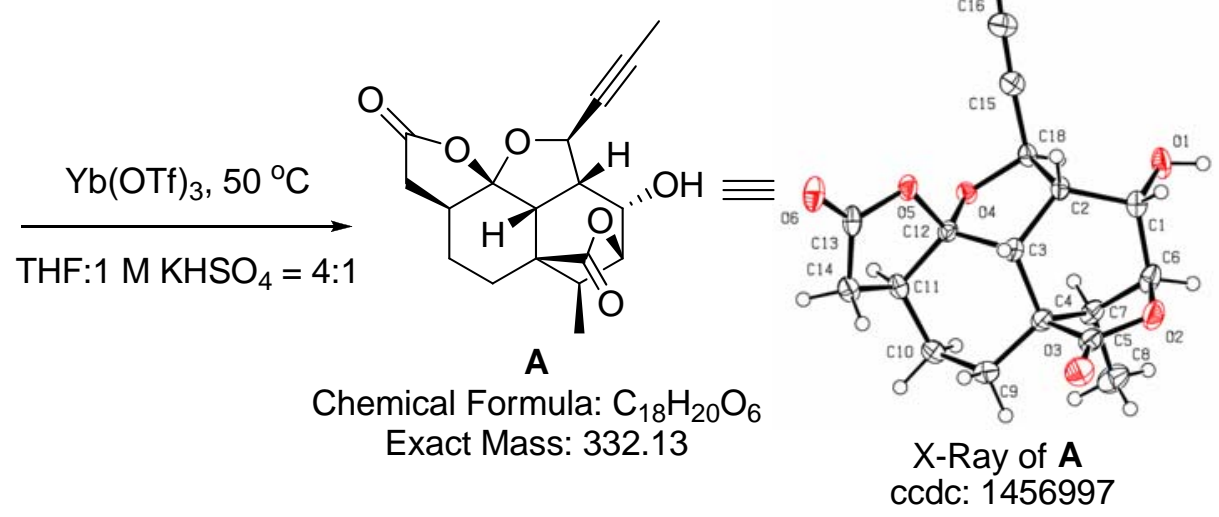

A: ${ }^{1} \mathrm{H}$ NMR $\left(400 \mathrm{MHz}, \mathrm{CDCl}_{3}\right) \delta 5.28-5.24(\mathrm{~m}, 1 \mathrm{H}), 4.36-4.30(\mathrm{~m}, 1 \mathrm{H}), 4.28(\mathrm{~s}, 1 \mathrm{H}), 3.42(\mathrm{q}$, $J=8.4 \mathrm{~Hz}, 1 \mathrm{H}), 2.93-2.86(\mathrm{~m}, 1 \mathrm{H}), 2.71-2.55(\mathrm{~m}, 3 \mathrm{H}), 2.50-2.41(\mathrm{~m}, 2 \mathrm{H}), 1.93-1.85(\mathrm{~m}$, 4H), $1.80-1.73(\mathrm{~m}, 1 \mathrm{H}), 1.71-1.58(\mathrm{~m}, 2 \mathrm{H}), 1.06(\mathrm{~d}, J=6.8 \mathrm{~Hz}, 3 \mathrm{H}) ;{ }^{13} \mathrm{C}$ NMR $(100 \mathrm{MHz}$, $\left.\mathrm{CDCl}_{3}\right) \delta 178.9,172.5,116.7,85.2,83.0,78.8,72.4,66.8,46.9,45.7,45.6,37.1,34.2,33.1,19.3$, 19.2, 13.3, 3.9. 


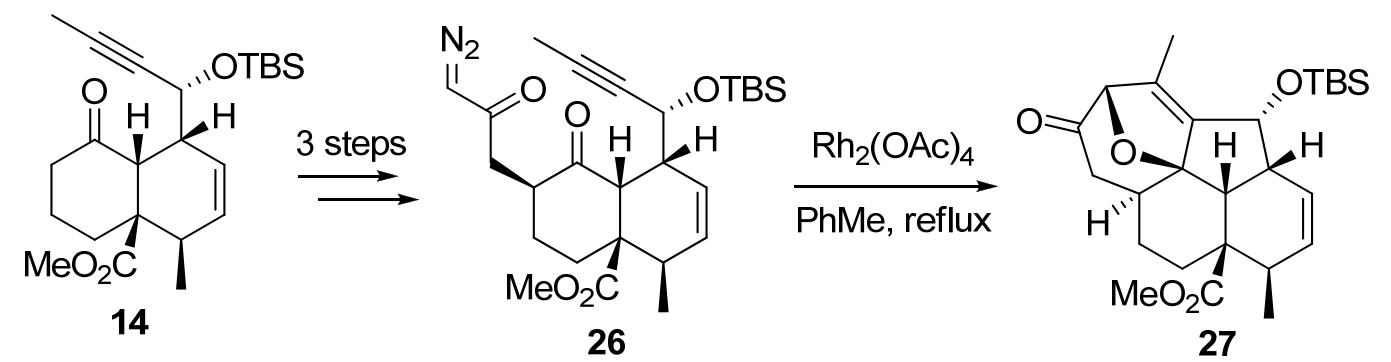

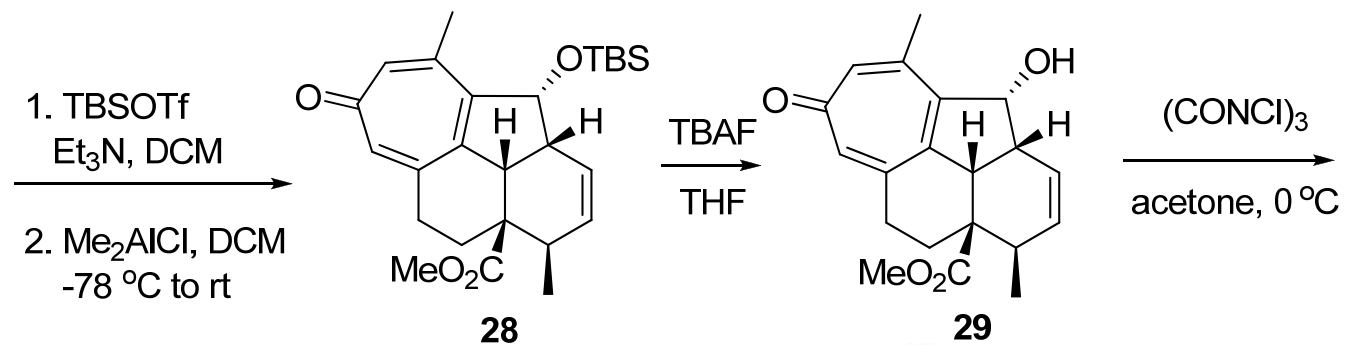

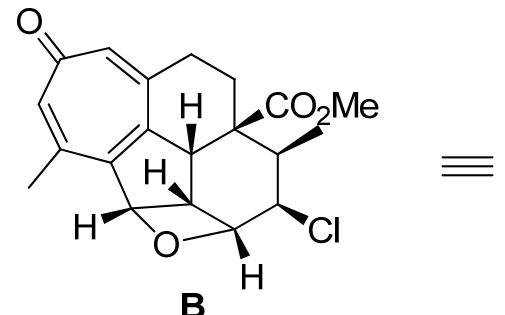

Chemical Formula: $\mathrm{C}_{20} \mathrm{H}_{21} \mathrm{ClO}_{4}$ Exact Mass: 360.11

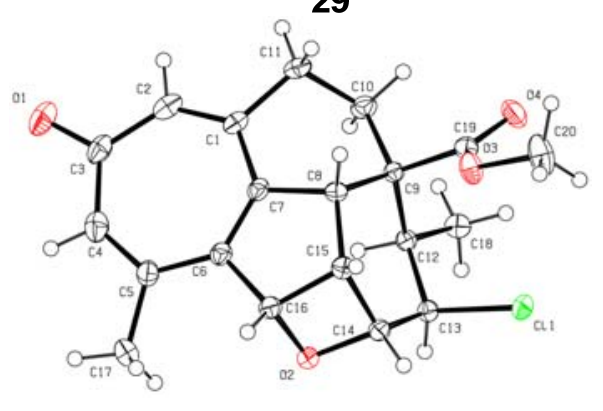

X-Ray of B
ccdc: 1457121

B: ${ }^{1} \mathrm{H}$ NMR $\left(400 \mathrm{MHz}, \mathrm{CDCl}_{3}\right) \delta 6.94(\mathrm{~s}, 1 \mathrm{H}), 6.89(\mathrm{~s}, 1 \mathrm{H}), 5.95(\mathrm{~d}, J=5.6 \mathrm{~Hz}, 1 \mathrm{H}), 5.20(\mathrm{dd}, J=$ 7.2, $2.8 \mathrm{~Hz}, 1 \mathrm{H}), 3.96$ (t, $J=2.8 \mathrm{~Hz}, 1 \mathrm{H}), 3.90(\mathrm{~d}, J=9.6 \mathrm{~Hz}, 1 \mathrm{H}), 3.75(\mathrm{~s}, 3 \mathrm{H}), 3.69-3.63(\mathrm{~m}$, 1H), $2.67-2.50(\mathrm{~m}, 3 \mathrm{H}), 2.27(\mathrm{~s}, 3 \mathrm{H}), 1.69-1.62(\mathrm{~m}, 2 \mathrm{H}), 1.26(\mathrm{~d}, J=6.8 \mathrm{~Hz}, 3 \mathrm{H})$.

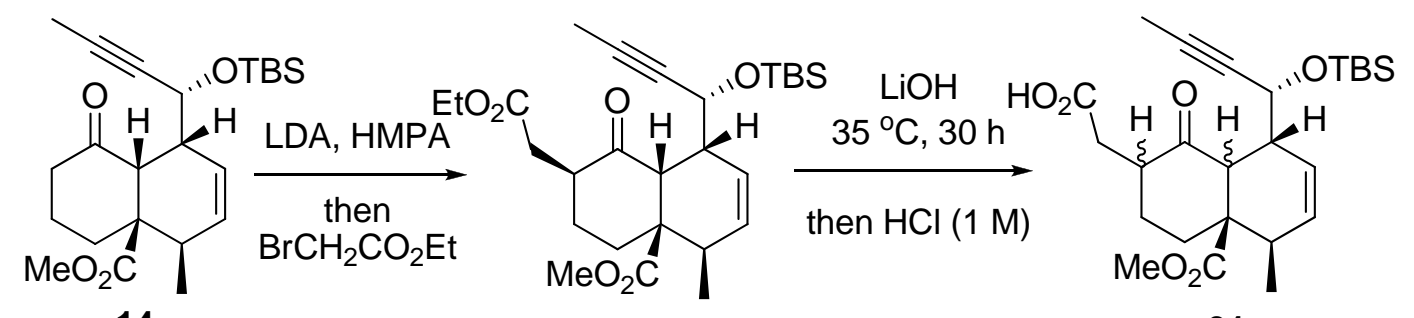
14 30 31

1. $\mathrm{ClCO}_{2}{ }^{i} \mathrm{Bu}, \mathrm{Et}_{3} \mathrm{~N}$ then $\mathrm{CH}_{2} \mathrm{~N}_{2}, \mathrm{Et}_{2} \mathrm{O}$

2. $\mathrm{Rh}_{2}(\mathrm{OAc})_{4}$ PhMe, reflux

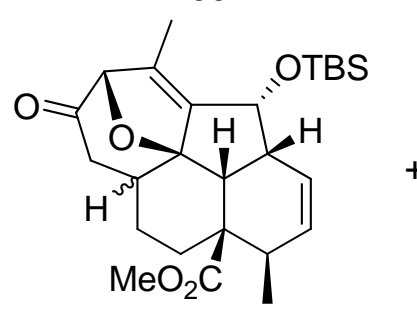

32

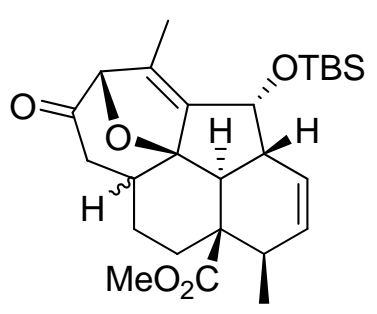

33 


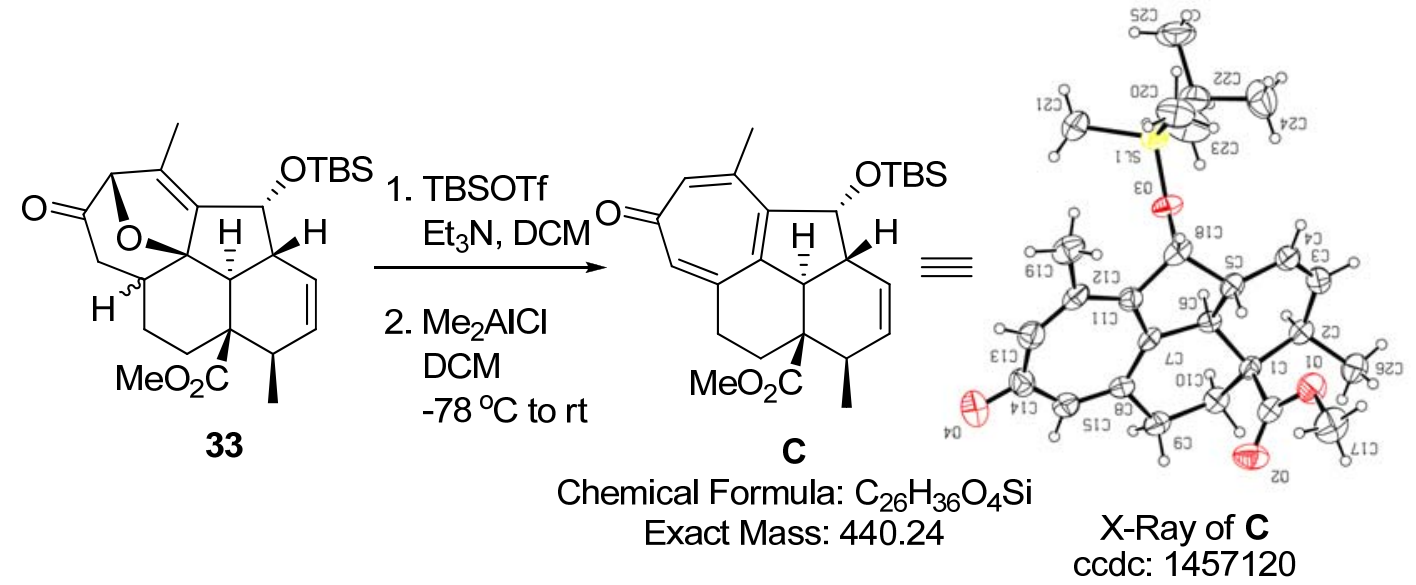

C: ${ }^{1} \mathrm{H}$ NMR $\left(400 \mathrm{MHz}, \mathrm{CDCl}_{3}\right) \delta 6.94(\mathrm{~s}, 2 \mathrm{H}), 5.95(\mathrm{dt}, J=10.0,2.4 \mathrm{~Hz}, 1 \mathrm{H}), 5.51(\mathrm{dt}, J=10.0$ $2.4 \mathrm{~Hz}, 1 \mathrm{H}), 5.20(\mathrm{~d}, J=4.4 \mathrm{~Hz}, 1 \mathrm{H}), 3.62(\mathrm{~s}, 3 \mathrm{H}), 3.38(\mathrm{~d}, J=10.8 \mathrm{~Hz}, 1 \mathrm{H}), 3.23-3.19(\mathrm{~m}, 1 \mathrm{H})$, $3.00-2.81(\mathrm{~m}, 2 \mathrm{H}), 2.65-2.60(\mathrm{~m}, 1 \mathrm{H}), 2.44-2.42(\mathrm{~m}, 1 \mathrm{H}), 2.35(\mathrm{~s}, 3 \mathrm{H}), 1.54(\mathrm{td}, J=13.2,6.0$ $\mathrm{Hz}, 1 \mathrm{H}), 1.10$ (d, J=7.6 Hz, 3H), 0.82 (s, 9H), 0.13 (s, 3H), 0.11 (s, 3H), ${ }^{13} \mathrm{C}$ NMR (100 MHz, $\left.\mathrm{CDCl}_{3}\right) \delta 185.9,173.0,148.8,146.3,144.7,144.0,141.5,141.2,134.9,125.7,77.2,52.9,51.3$, $46.6,45.9,43.2,32.1,30.3,26.0,24.1,18.5,15.4,-3.2,-4.0$.

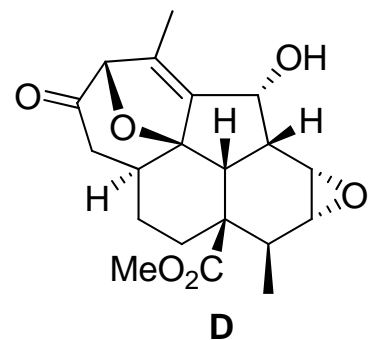

Chemical Formula: $\mathrm{C}_{20} \mathrm{H}_{24} \mathrm{O}_{6}$ Exact Mass: 360.16

D: ${ }^{1} \mathrm{H}$ NMR $\left(400 \mathrm{MHz}, \mathrm{CDCl}_{3}\right) \delta 4.90(\mathrm{~s}, 1 \mathrm{H}), 4.72(\mathrm{~s}, 1 \mathrm{H}), 3.77(\mathrm{~s}, 3 \mathrm{H}), 3.38-3.37(\mathrm{~m}, 1 \mathrm{H})$, $3.29-3.23(\mathrm{~m}, 2 \mathrm{H}), 3.20(\mathrm{t}, J=4.0 \mathrm{~Hz}, 1 \mathrm{H}), 2.75(\mathrm{dd}, J=16.8,7.2 \mathrm{~Hz}, 1 \mathrm{H}), 2.55-2.51(\mathrm{~m}, 1 \mathrm{H})$, $2.50-2.45(\mathrm{~m}, 1 \mathrm{H}), 2.43-2.32(\mathrm{~m}, 3 \mathrm{H}), 1.95(\mathrm{~d}, J=13.6 \mathrm{~Hz}, 1 \mathrm{H}), 1.86(\mathrm{~s}, 3 \mathrm{H}), 1.61-1.44(\mathrm{~m}$, $1 \mathrm{H}), 1.19-1.08(\mathrm{~m}, 1 \mathrm{H}), 0.87(\mathrm{~d}, J=6.8 \mathrm{~Hz}, 3 \mathrm{H})$. 

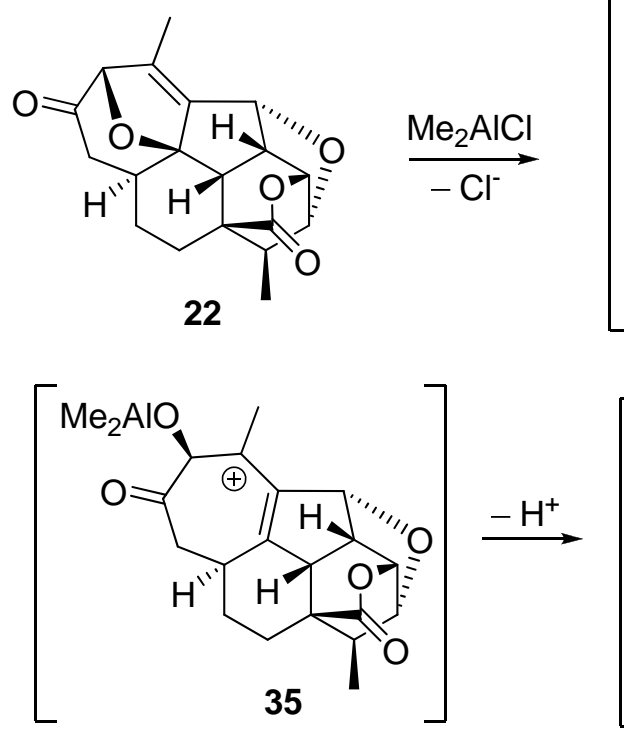

35

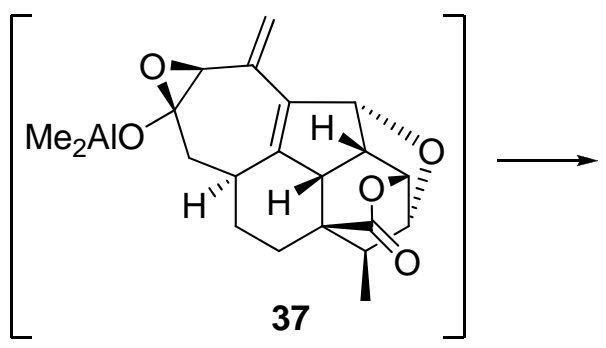

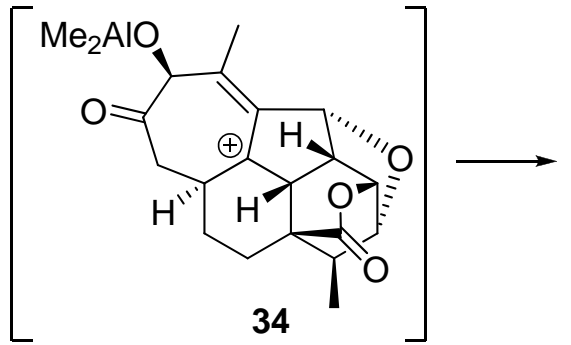

34
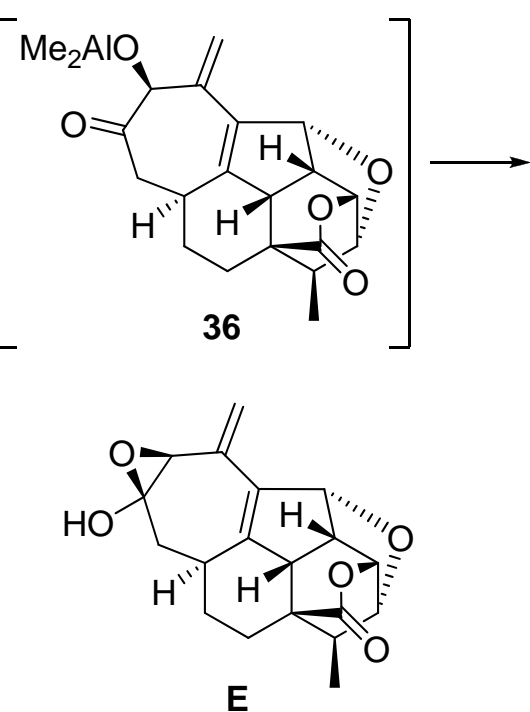

Chemical Formula: $\mathrm{C}_{19} \mathrm{H}_{20} \mathrm{O}_{5}$

Exact Mass: 328.13

E: ${ }^{1} \mathrm{H}$ NMR $\left(400 \mathrm{MHz}, \mathrm{CDCl}_{3}\right) \delta 5.39(\mathrm{~s}, 1 \mathrm{H}), 5.31(\mathrm{~s}, 1 \mathrm{H}), 5.07(\mathrm{t}, J=5.6 \mathrm{~Hz}, 1 \mathrm{H}), 4.88(\mathrm{~d}, J=$ $4.4 \mathrm{~Hz}, 1 \mathrm{H}), 4.08(\mathrm{~s}, 1 \mathrm{H}), 3.93(\mathrm{~d}, J=5.6 \mathrm{~Hz}, 1 \mathrm{H}), 3.24-3.16(\mathrm{~m}, 2 \mathrm{H}), 2.61(\mathrm{dd}, J=14.0,6.0 \mathrm{~Hz}$, 1H), $2.49(\mathrm{~s}, 1 \mathrm{H}), 2.02-1.89(\mathrm{~m}, 3 \mathrm{H}), 1.77-1.65(\mathrm{~m}, 2 \mathrm{H}), 1.61-1.46(\mathrm{~m}, 2 \mathrm{H}), 0.95(\mathrm{~d}, J=7.6$ $\mathrm{Hz}, 3 \mathrm{H}) ;{ }^{1} \mathrm{H}$ NMR $\left(400 \mathrm{MHz}, \mathrm{CDCl}_{3} / \mathrm{D}_{2} \mathrm{O}\right) \delta 5.38(\mathrm{~s}, 1 \mathrm{H}), 5.31(\mathrm{~s}, 1 \mathrm{H}), 5.07(\mathrm{t}, J=5.6 \mathrm{~Hz}, 1 \mathrm{H})$, $4.87(\mathrm{~d}, J=4.4 \mathrm{~Hz}, 1 \mathrm{H}), 4.07(\mathrm{~s}, 1 \mathrm{H}), 3.93(\mathrm{~d}, J=5.6 \mathrm{~Hz}, 1 \mathrm{H}), 3.23-3.16(\mathrm{~m}, 2 \mathrm{H}), 2.61(\mathrm{dd}, J=$ 14.8, 6.0 Hz, 1H), $2.02-1.89(\mathrm{~m}, 3 \mathrm{H}), 1.78-1.67(\mathrm{~m}, 2 \mathrm{H}), 1.60(\mathrm{~s}, 1 \mathrm{H}), 1.50(\mathrm{~d}, J=10.8 \mathrm{~Hz}$, $1 \mathrm{H}), 0.94(\mathrm{~d}, J=7.6 \mathrm{~Hz}, 3 \mathrm{H}) ;{ }^{13} \mathrm{C}$ NMR $\left(150 \mathrm{MHz}, \mathrm{CDCl}_{3}\right) \delta 174.7,145.9,141.7,137.8,118.3$, 88.6, 83.5, 79.6, 79.2, 74.8, 49.0, 45.0, 42.9, 40.6, 38.7, 34.5, 28.1, 23.7, 15.0; HRMS (ESI): $\mathrm{m} / \mathrm{z}$ calcd for $\mathrm{C}_{19} \mathrm{H}_{19} \mathrm{O}_{4}[\mathrm{M}-\mathrm{OH}]^{+}, 311.1278$, found: 311.1282 . 


\section{Comparison of Spectra of Natural and Synthetic Harringtonolide}

Comparison of ${ }^{1} \mathrm{H}$ NMR Spectra of Harringtonolide $\mathbf{1}$ in $\mathrm{CDCl}_{3}$

(a) ${ }^{1} \mathrm{H}$ NMR spectra provided by B. Nay ${ }^{[1]}$

(b) ${ }^{1}$ H NMR spectra provided by W. P. Tang ${ }^{[2]}$

(c) ${ }^{1}$ H NMR spectra of our synthetic 1

(a)
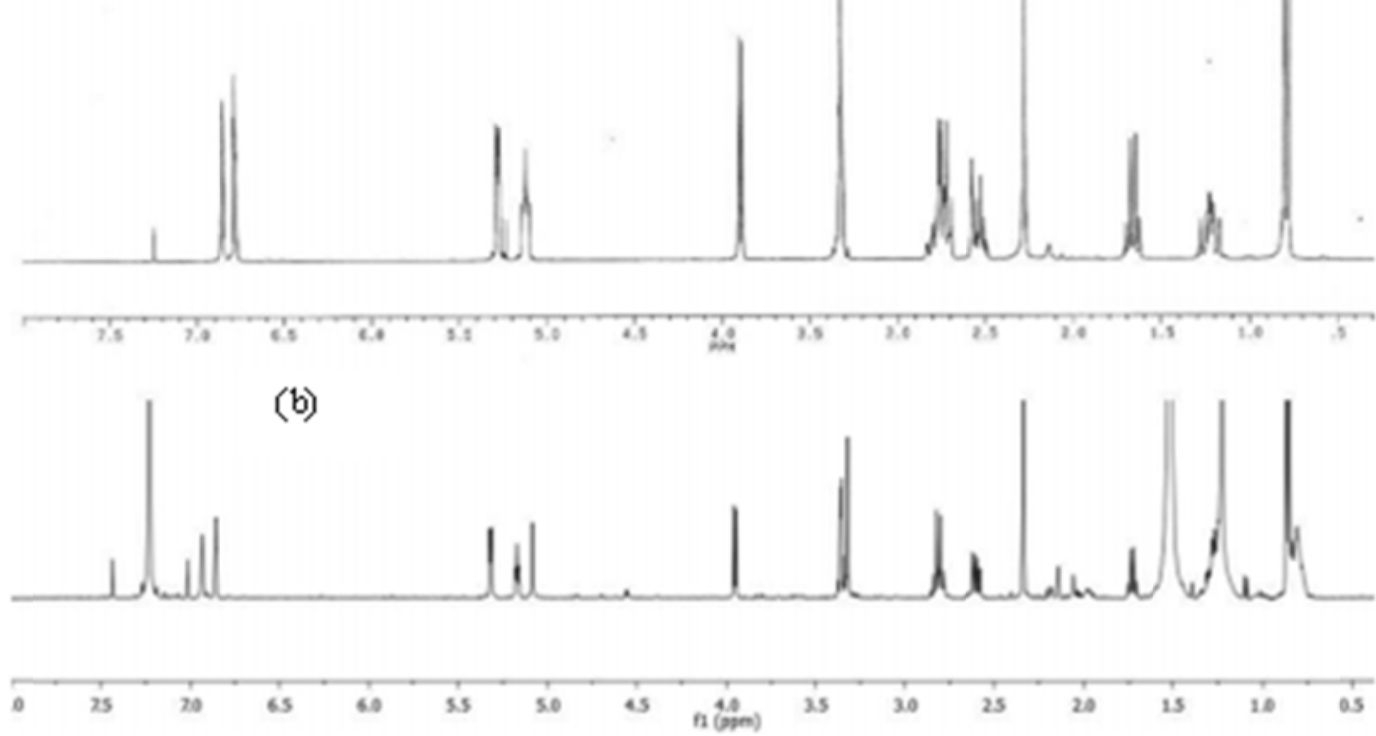

(c)

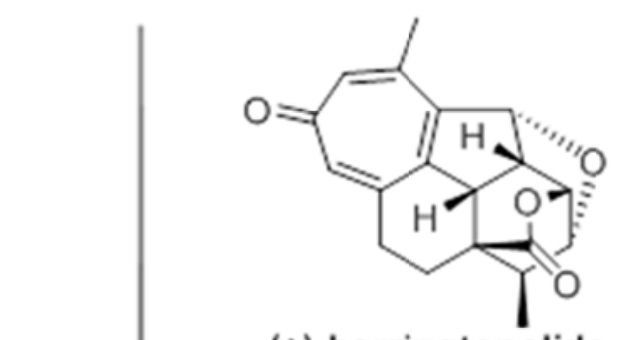

(+)-harringtonolide

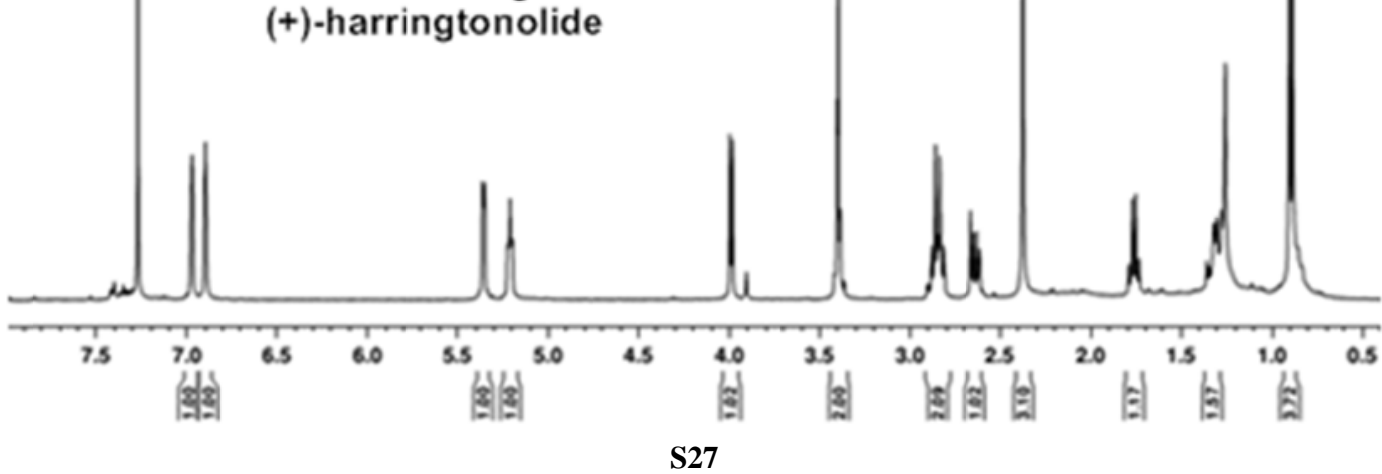


Table S1 ${ }^{1}$ H NMR Spectroscopic Data of Our Synthetic 1, Buta's Natural $\mathbf{1},{ }^{[3]}$ Sun' Natural $1,{ }^{[4]}$ and Tang's Synthetic $\mathbf{1}^{[2]}$ in $\mathrm{CDCl}_{3}$

\begin{tabular}{|c|c|c|c|}
\hline $\begin{array}{c}\delta \mathrm{H} \text { of our synthetic } 1 \\
(400 \mathrm{MHz})\end{array}$ & $\begin{array}{c}\delta \mathrm{H} \text { of lit. natural } \mathbf{1}^{[3]} \\
\qquad(100 \mathrm{MHz})\end{array}$ & $\begin{array}{c}\delta \mathrm{H} \text { of lit. natural } \mathbf{1}^{[4]} \\
\qquad(100 \mathrm{MHz})\end{array}$ & $\begin{array}{c}\delta \mathrm{H} \text { of Tang's synthetic } \mathbf{1}^{[2]} \\
(500 \mathrm{MHz})\end{array}$ \\
\hline $6.97(\mathrm{~s}, 1 \mathrm{H})$ & $6.98(\mathrm{~s}, 1 \mathrm{H})$ & $6.95(\mathrm{~d}, J=2 \mathrm{~Hz}, 1 \mathrm{H})$ & $6.98(\mathrm{~s}, 1 \mathrm{H})$ \\
\hline $6.90(\mathrm{~s}, 1 \mathrm{H})$ & $6.92(\mathrm{~s}, 1 \mathrm{H})$ & $6.77(\mathrm{~d}, J=2 \mathrm{~Hz}, 1 \mathrm{H})$ & $6.91(\mathrm{~s}, 1 \mathrm{H})$ \\
\hline $5.36(\mathrm{t}, J=2.8 \mathrm{~Hz}, 1 \mathrm{H})$ & $5.47(\mathrm{~m}, 1 \mathrm{H})$ & $5.35(\mathrm{q}, 1 \mathrm{H})$ & $5.36(\mathrm{~m}, 1 \mathrm{H})$ \\
\hline $5.21(\mathrm{t}, J=5.2 \mathrm{~Hz}, 1 \mathrm{H})$ & $5.32(\mathrm{~m}, 1 \mathrm{H})$ & $5.19(\mathrm{~m}, 1 \mathrm{H})$ & $5.22(\mathrm{dd}, J=5.3,5.3 \mathrm{~Hz}, 1 \mathrm{H})$ \\
\hline $3.99(\mathrm{~d}, J=5.6 \mathrm{~Hz}, 1 \mathrm{H})$ & $4.00(\mathrm{~m}, 1 \mathrm{H})$ & $3.98(\mathrm{~d}, J=6 \mathrm{~Hz}, 1 \mathrm{H})$ & $4.00(\mathrm{~d}, J=5.6 \mathrm{~Hz}, 1 \mathrm{H})$ \\
\hline $3.42-3.36(\mathrm{~m}, 2 \mathrm{H})$ & $3.51(\mathrm{~m}, 2 \mathrm{H})$ & $3.40(\mathrm{~m}, 2 \mathrm{H})$ & $3.42(\mathrm{~m}, 2 \mathrm{H})$ \\
\hline $2.91-2.82(\mathrm{~m}, 2 \mathrm{H})$ & \multirow{3}{*}{$2.70(\mathrm{~m}, 3 \mathrm{H})$} & $2.85(\mathrm{~m}, 1 \mathrm{H})$ & $2.87(\mathrm{~m}, 1 \mathrm{H})$ \\
\hline \multirow{2}{*}{$2.66-2.60(\mathrm{~m}, 1 \mathrm{H})$} & & $2.75(\mathrm{~m}, 1 \mathrm{H})$ & $2.83(\mathrm{~m}, 1 \mathrm{H})$ \\
\hline & & $2.65(\mathrm{~m}, 1 \mathrm{H})$ & $2.64(\mathrm{dd}, J=13.7,6.5 \mathrm{~Hz}, 1 \mathrm{H})$ \\
\hline $2.37(\mathrm{~d}, J=0.8 \mathrm{~Hz}, 3 \mathrm{H})$ & $2.36(\mathrm{~s}, 3 \mathrm{H})$ & $2.37(\mathrm{~s}, 3 \mathrm{H})$ & $2.38(\mathrm{~s}, 3 \mathrm{H})$ \\
\hline $1.76(\mathrm{q}, J=7.6 \mathrm{~Hz}, 1 \mathrm{H})$ & $1.75(\mathrm{q}, 1 \mathrm{H})$ & $1.74(\mathrm{q}, J=8 \mathrm{~Hz}, 1 \mathrm{H})$ & $1.77(\mathrm{q}, J=7.5 \mathrm{~Hz}, 1 \mathrm{H})$ \\
\hline $1.36-1.28(\mathrm{~m}, 1 \mathrm{H})$ & $1.25(\mathrm{~m}, 1 \mathrm{H})$ & $1.32(\mathrm{~m}, 1 \mathrm{H})$ & $1.31(\mathrm{~m}, 1 \mathrm{H})$ \\
\hline $0.90(\mathrm{~d}, J=7.6 \mathrm{~Hz}, 3 \mathrm{H})$ & $0.90(\mathrm{~d}, 3 \mathrm{H})$ & $0.89(\mathrm{~d}, J=8 \mathrm{~Hz}, 3 \mathrm{H})$ & $0.91(\mathrm{~d}, J=7.6 \mathrm{~Hz}, 3 \mathrm{H})$ \\
\hline
\end{tabular}


Table S2. ${ }^{13} \mathrm{C}$ NMR Spectroscopic Data of Our Synthetic 1, Nay's Natural 1, ${ }^{[1]}$ Buta's Natural 1,${ }^{[3]}$ Sun's Natural $\mathbf{1},{ }^{[4]}$ and Tang's Synthetic $\mathbf{1}^{[2]}$ in $\mathrm{CDCl}_{3}$

\begin{tabular}{|c|c|c|c|c|}
\hline $\begin{array}{c}\delta \mathrm{C}(\mathrm{I}) \text { of our } \\
\text { synthetic } \mathbf{1}\end{array}$ & $\begin{array}{c}\delta \mathrm{C}(\mathrm{II}) \text { of lit. } \\
\text { natural } \mathbf{1}^{[1]}\end{array}$ & $\begin{array}{c}\delta \mathrm{C} \text { (III) of lit. } \\
\text { natural } \mathbf{1}^{[3]}\end{array}$ & $\begin{array}{c}\delta \mathrm{C}(\mathrm{IV}) \text { of lit. } \\
\text { natural } \mathbf{1}^{[4]}\end{array}$ & $\begin{array}{l}\delta C \text { of Tang's } \\
\text { synthetic } 1^{[2]}\end{array}$ \\
\hline 186.5 & 186.2 & 186.4 & 186.9 & 186.3 \\
\hline 173.5 & 173.3 & 173.5 & 173.0 & 173.3 \\
\hline 145.8 & 145.7 & 145.9 & 145.9 & 145.7 \\
\hline 145.6 & 145.5 & 145.7 & 145.2 & - \\
\hline 145.0 & 144.9 & 145.0 & 145.0 & 144.8 \\
\hline 143.6 & 143.4 & 143.6 & 143.4 & - \\
\hline 141.6 & 141.3 & 141.5 & 141.5 & 141.5 \\
\hline 139.3 & 139.0 & 139.1 & 139.4 & 139.1 \\
\hline 86.0 & 85.9 & 85.5 & 86.0 & 85.9 \\
\hline 80.0 & 79.8 & 80.0 & 79.6 & 79.9 \\
\hline 79.7 & 79.5 & 80.0 & 78.2 & 79.6 \\
\hline 49.9 & 49.7 & 49.9 & 49.7 & 49.8 \\
\hline 45.8 & 45.6 & 43.8 & 45.5 & 45.7 \\
\hline 41.8 & 41.6 & 41.7 & 41.5 & 41.7 \\
\hline 40.0 & 39.8 & 40.0 & 39.7 & 39.9 \\
\hline 32.3 & 32.1 & 32.3 & 31.8 & 32.2 \\
\hline 23.9 & 23.7 & 23.8 & 23.3 & 23.7 \\
\hline 22.4 & 22.2 & 22.3 & 22.0 & 22.3 \\
\hline 14.7 & 14.6 & 14.7 & 14.2 & 14.6 \\
\hline
\end{tabular}

\section{References:}

[1] L. Evanno, A. Jossang, J. Nguyen-Pouplin, D. Delaroche, P. Herson, M. Seuleiman, B. Bodo, B. Nay, Planta Med. 2008, 74, 870-872.

[2] M. Zhang, N. Liu, W. P. Tang, J. Am. Chem. Soc. 2013, 135, 12434-12438.

[3] J. G. Buta, J. L. Flippen, W. R. Lusby, J. Org. Chem. 1978, 43, 1002-1003.

[4] N. J. Sun, Z. Xue, X. T. Liang, L. Huang, Acta Pharm. Sin. 1979, 14, 39-44. 
HPLC Data of ( \pm )-8 and (-)-8

\section{Empower}

进样综合报告 报告

\section{Ensoltwar}

用户名称: System

项目名称: a2015

\begin{tabular}{|c|c|c|c|}
\hline \multicolumn{4}{|c|}{ 样品信息 } \\
\hline 样品名称: & zhj-race20151009 & 采集者: & System \\
\hline 样品类型: & 未知 & 采集时间: & 2015-10-8 17:11:05 \\
\hline 瓶号: & 1 & 采集方法组: & zhjrace \\
\hline 进样次数: & & 处理日期: & $2015-10-8$ 17:38:33 \\
\hline 进样体积: & 5.00 ul & 处理方法: & zhjrace20151009 \\
\hline $\begin{array}{l}\text { 运行时间: } \\
\text { 样品维名称: }\end{array}$ & 60.0 Minutes & $\begin{array}{l}\text { 通道名称: } \\
\text { 处理通道注泽: }\end{array}$ & $\begin{array}{l}\text { Wuln Ch1 } \\
\text { PDA } 230.0 \text { 纳米 }\end{array}$ \\
\hline
\end{tabular}

自动标尺色谱图

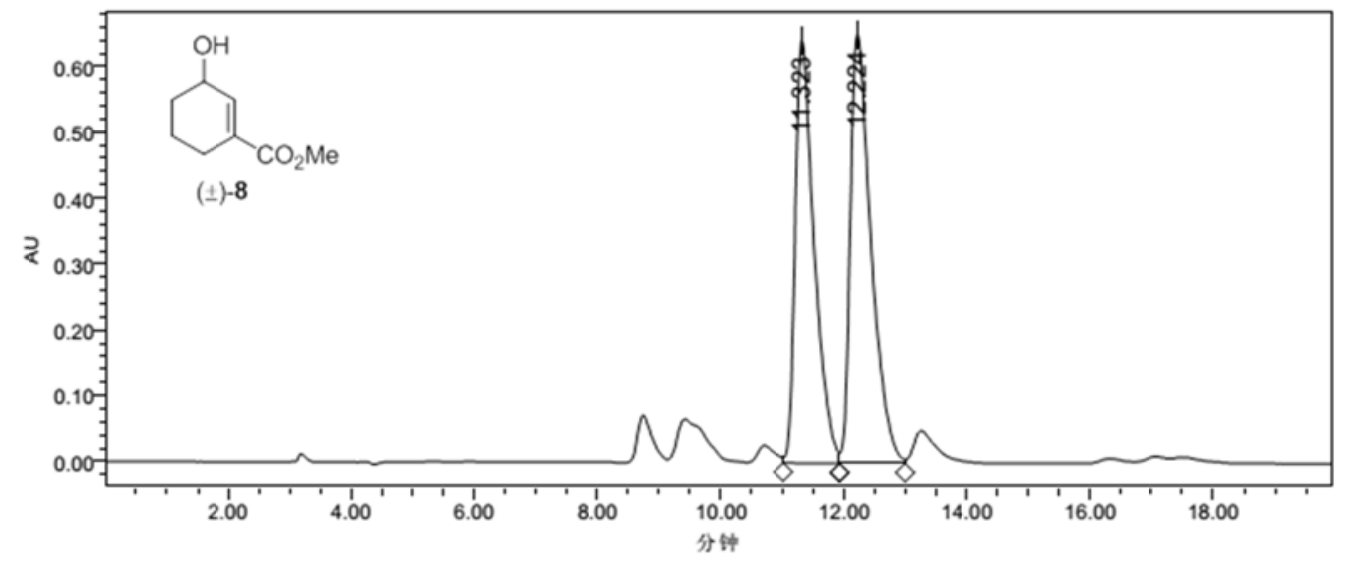

SampleName zhj-race20151009; Vial 1; Injection 4; Channel W2996 ; Date Acquired 2015-10-8 17:11:05 处理通道: PDA 230.0 纳米

\begin{tabular}{|l|c|c|c|c|c|}
\hline & 处理通道 & $\begin{array}{c}\text { 保留时间 } \\
\text { (分钟 }\end{array}$ & 面积 & \% 面积 & 峰高 \\
\hline 1 & PDA 230.0 纳米 & 11.323 & 13789851 & 47.43 & 643009 \\
\hline 2 & PDA 230.0 纳米 & 12.224 & 15283185 & 52.57 & 653100 \\
\hline
\end{tabular}




\begin{tabular}{|c|c|c|c|}
\hline \multicolumn{4}{|c|}{ 样品信息 } \\
\hline 样品名船 & zhj-zif (RuH30g) & 采集者: & System \\
\hline 样品头为; & 末知 & 采集时间: & $2015-10-21 \quad 15: 32.05$ \\
\hline 稳昼: & 1 & 罙集江淮: & zhirace \\
\hline 进佯欢频 & 1 & 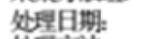 & $2015-10-21 \quad 16: 00: 08$ \\
\hline 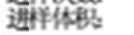 & 5.00 ul & 处祊法 & zhjzjtRuH30g \\
\hline 遥行推: & 60.0 Minutes & 通选名保: & Wvin Ch1 \\
\hline 样噪组名滕: & & 处步通这注䄳: & PDA 230.0 纯米 \\
\hline
\end{tabular}

自动标尺色㳻图

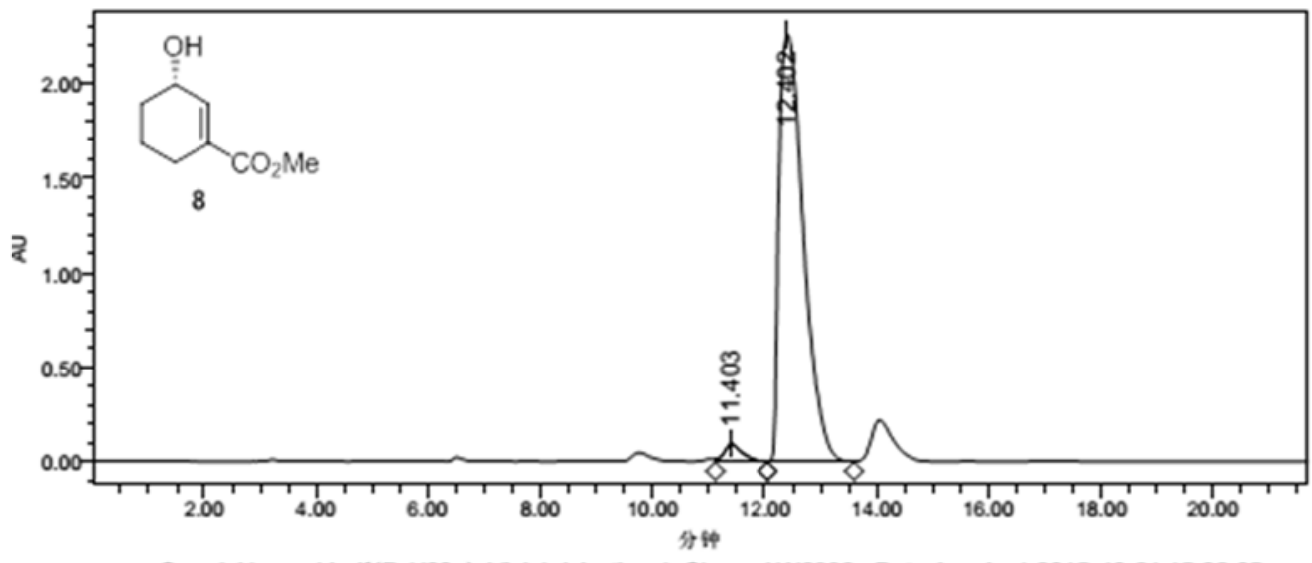

— SampleName żj-zjf (RuH30g); Vial 1; Injection 1; Channel W2996 ; Date Acquired 2015-10-21 15:32:05 处理通道: PDA 230.0 纳米

\begin{tabular}{|c|c|c|c|c|c|}
\hline & 琁五进道 & 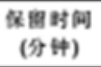 & W 108 & \% 酶 & 냐해 \\
\hline 1 & PDA 230.0 蛸米 & 11.403 & 2117865 & 2.99 & 94087 \\
\hline 2 & PDA 230.0 納米 & 12.402 & 68773533 & 97.01 & 2262600 \\
\hline
\end{tabular}

(-)-8 
NMR Spectra of the Intermediates and (+)-Harringtonolide

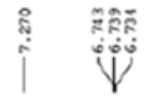

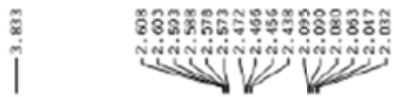

$\mathrm{CHO}_{\mathrm{CO}_{2} \mathrm{Me}}$

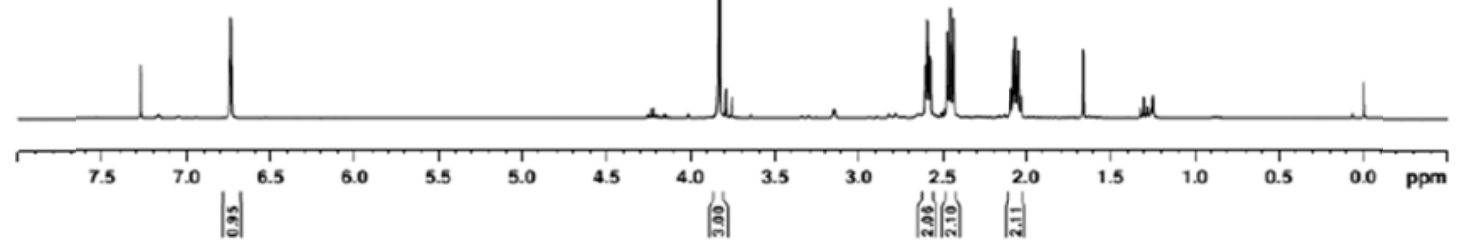

|⿱宀⿻心㇒山<smiles>COC(=O)C1=CC(=O)CCC1</smiles>

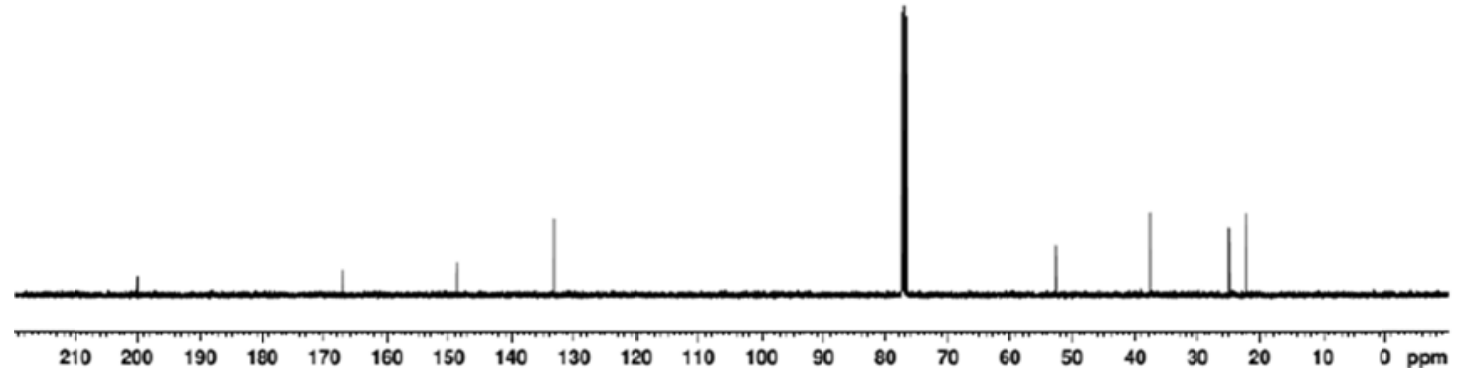




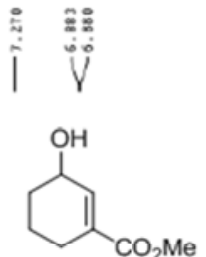

$( \pm)-8$

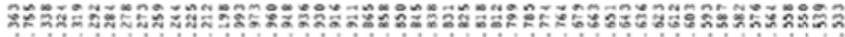

-

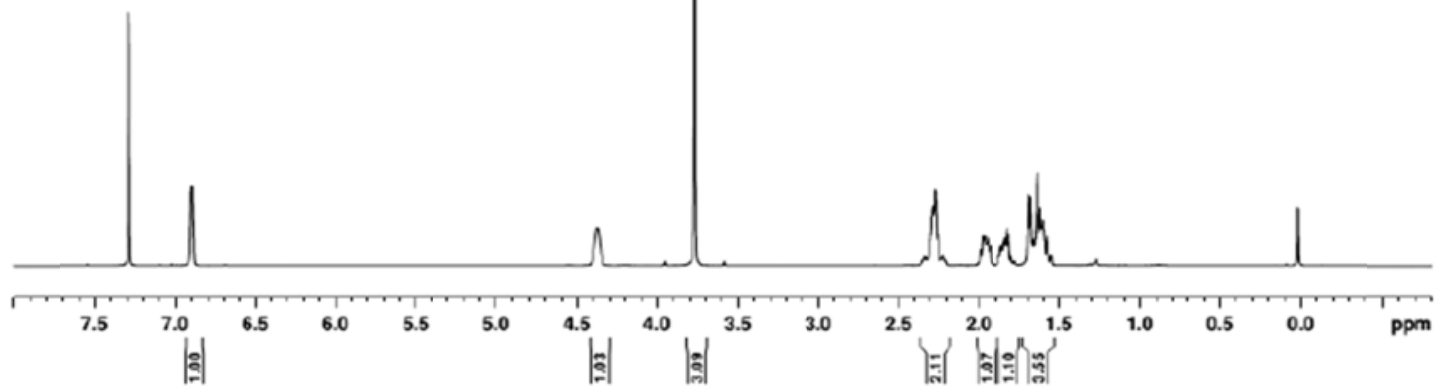

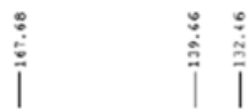

棺<smiles>COC(=O)C1=CC(O)CCC1</smiles>

$( \pm)-8$

$\begin{array}{lllllllllllllllllllllll}210 & 200 & 190 & 180 & 170 & 160 & 150 & 140 & 130 & 120 & 110 & 100 & 90 & 80 & 70 & 60 & 50 & 40 & 30 & 20 & 10 & 0 & \text { ppm }\end{array}$ 


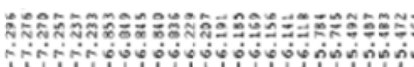

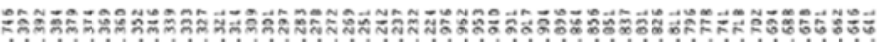

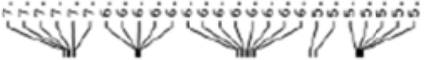
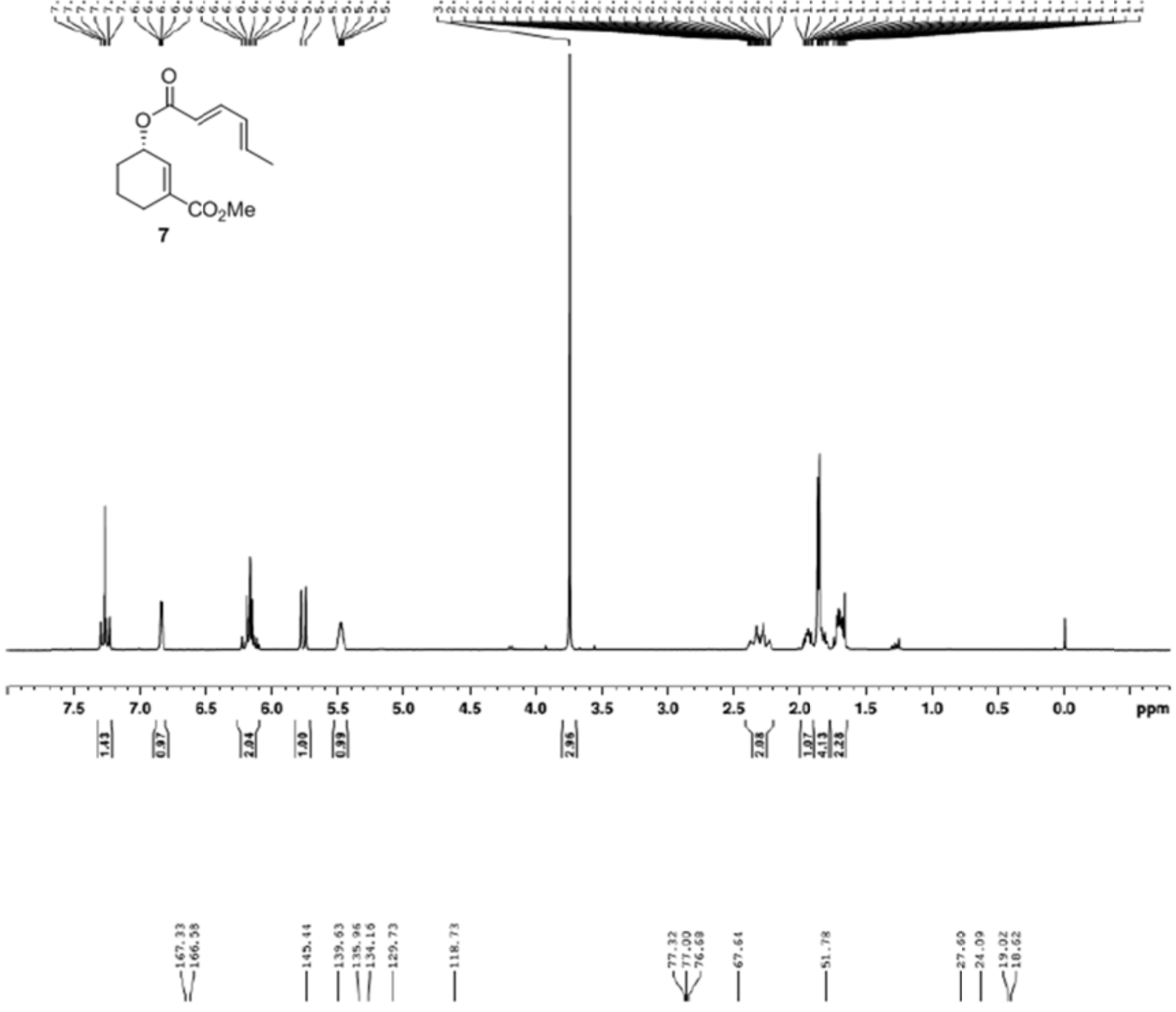<smiles>C/C=C/C=C/C(=O)O[C@H]1C=C(C(=O)OC)CCC1</smiles>

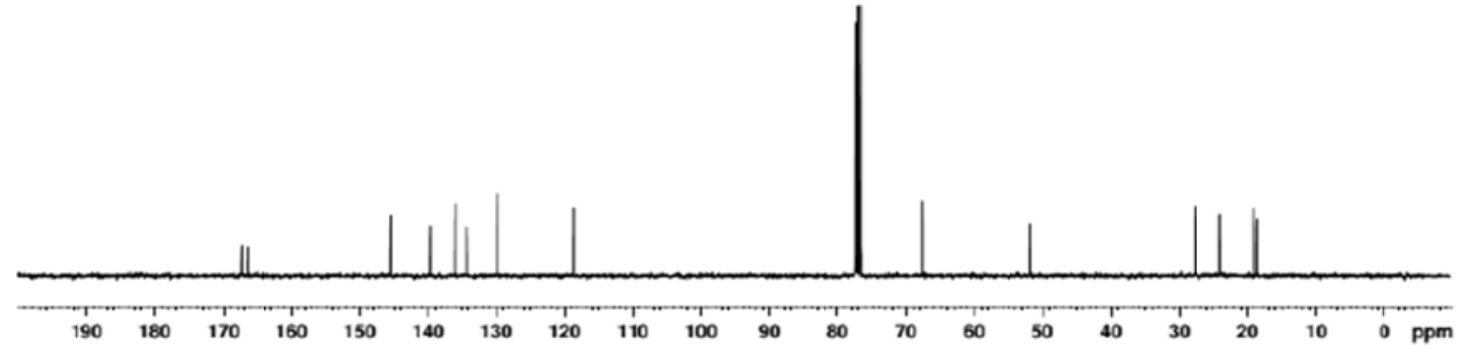



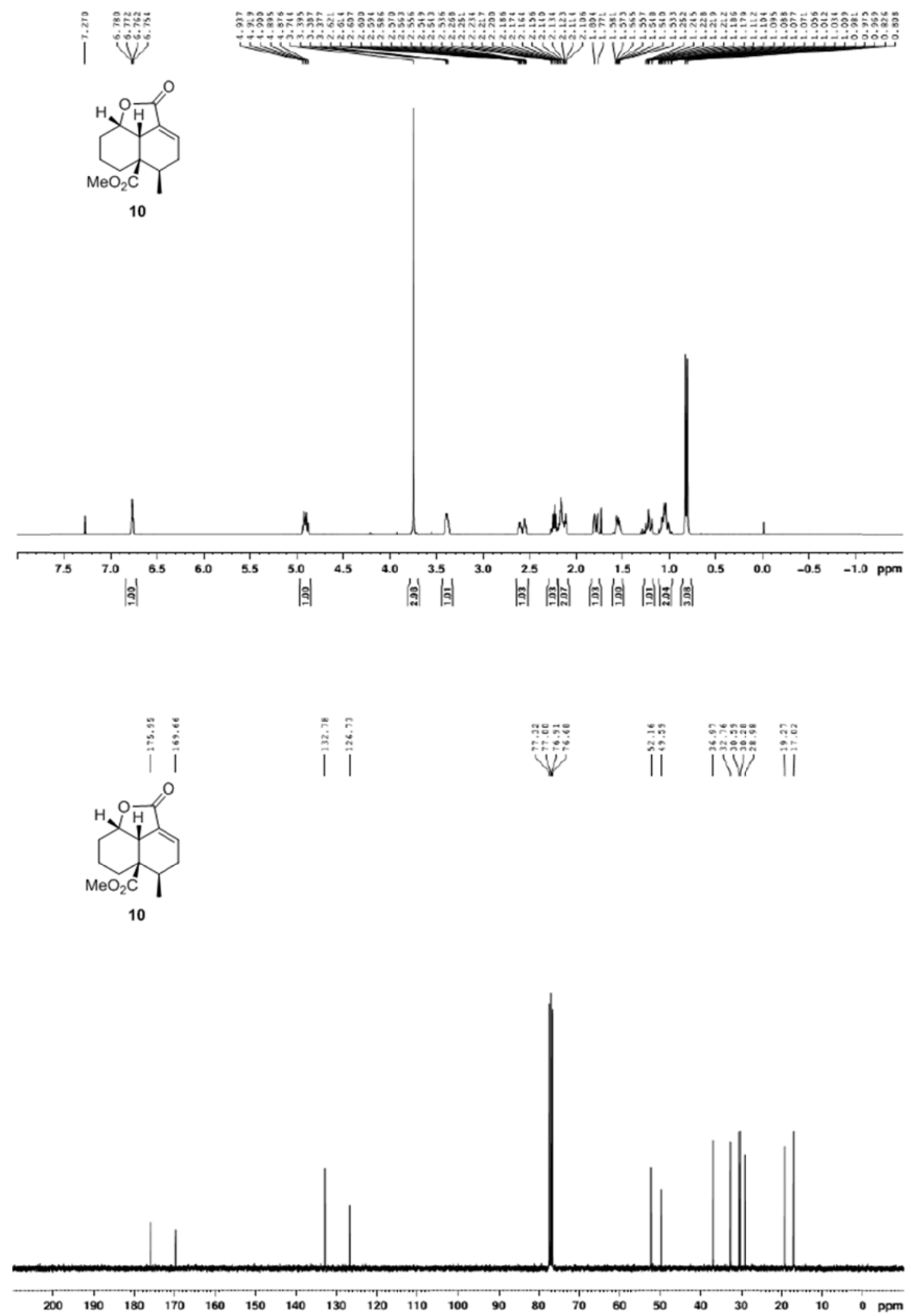
Mixture of 10 and 10’:

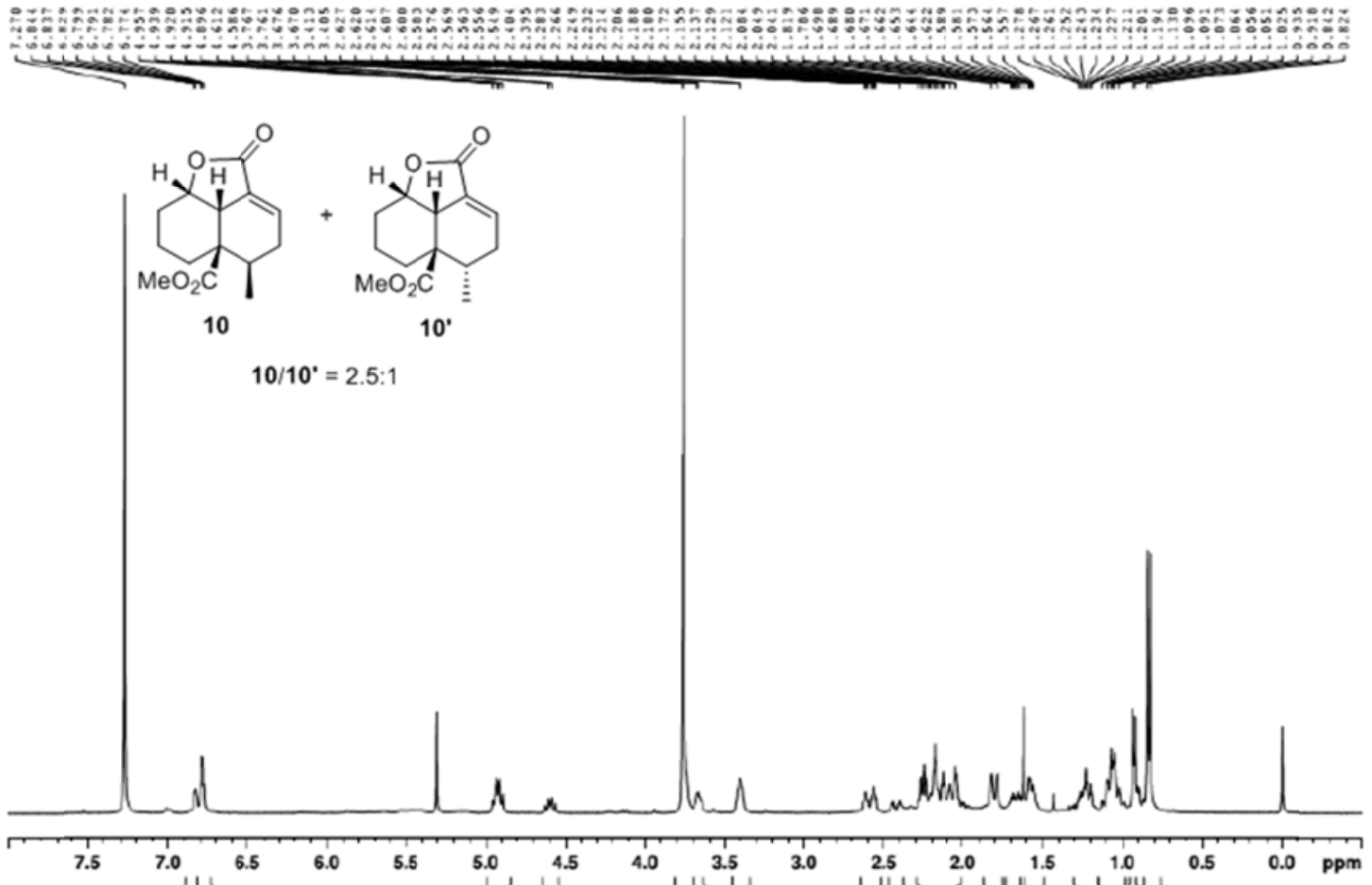

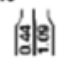
| 8 : 国络

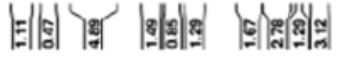



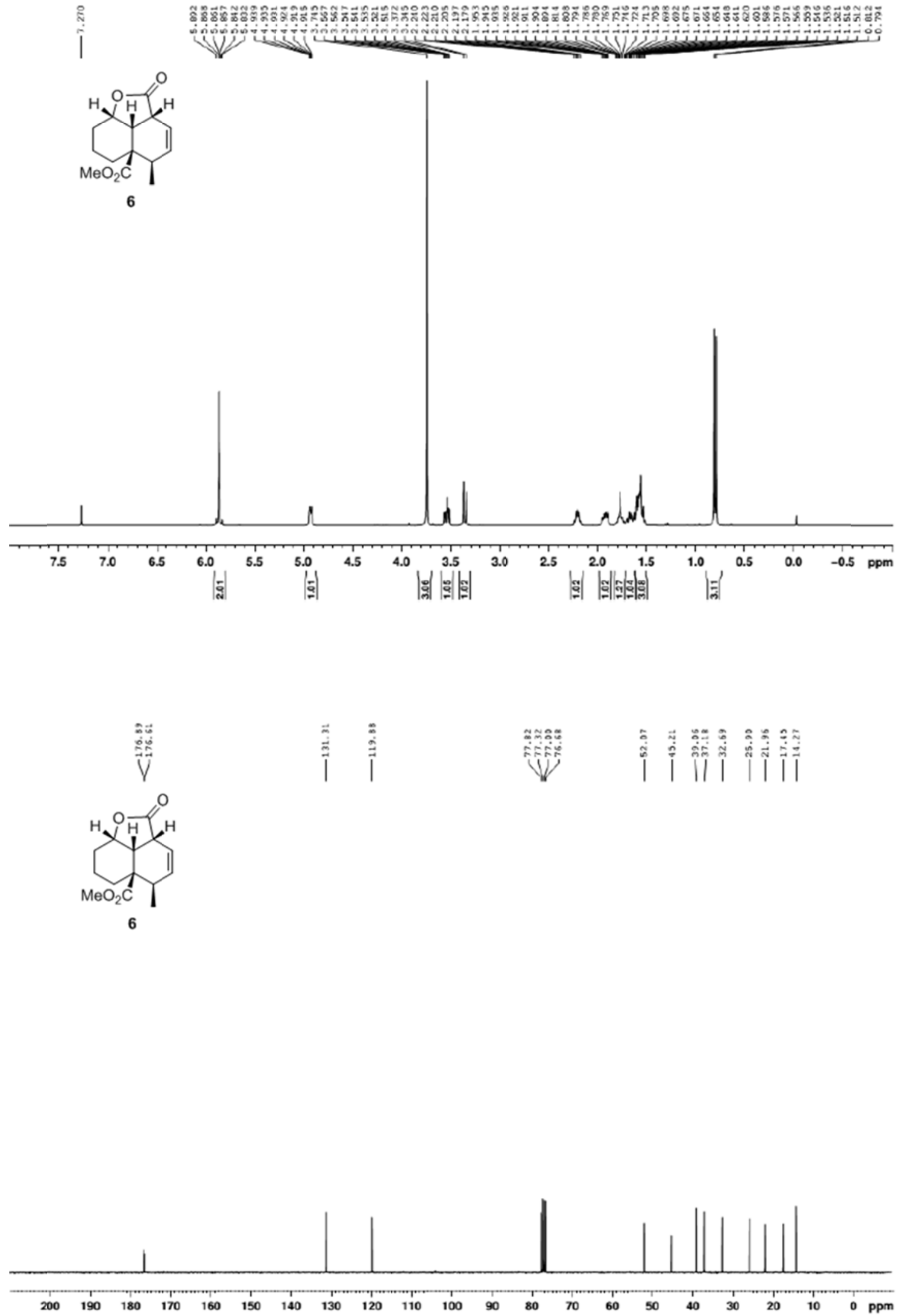


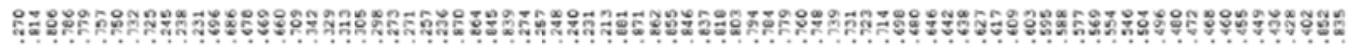

-

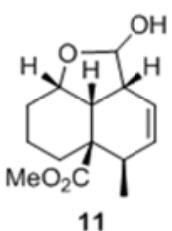

11
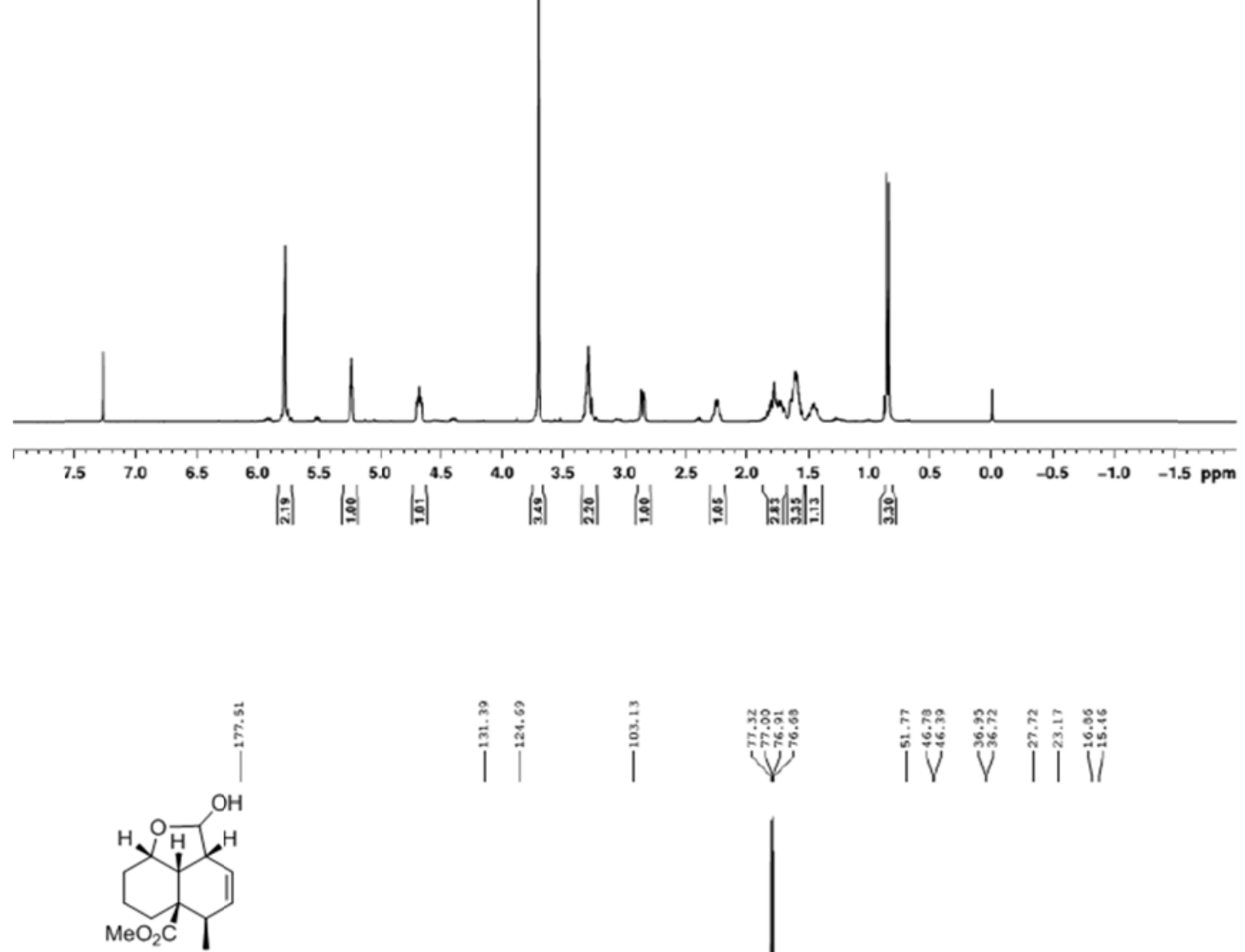

11

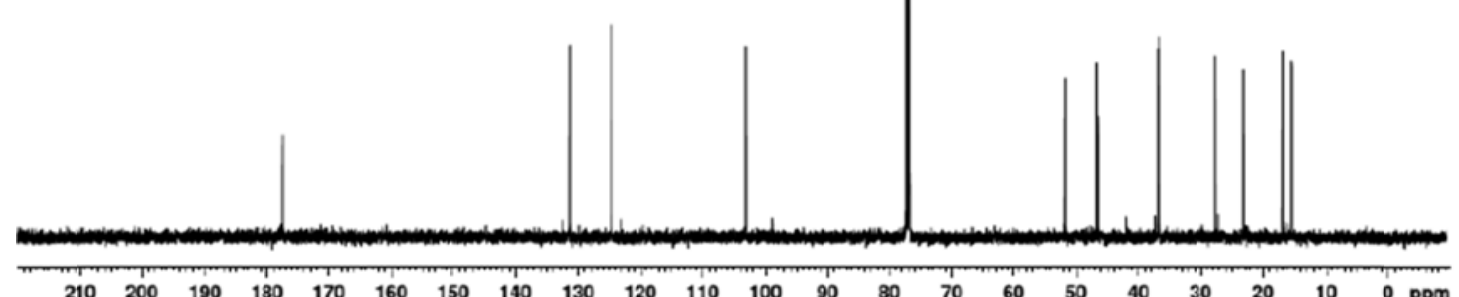



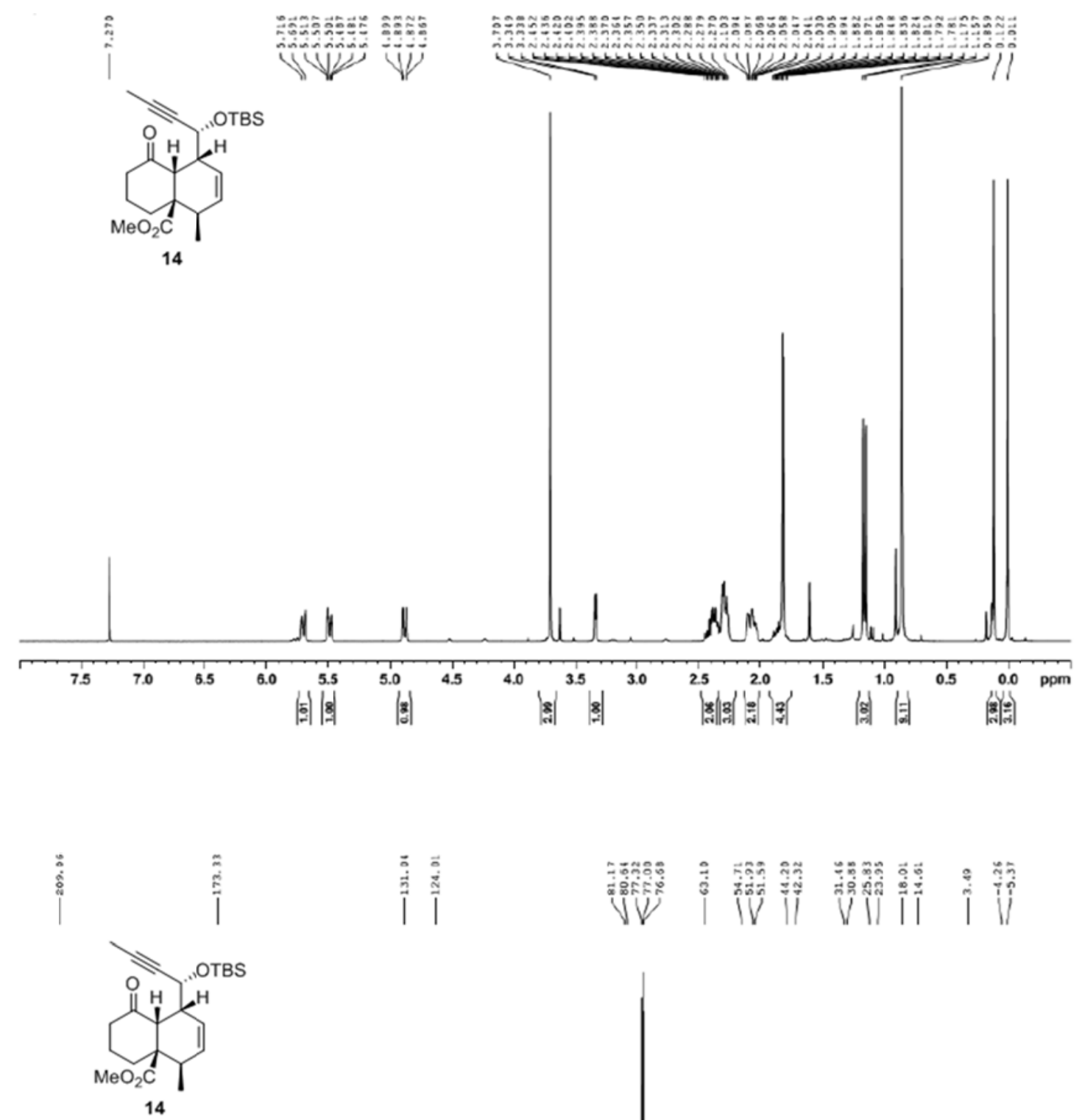

|
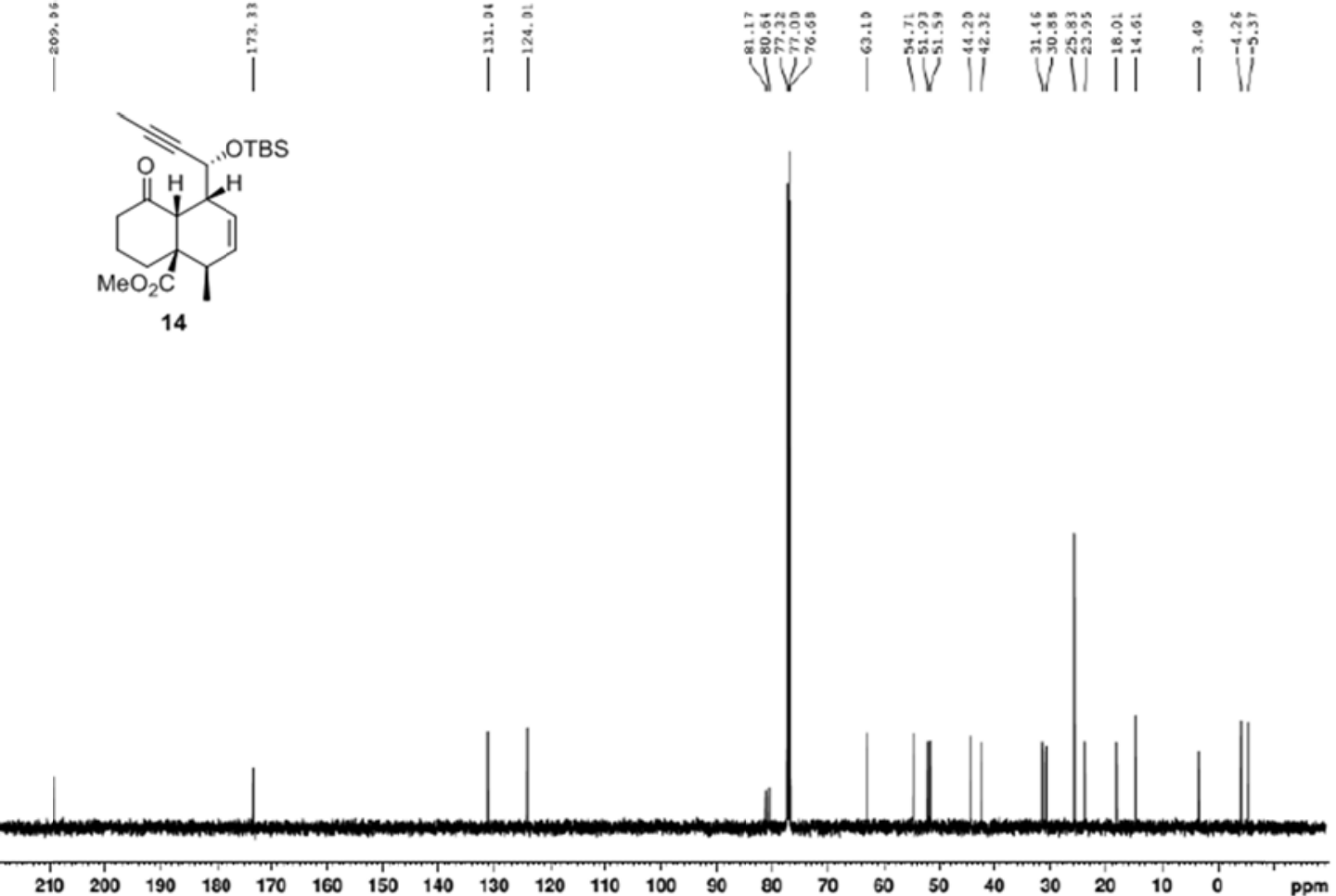

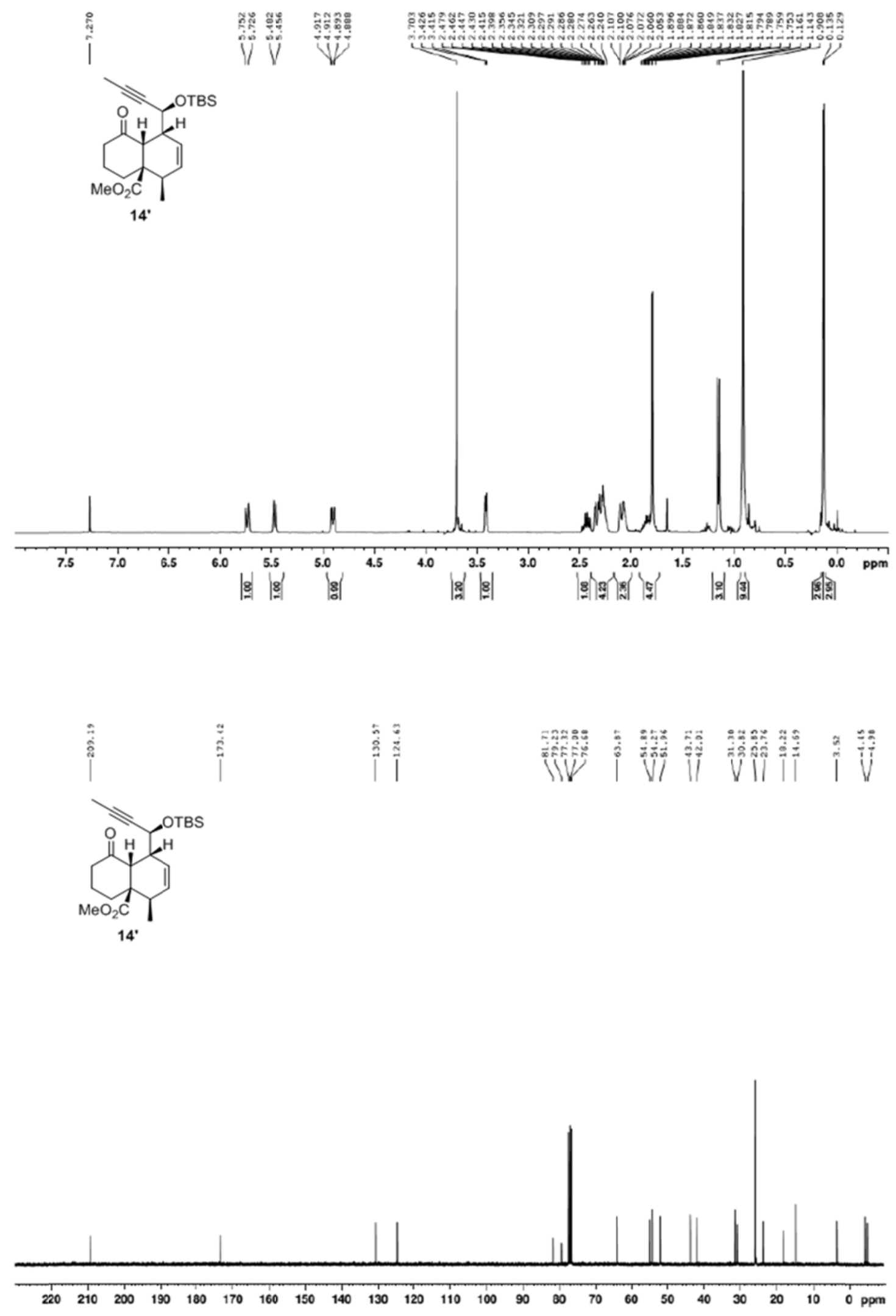

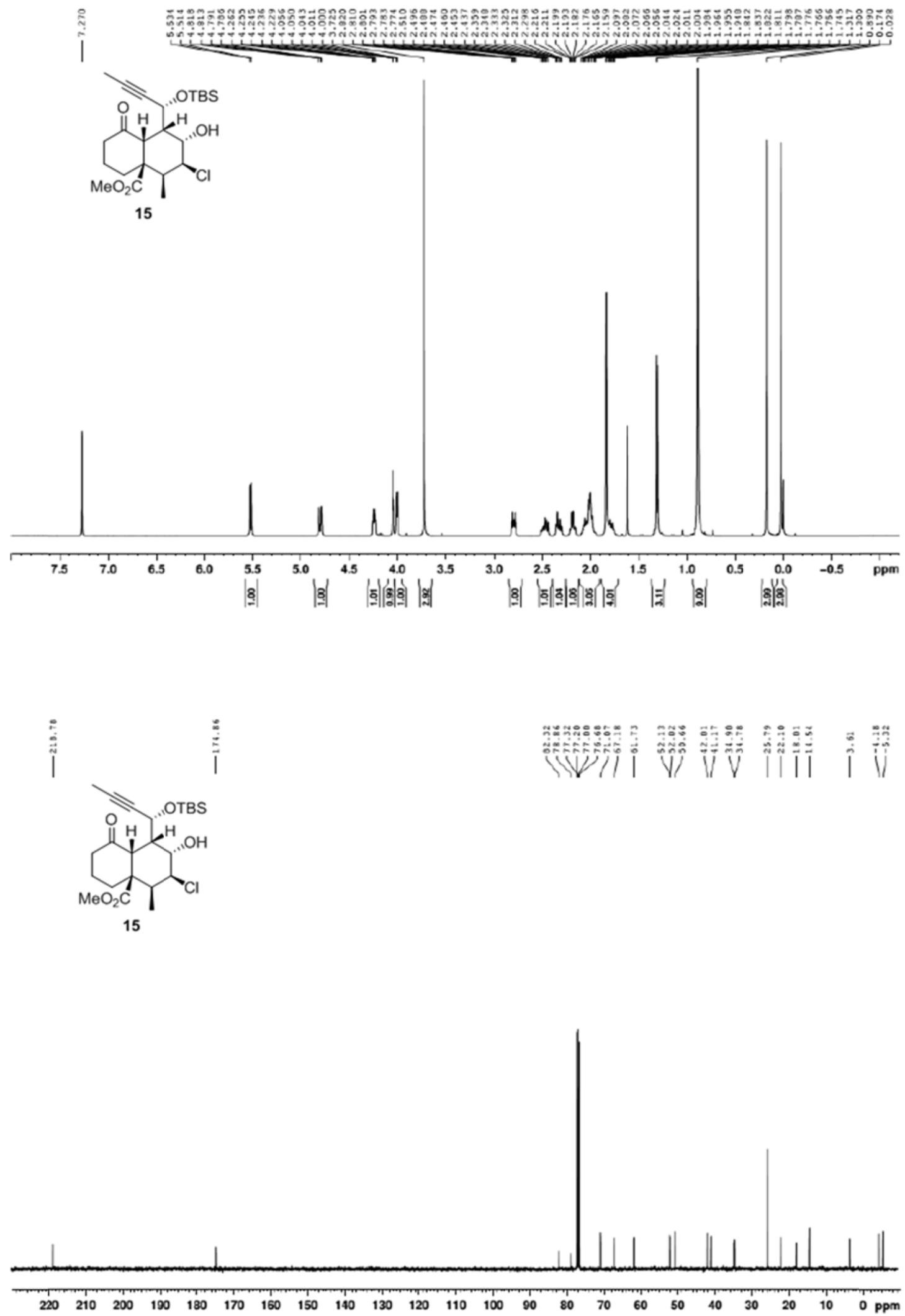

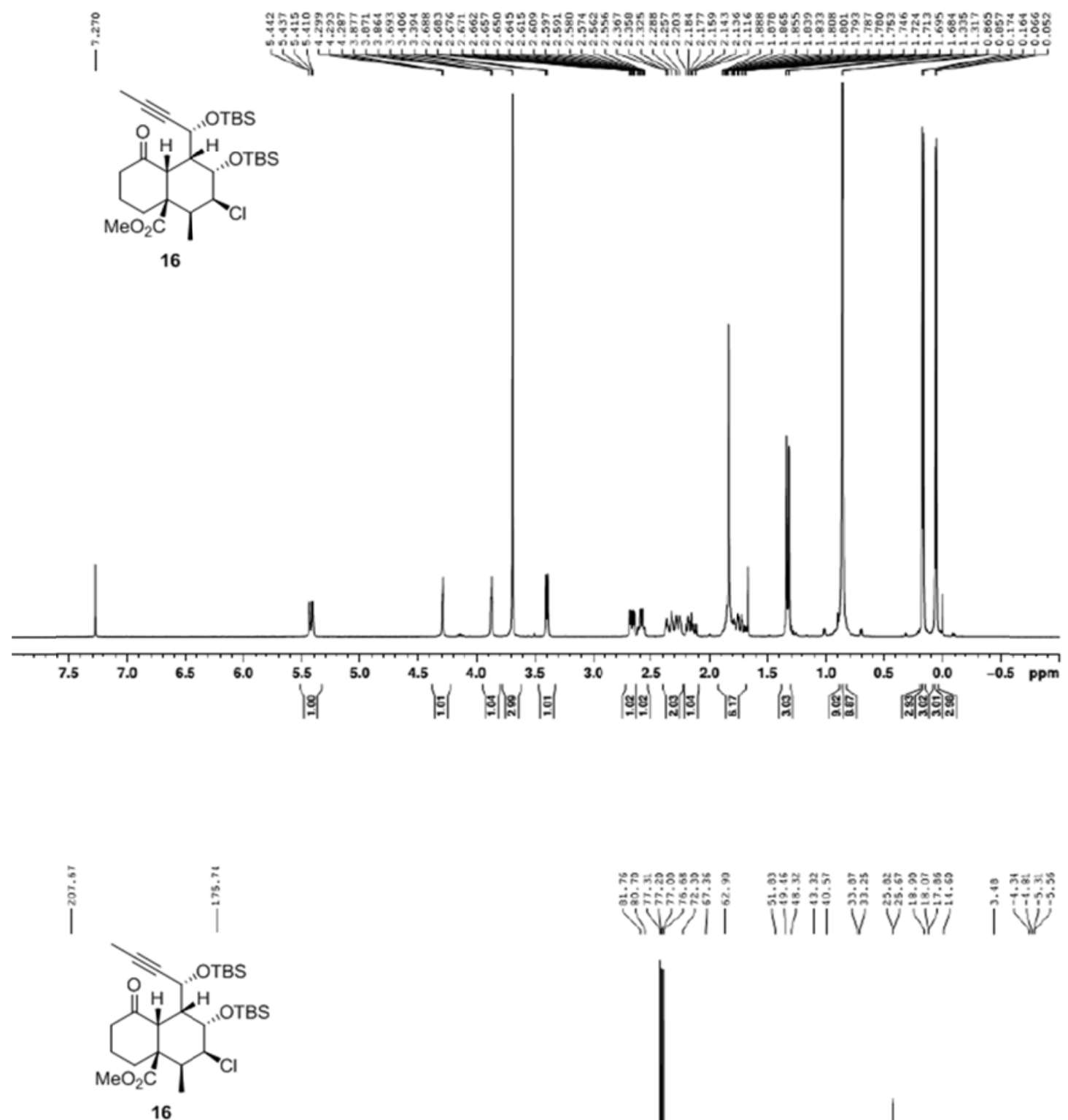

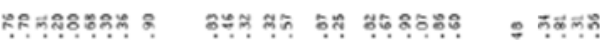

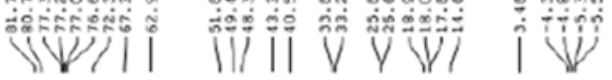

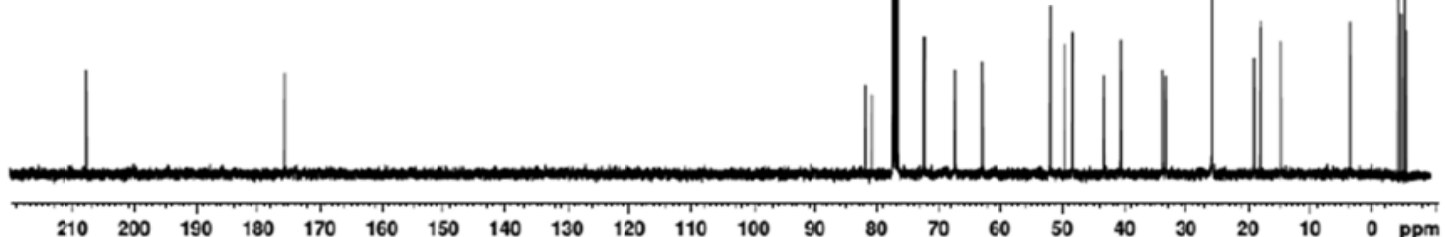



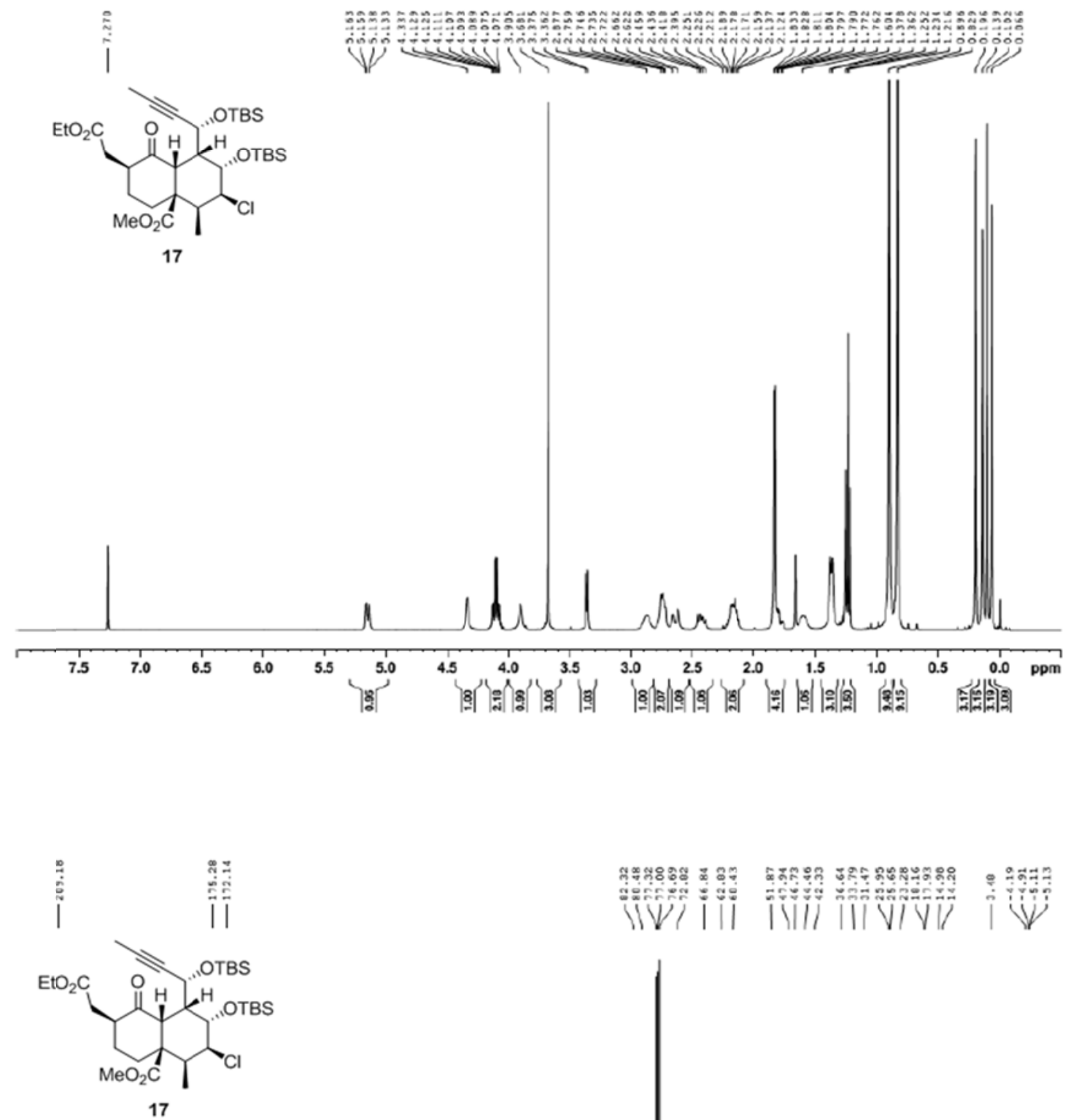

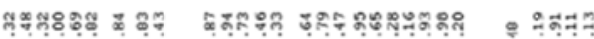
IVVi |i | 

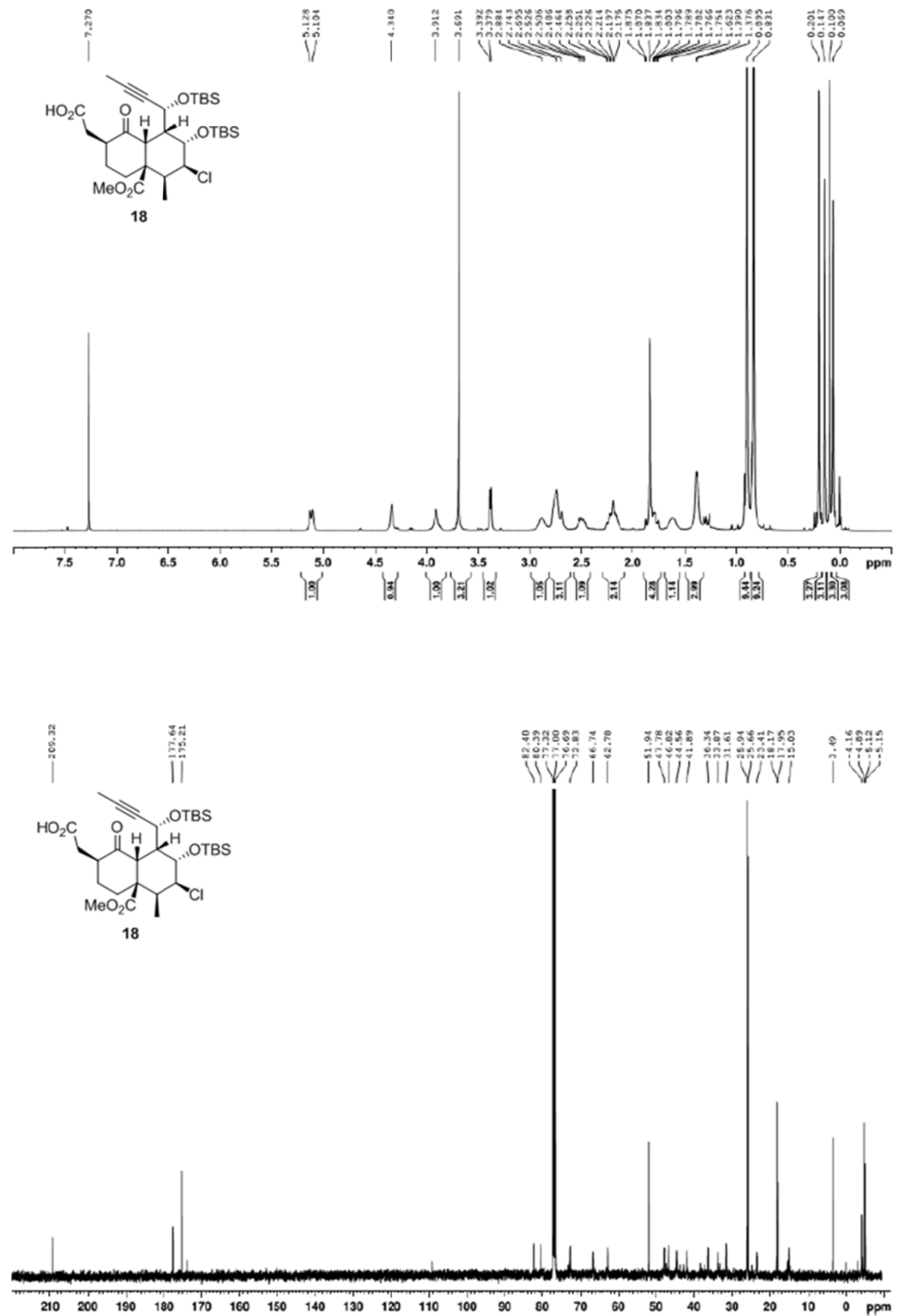

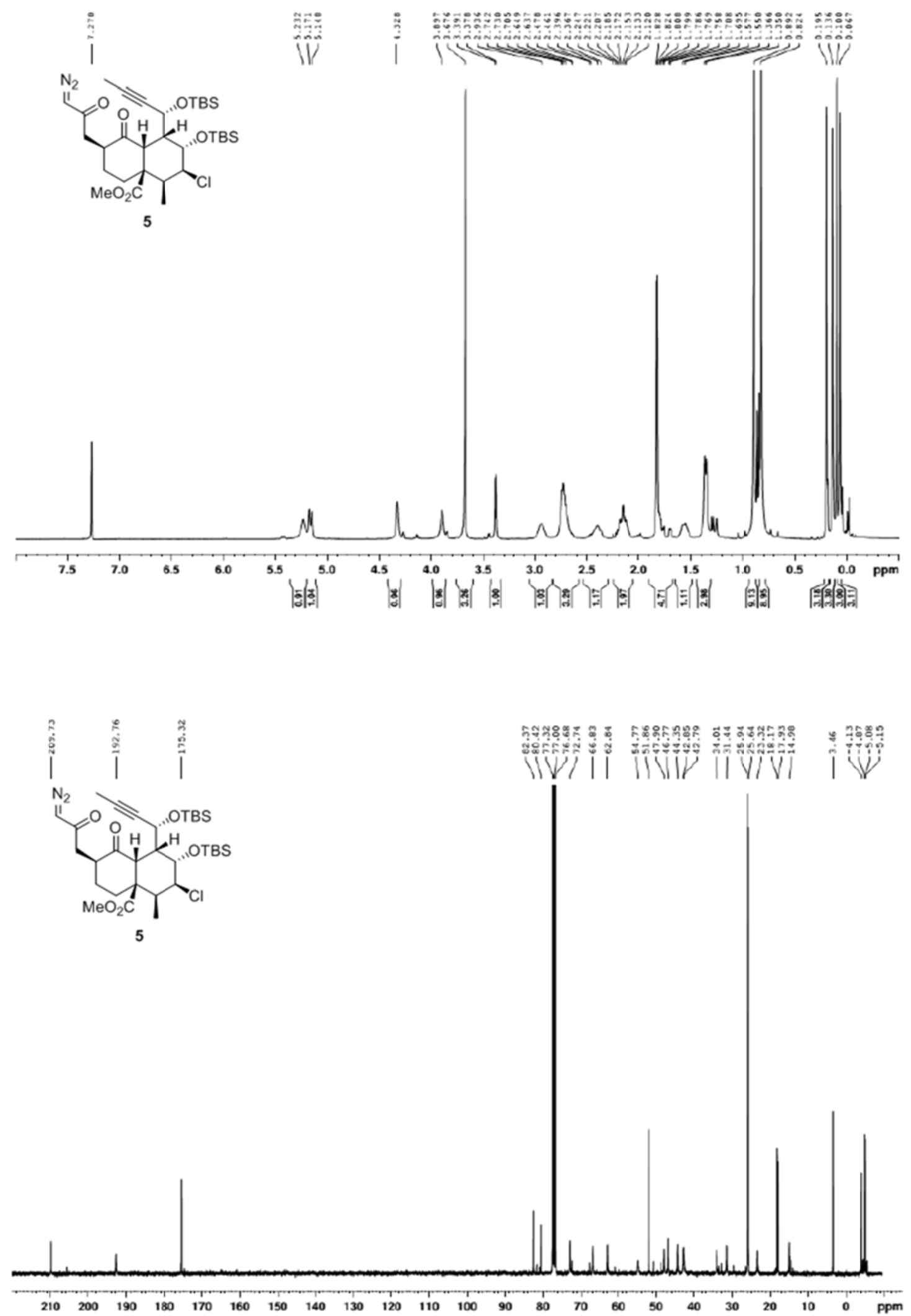

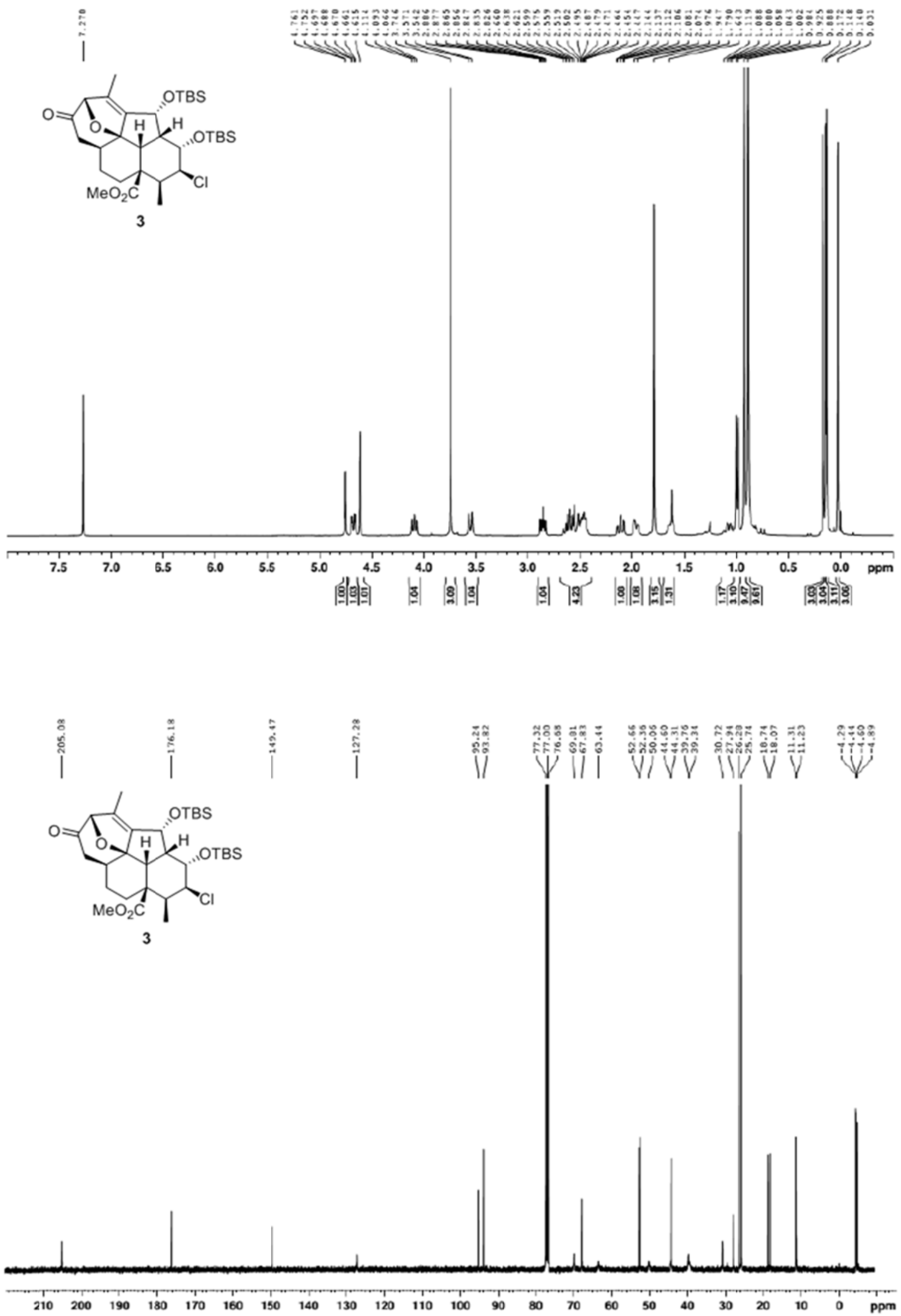


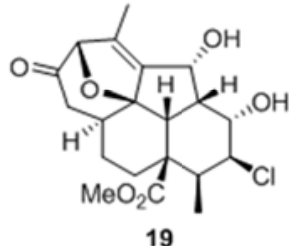

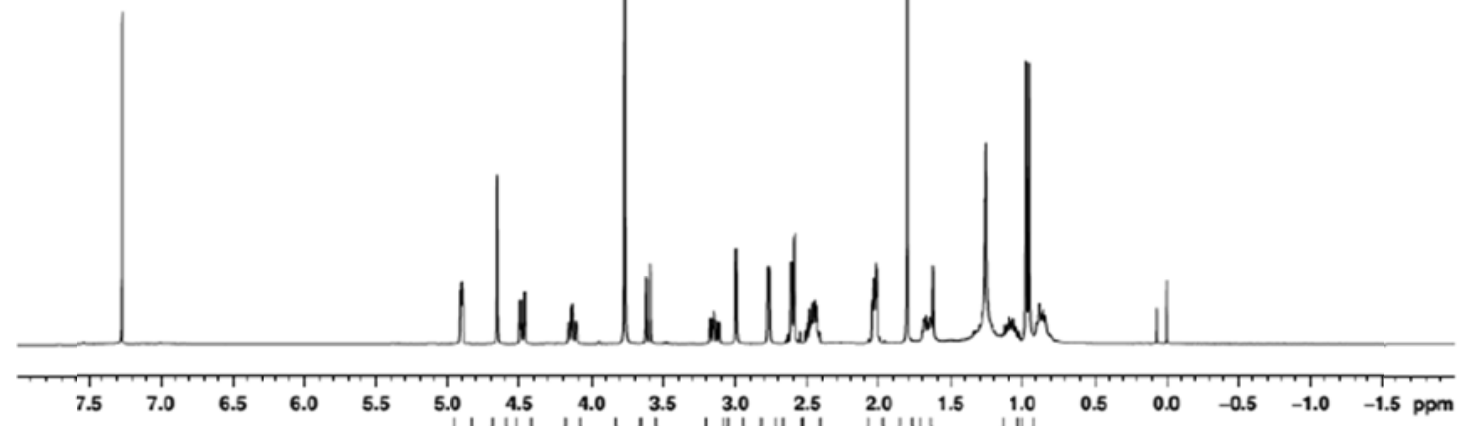

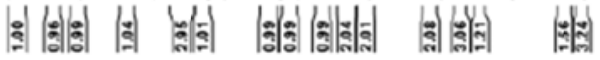

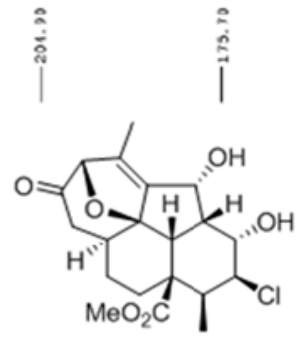

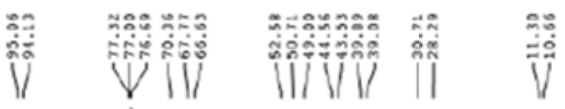

19

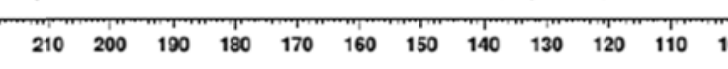



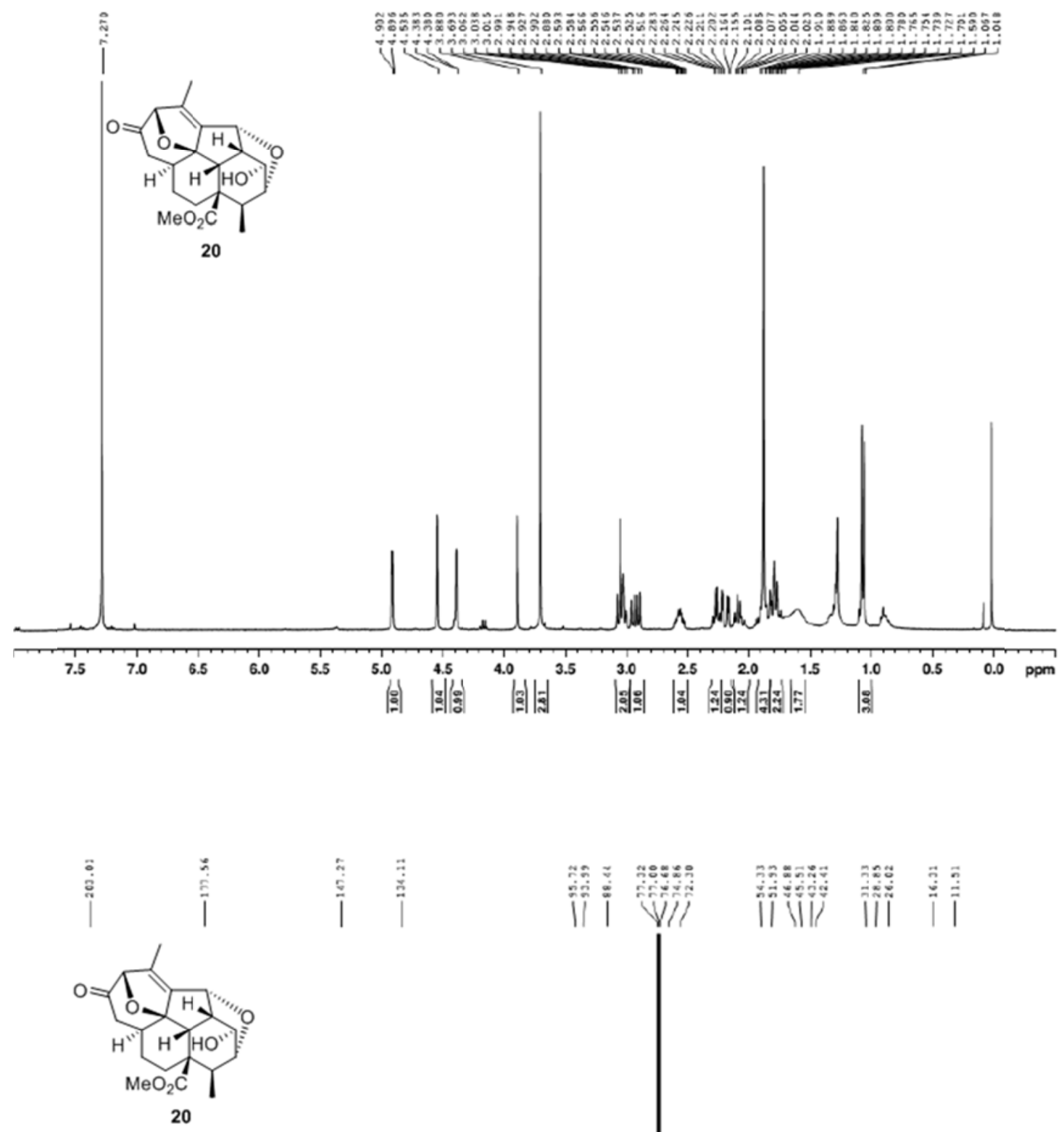

||

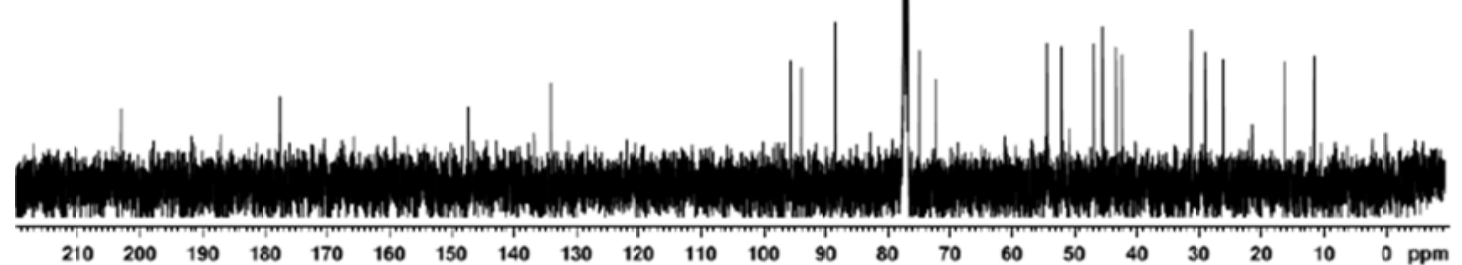



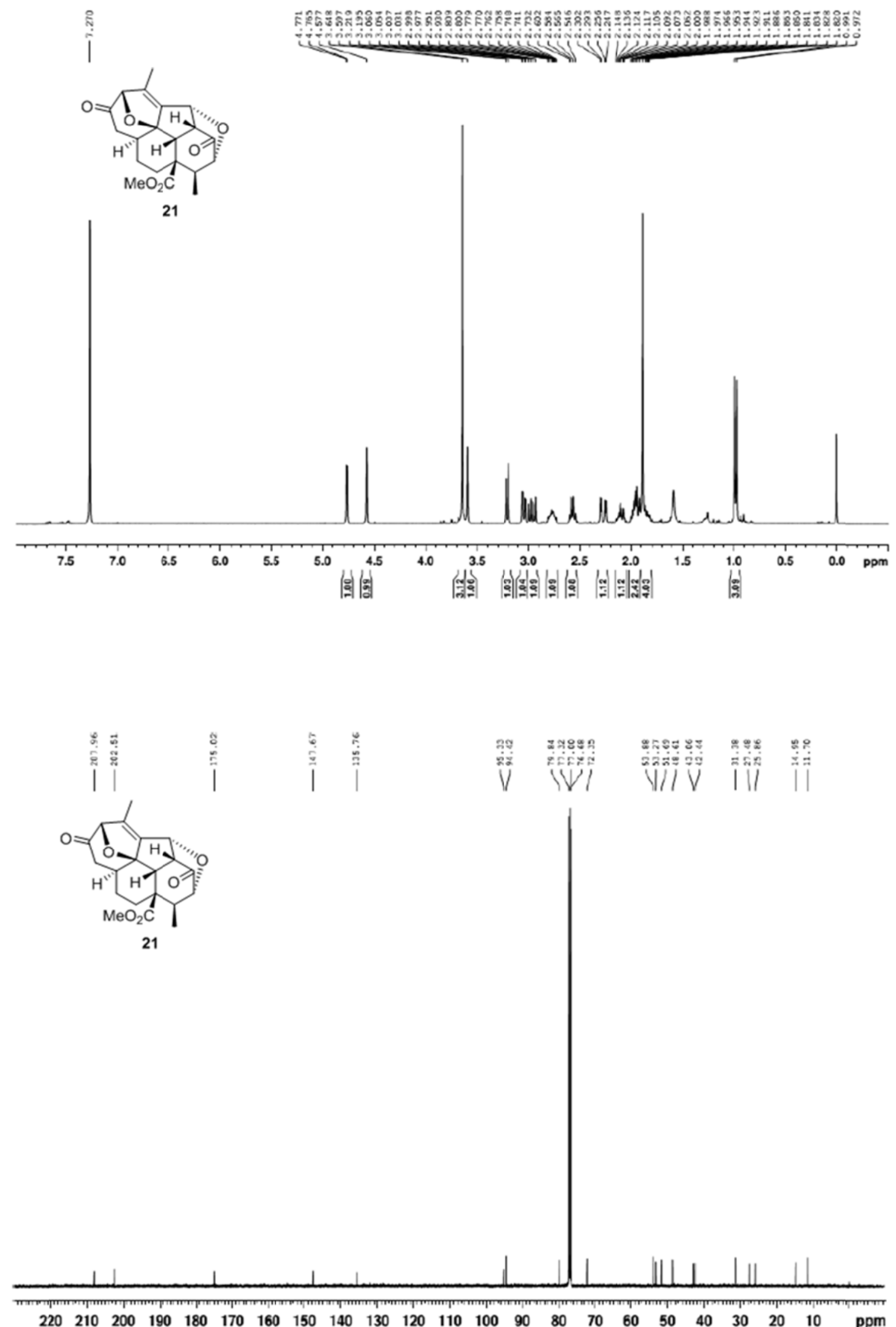

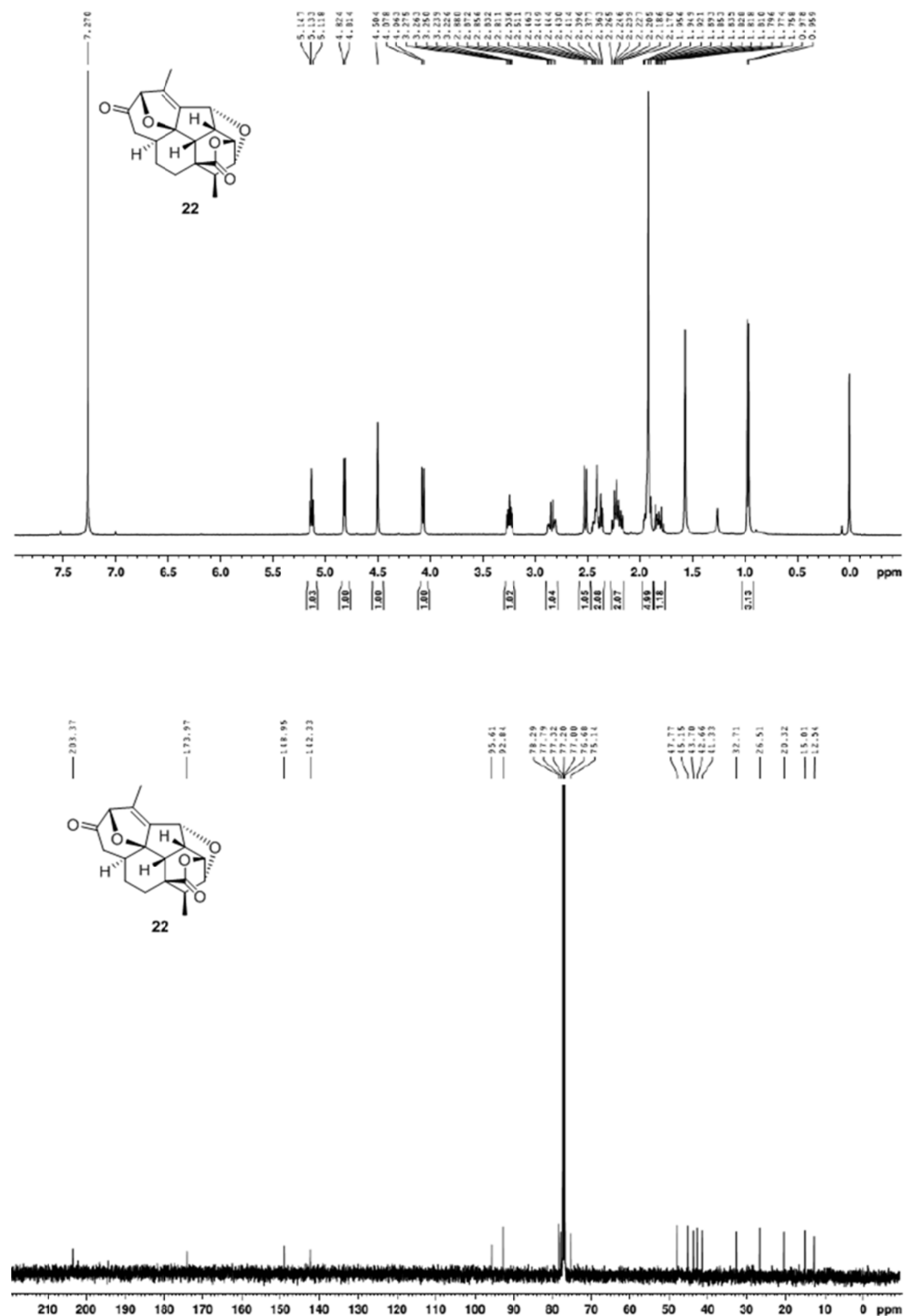

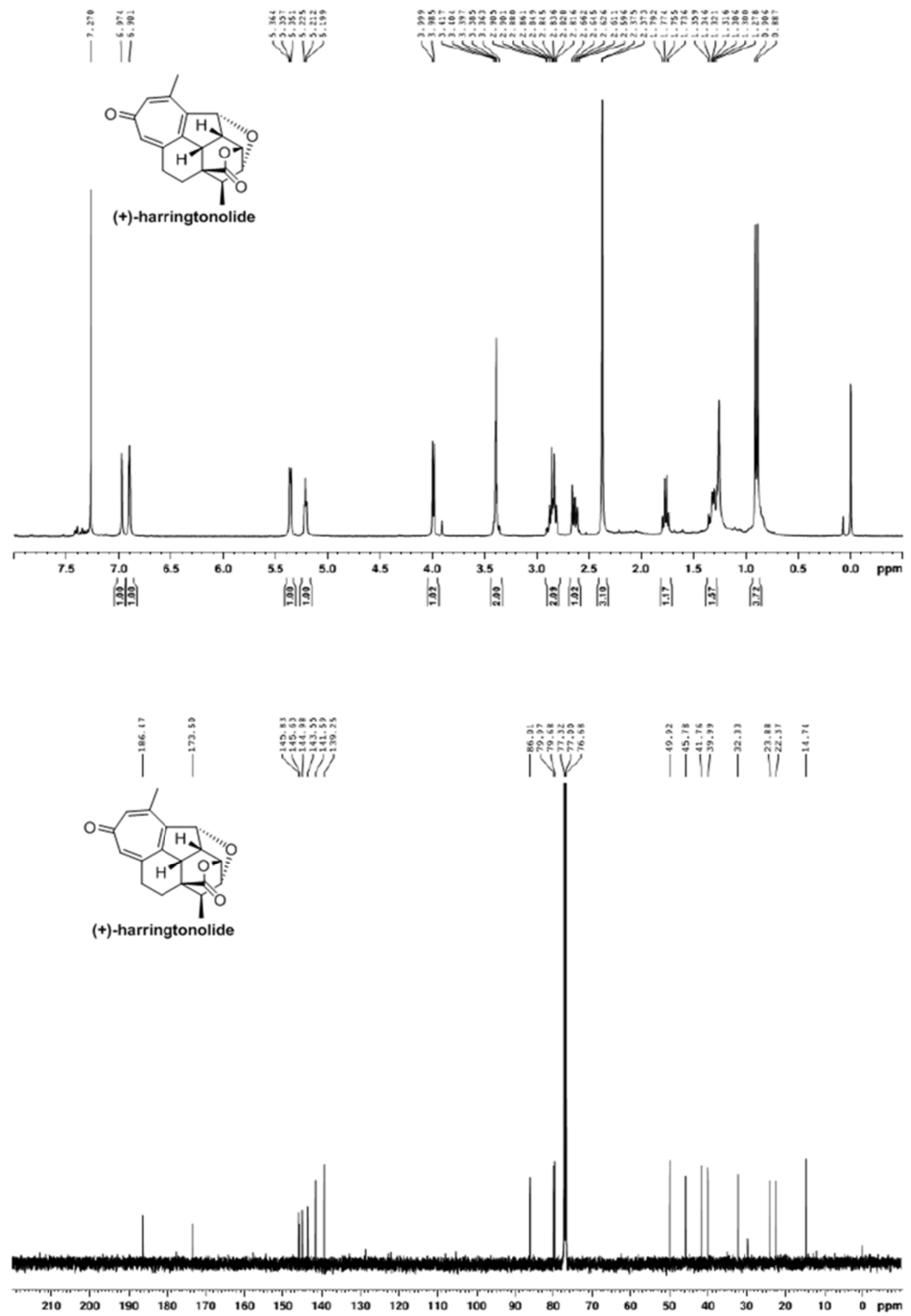

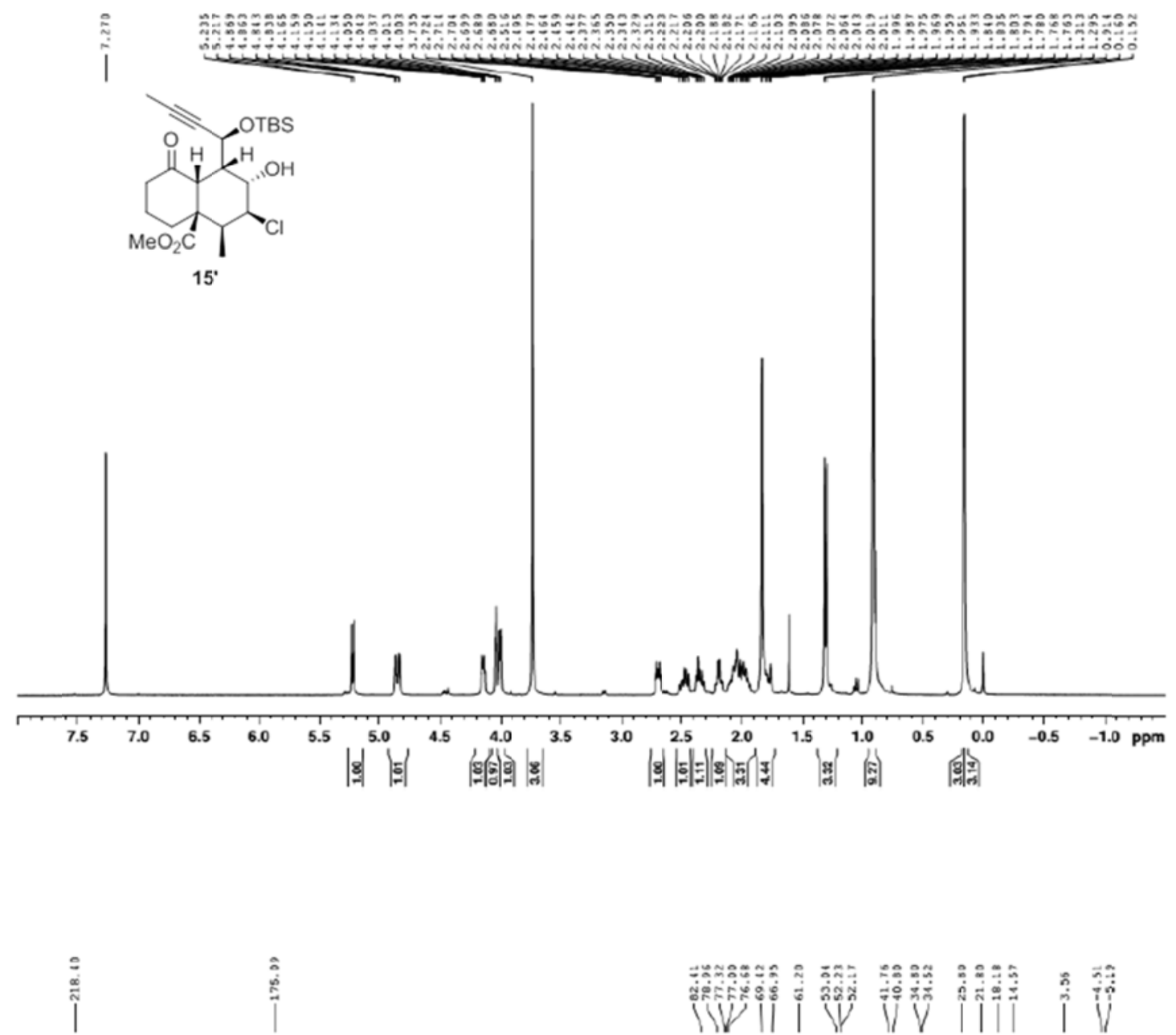

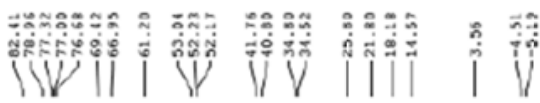
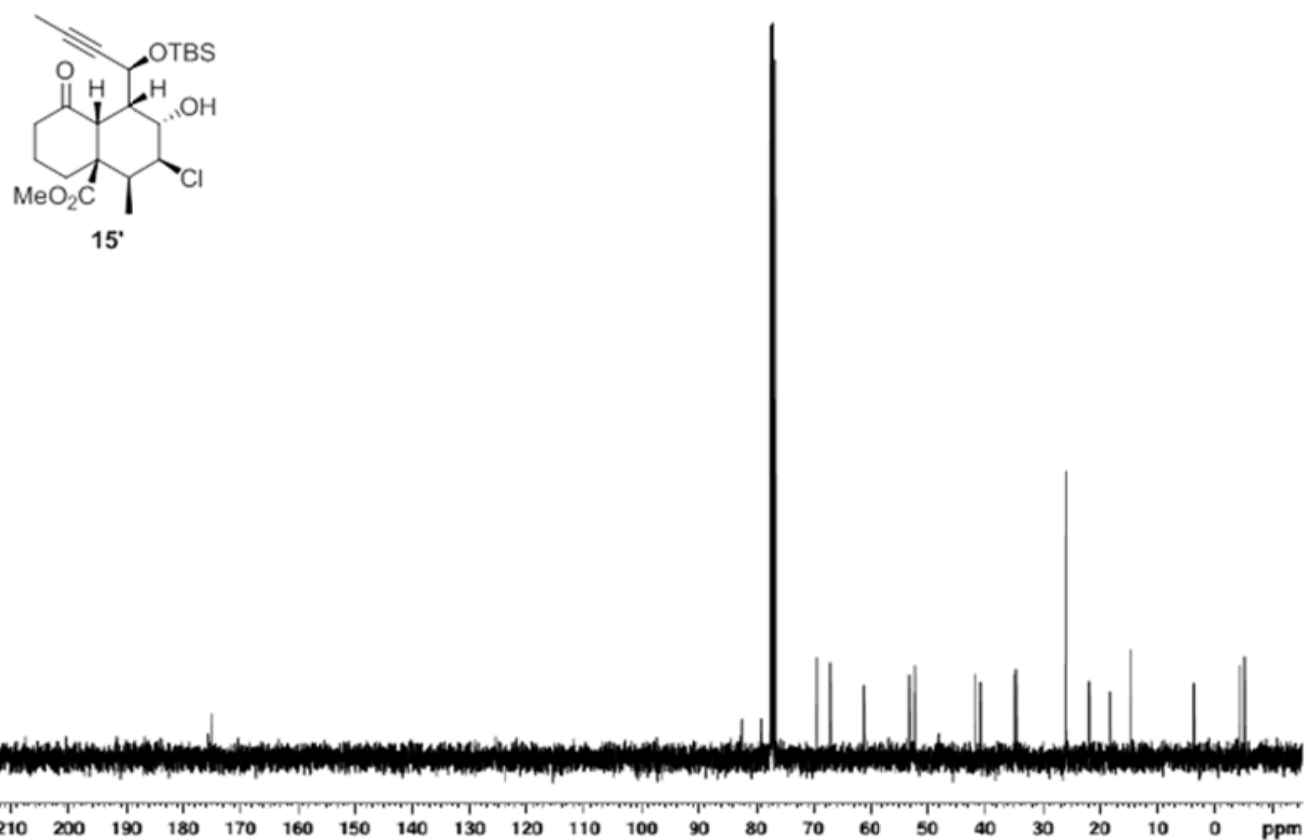

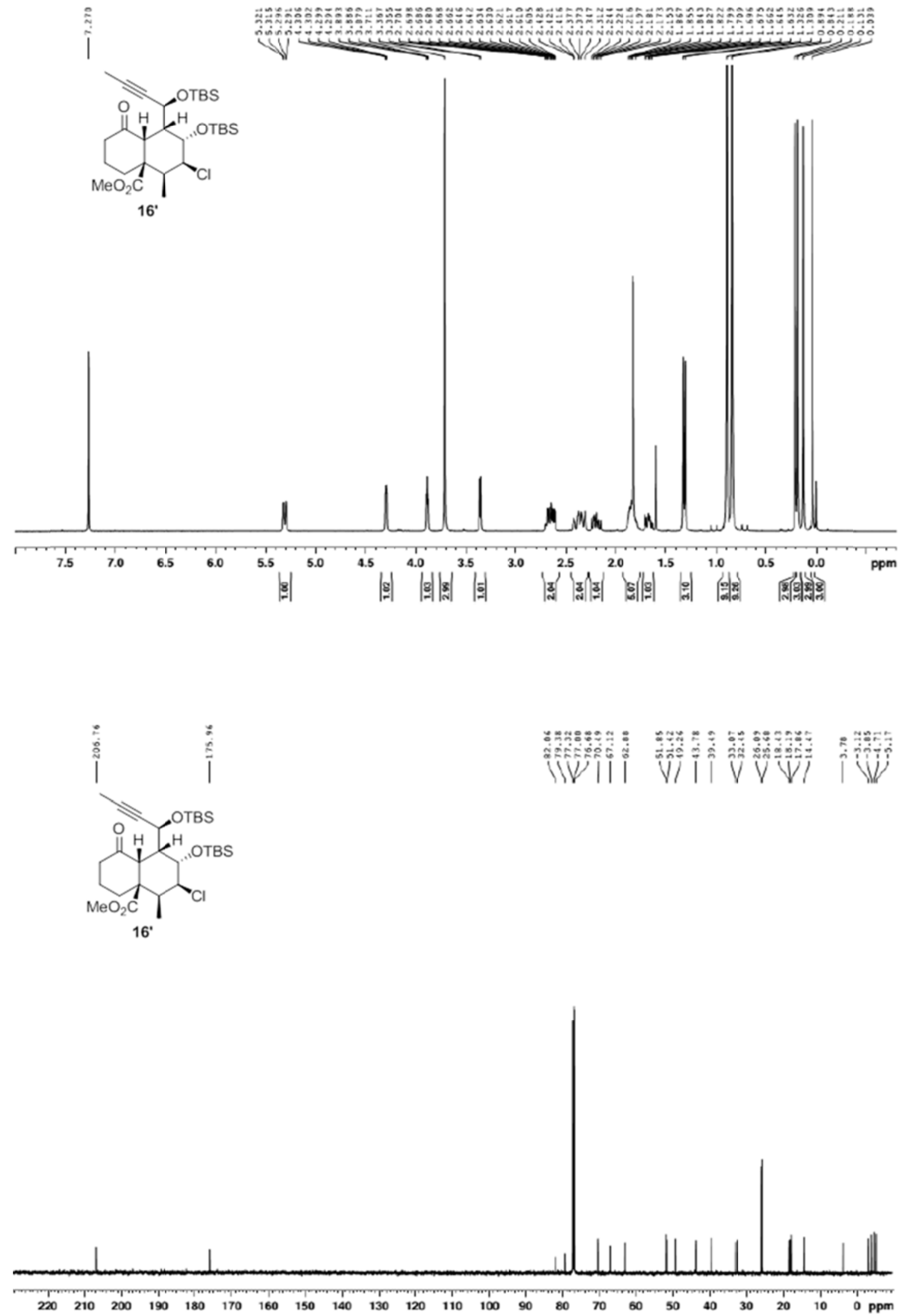

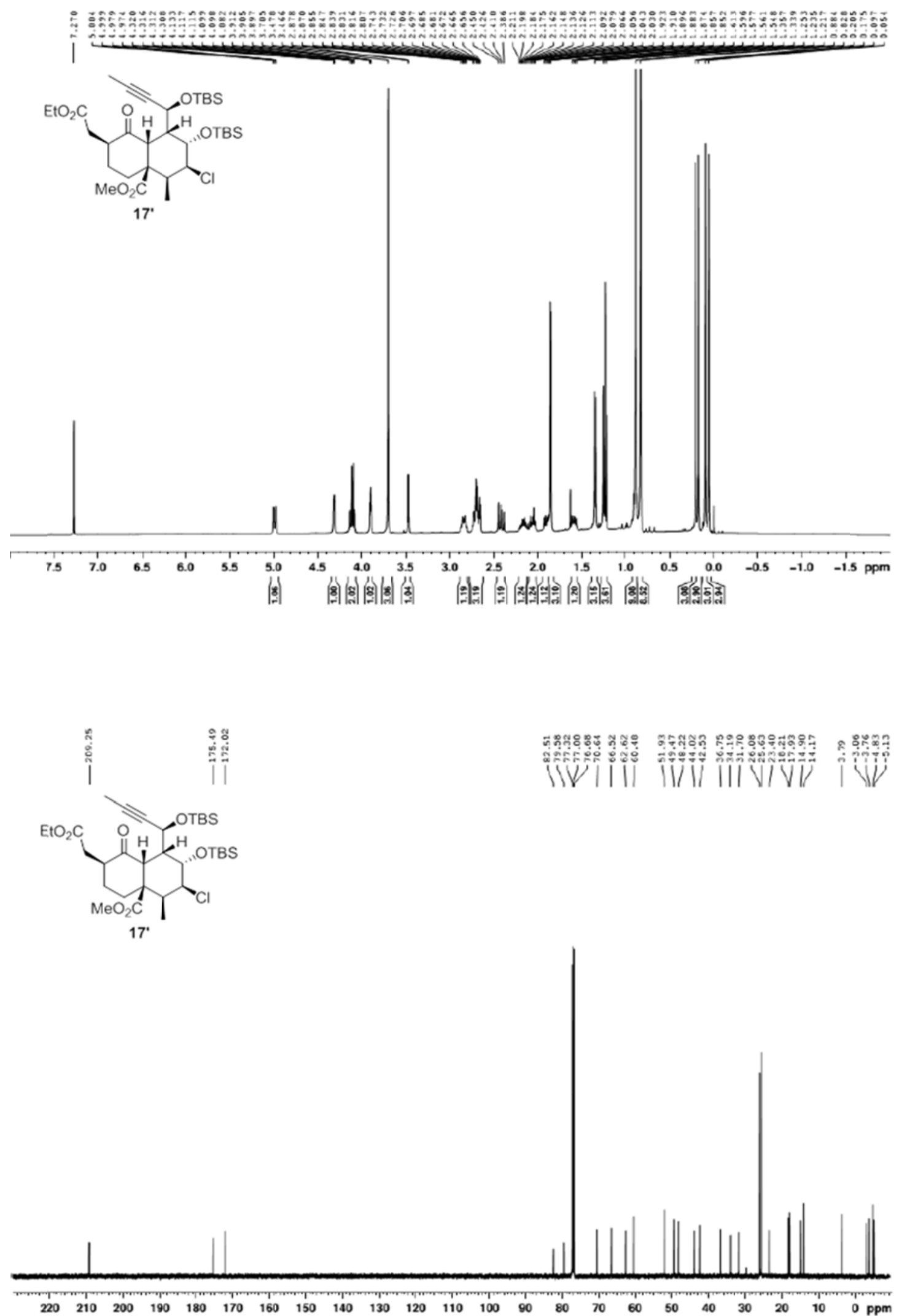

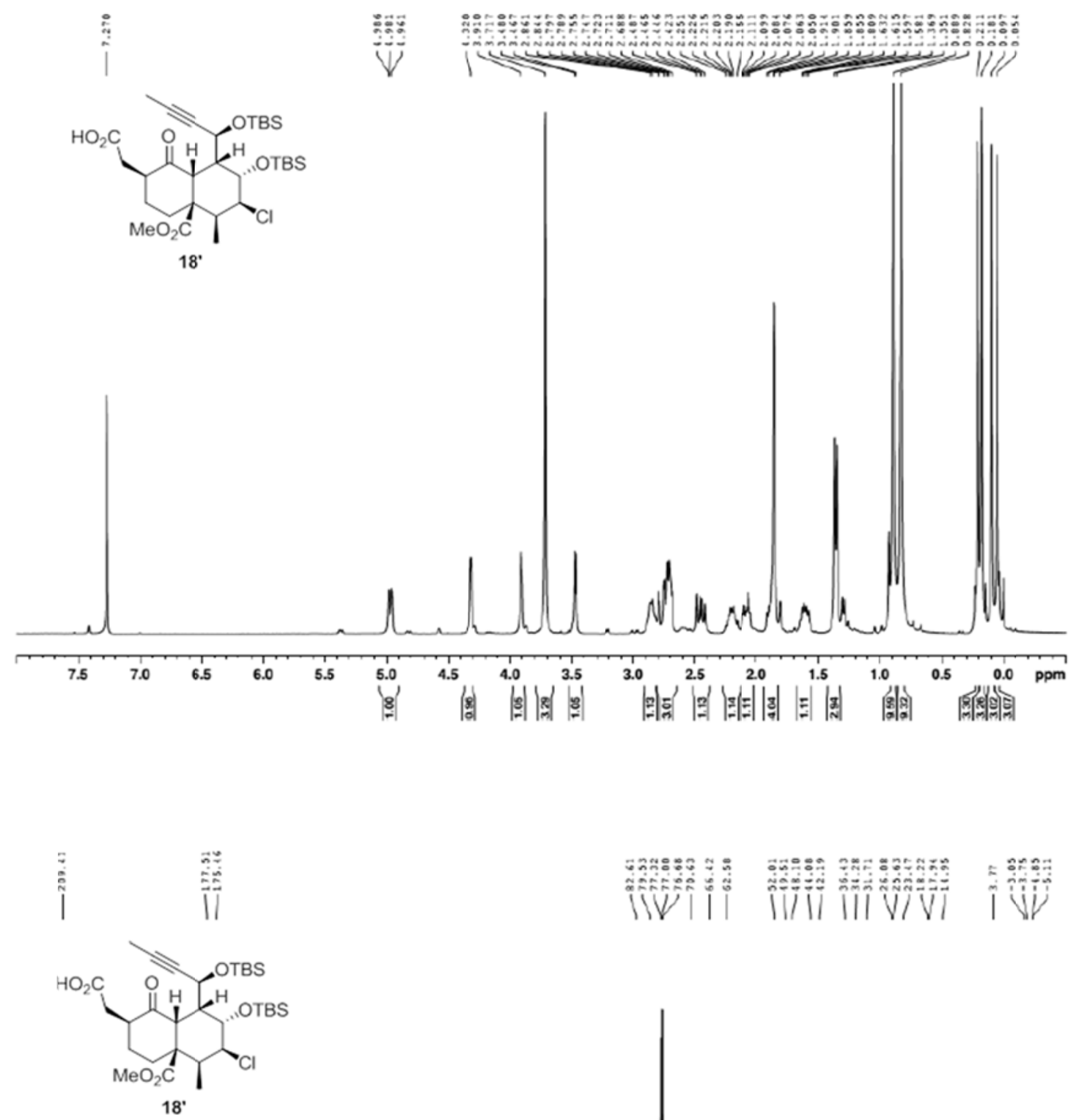

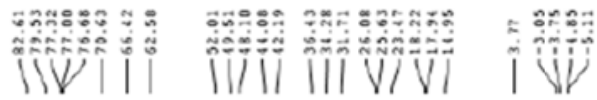
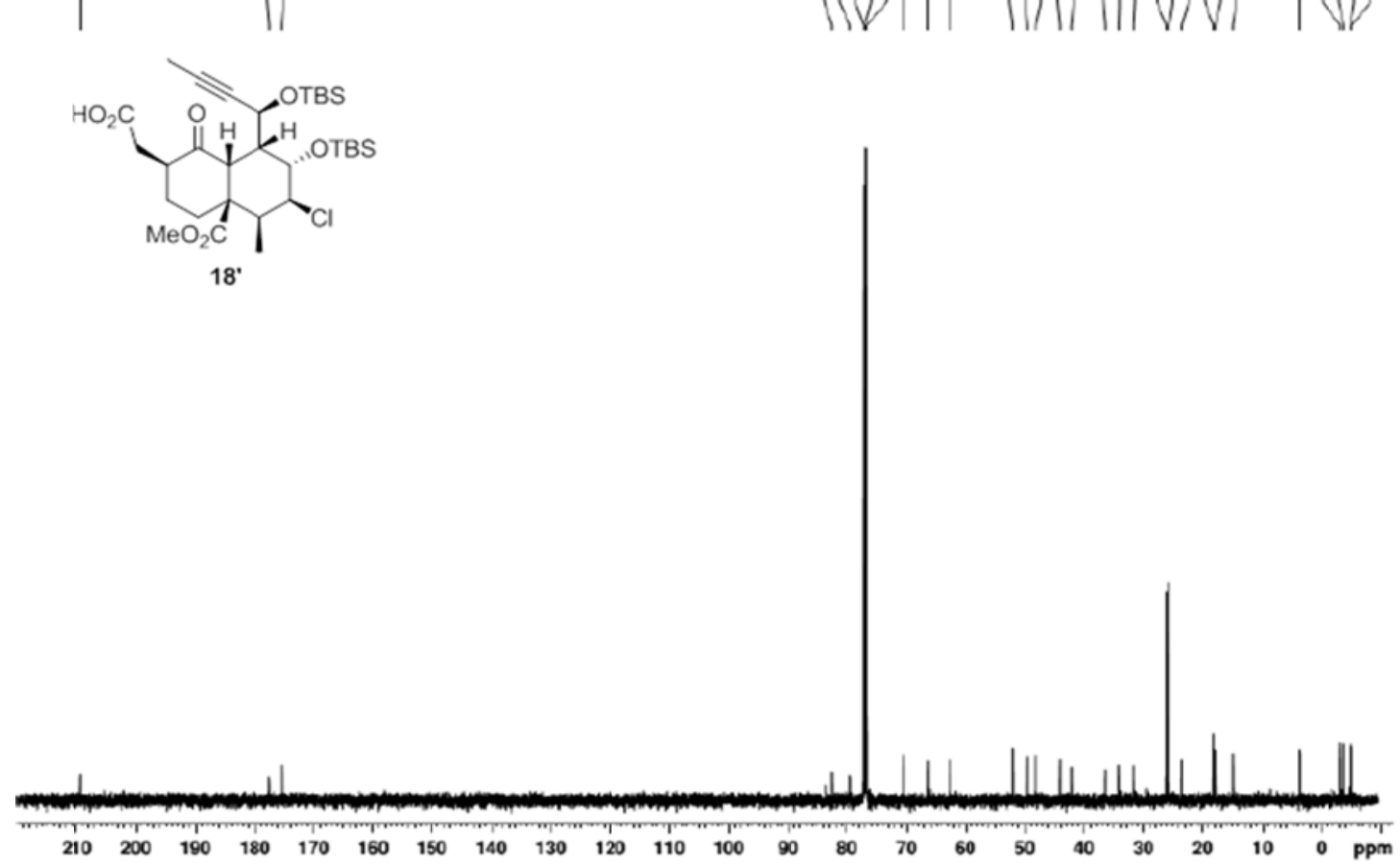
ลิ

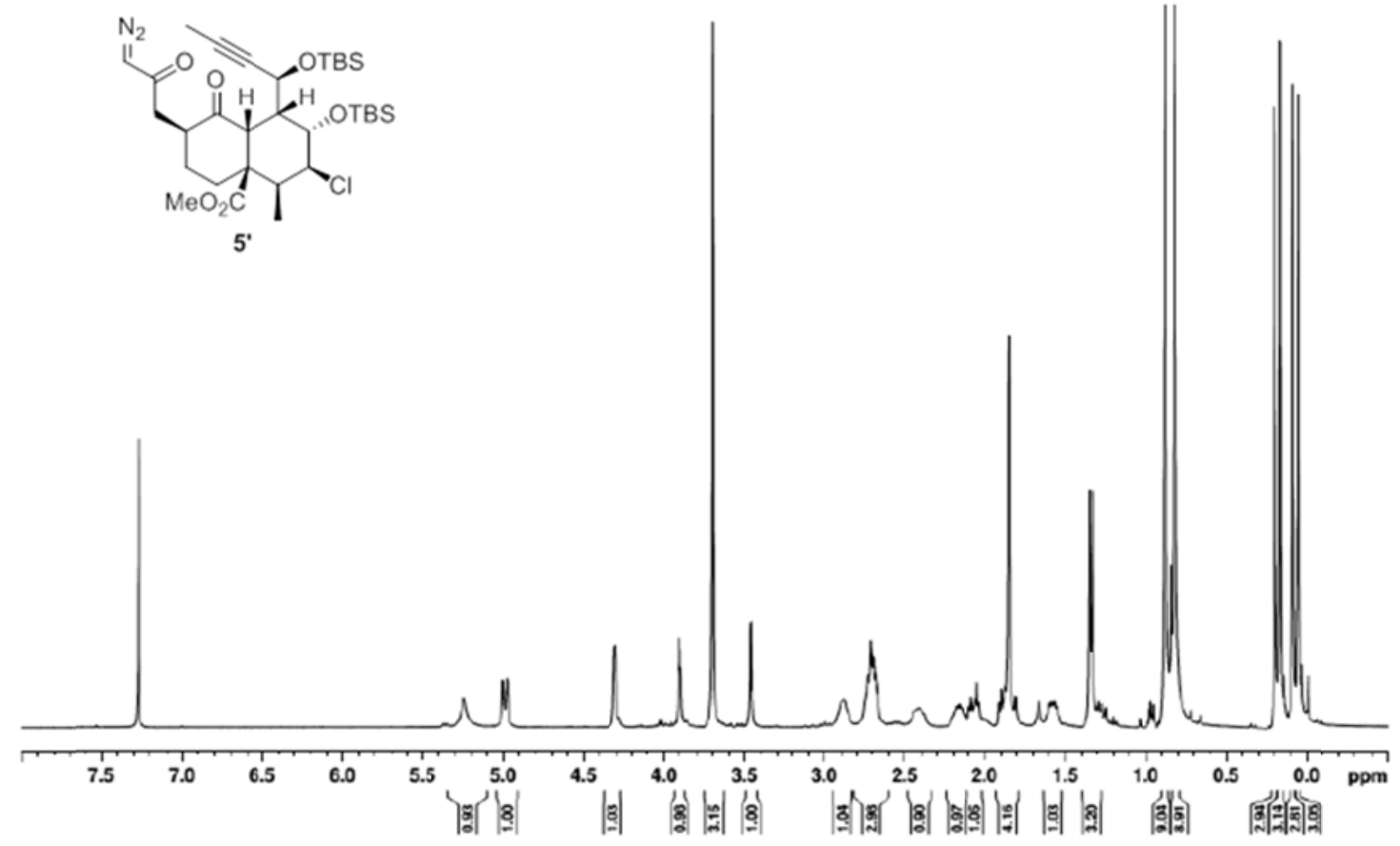

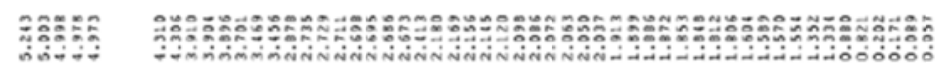

|

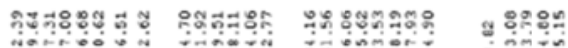

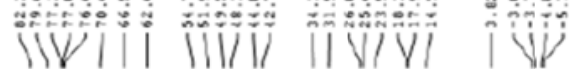
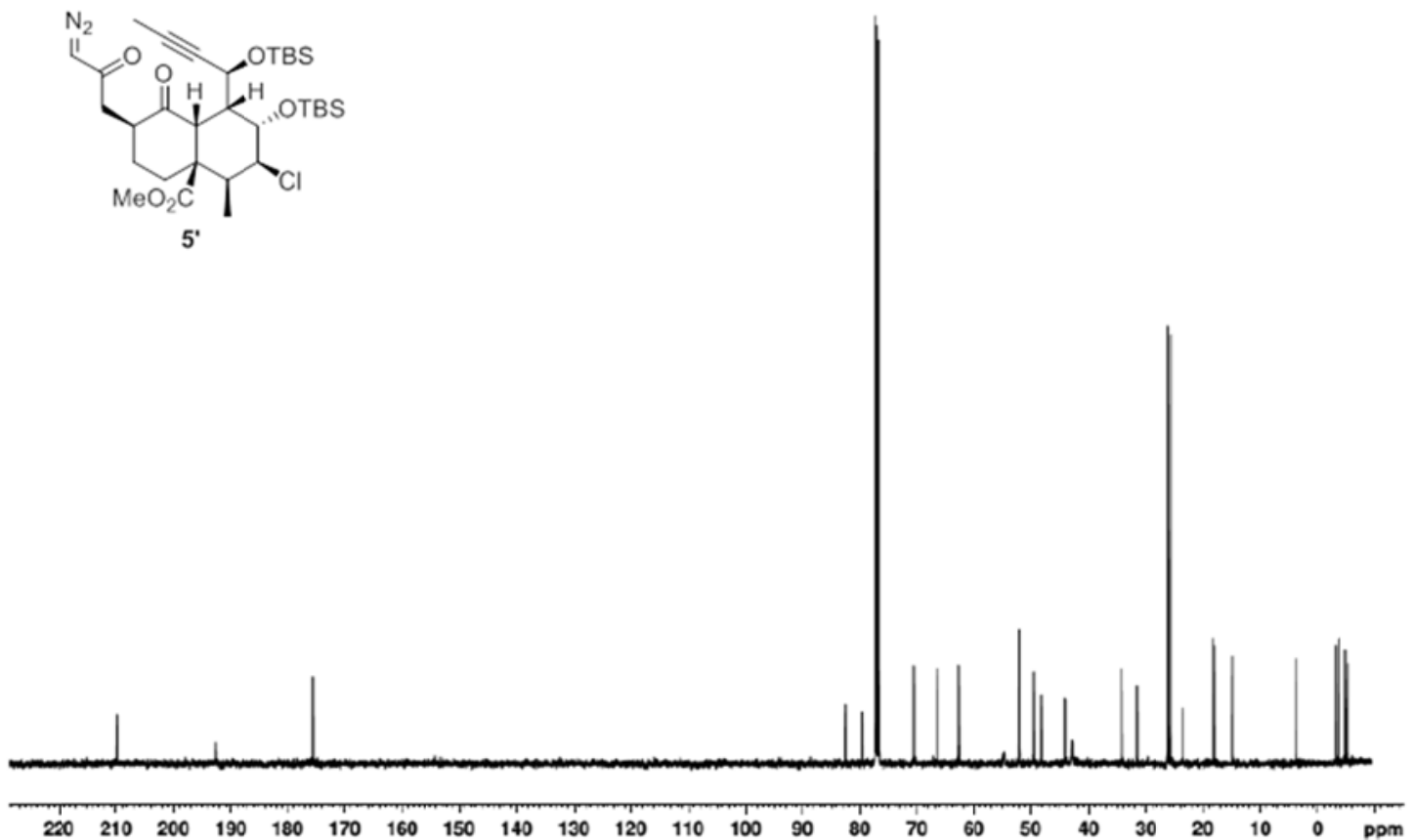


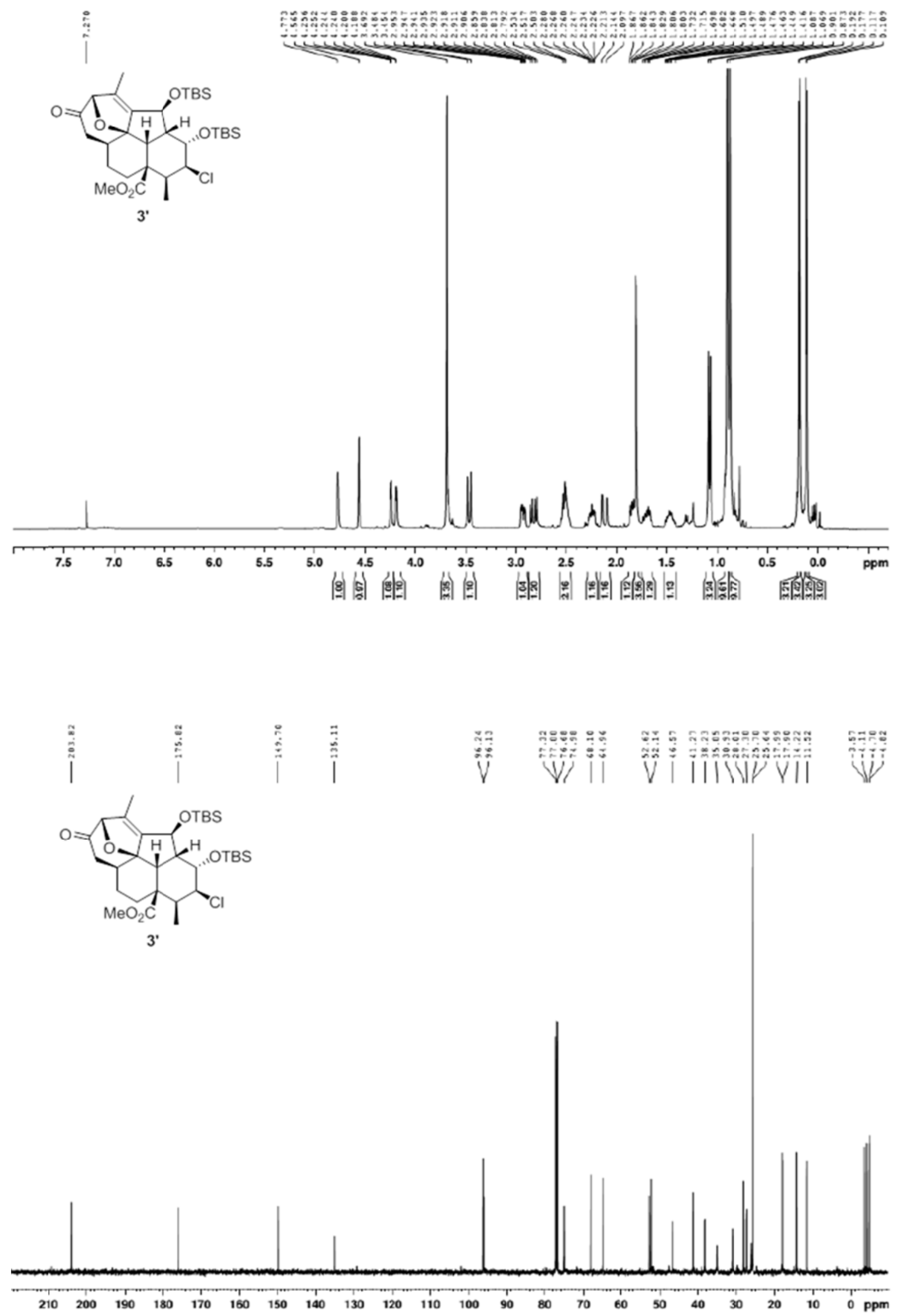




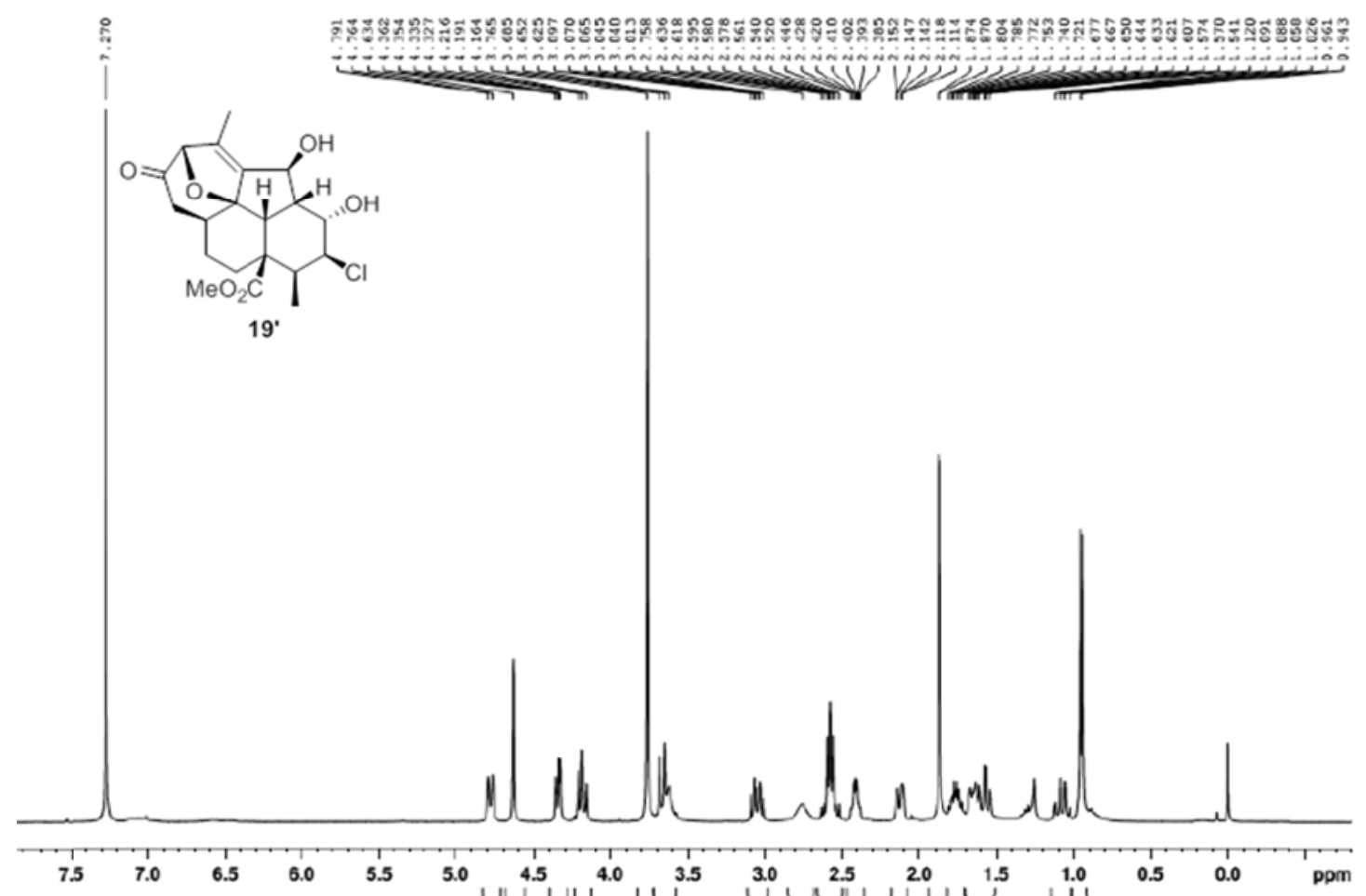

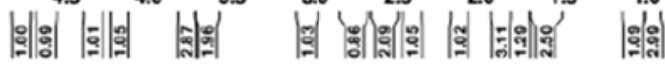
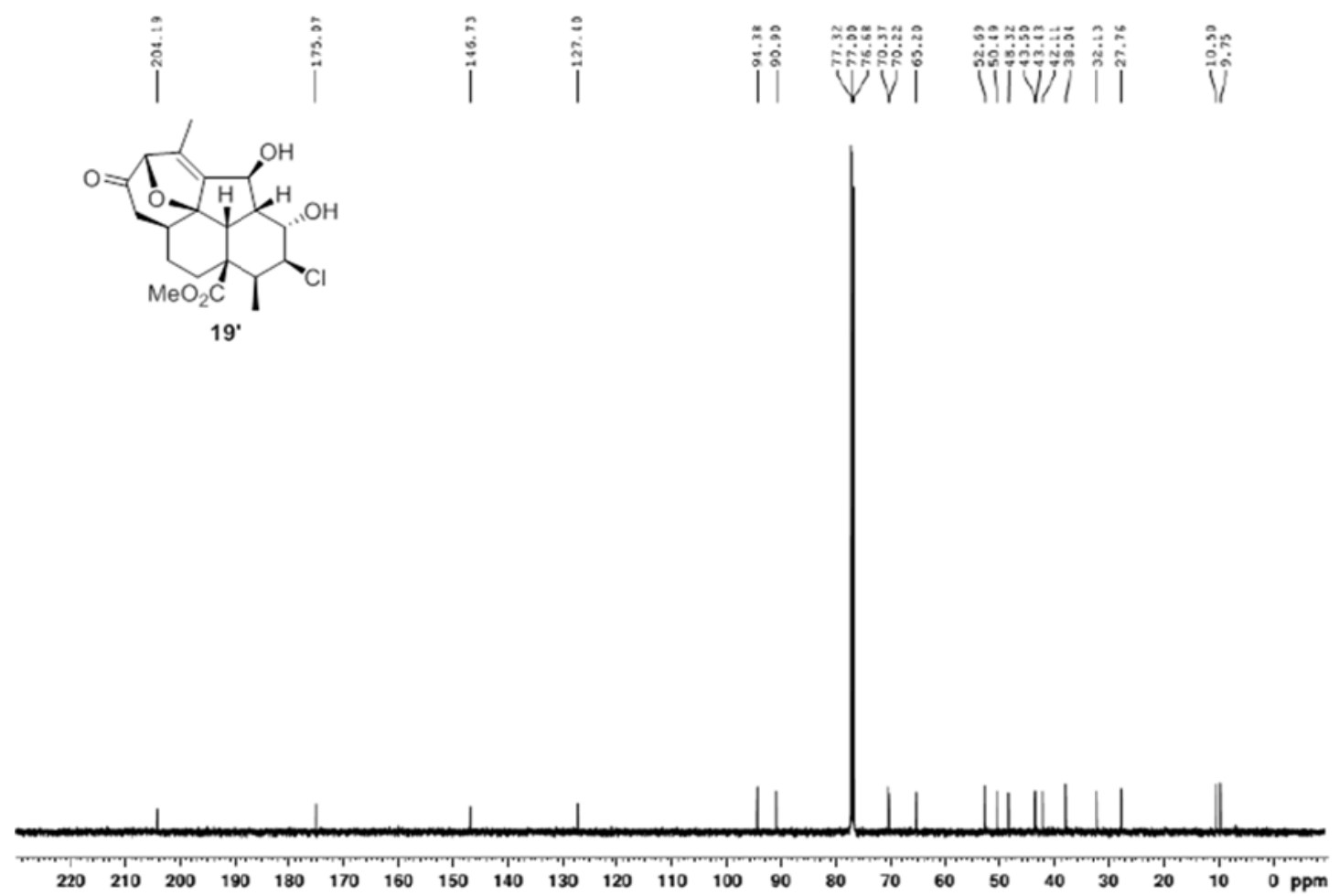

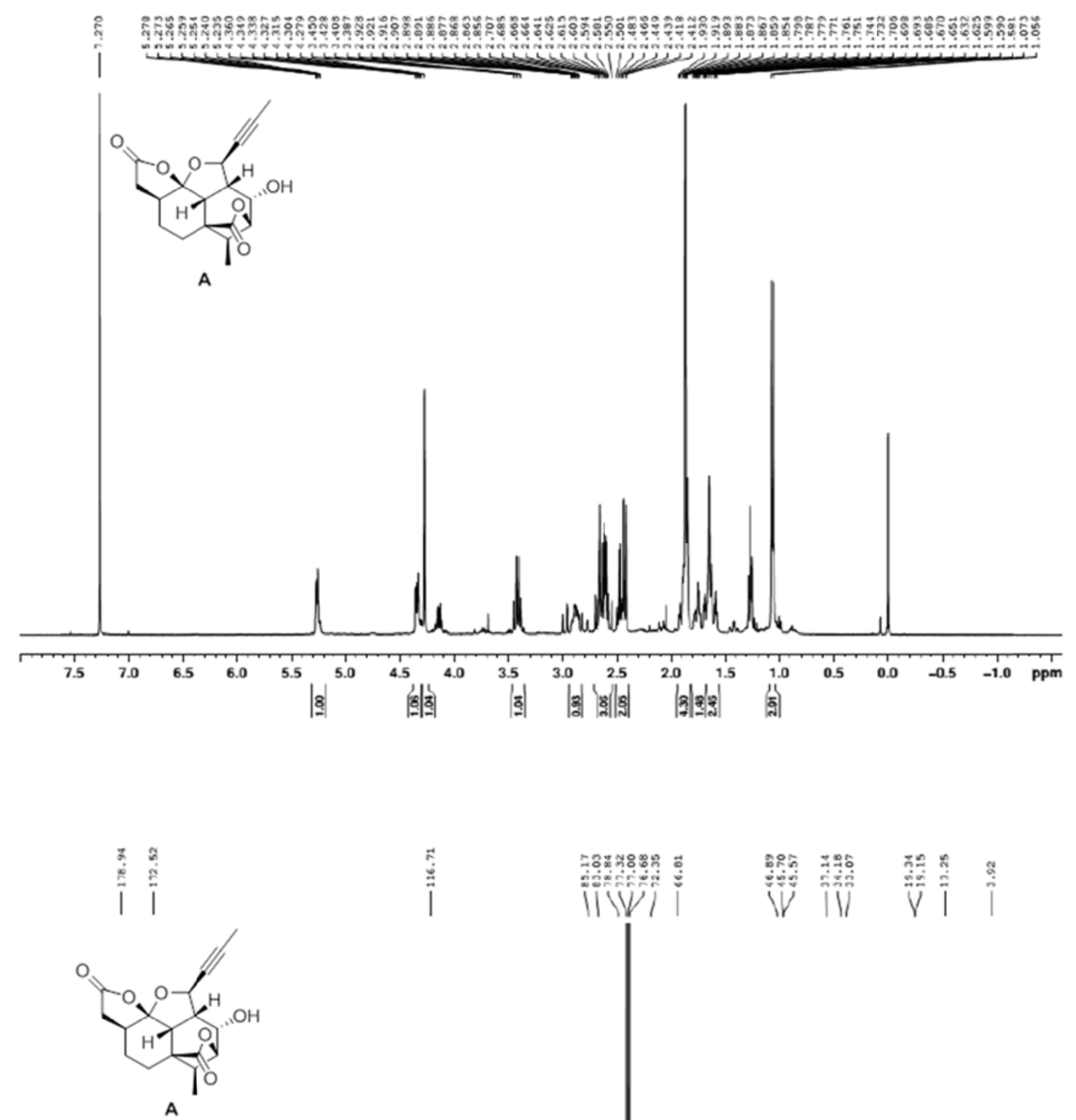

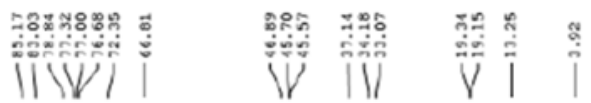

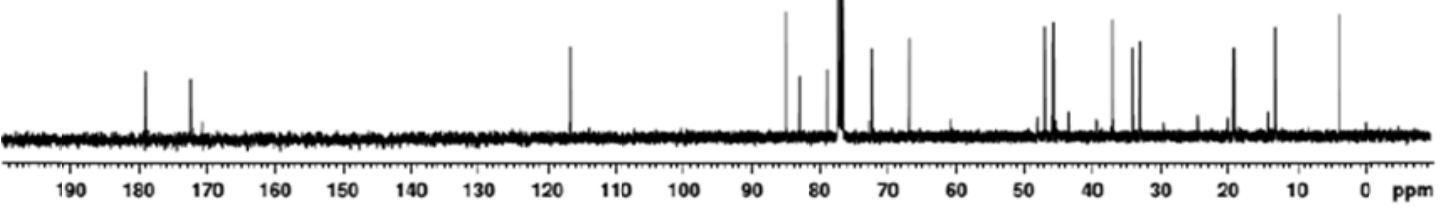



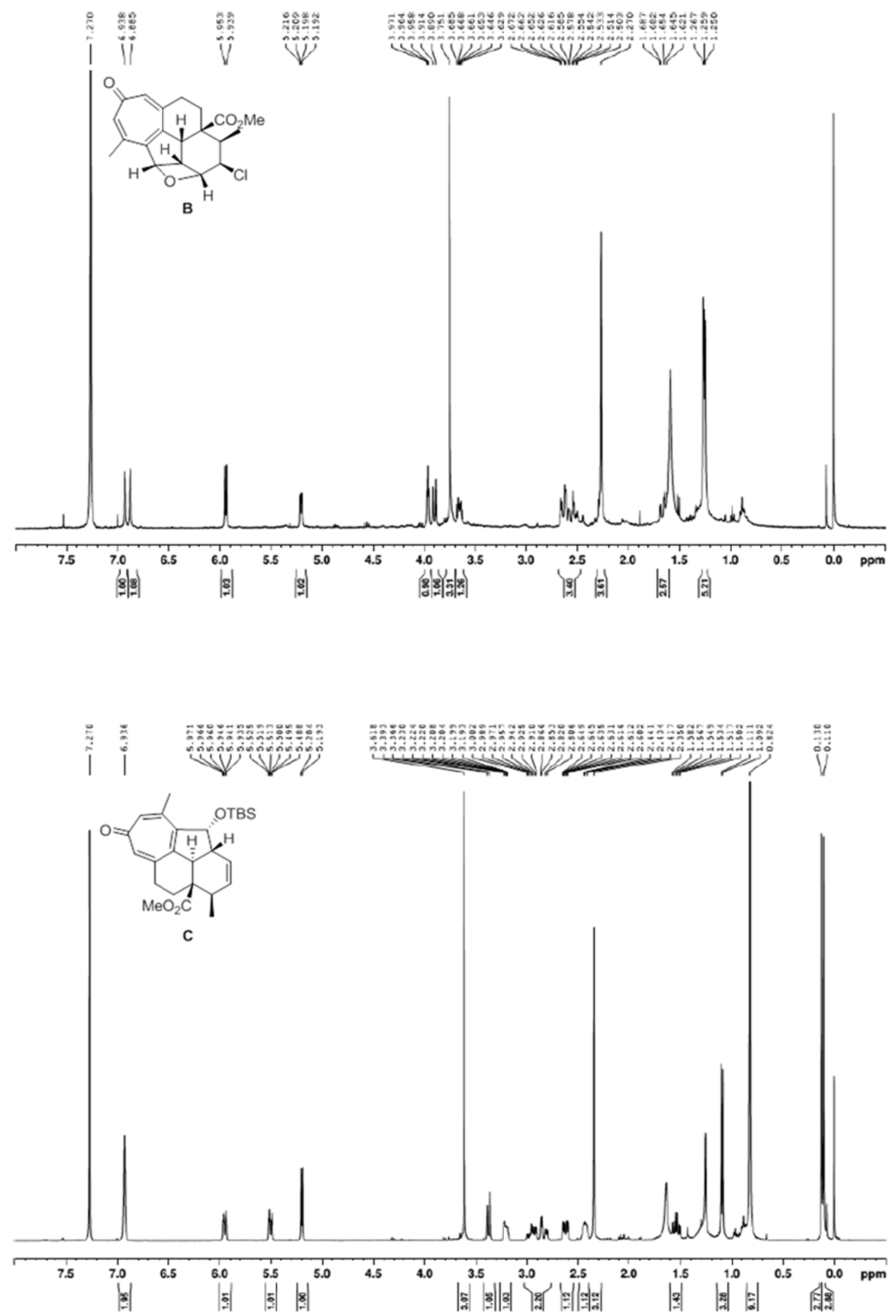

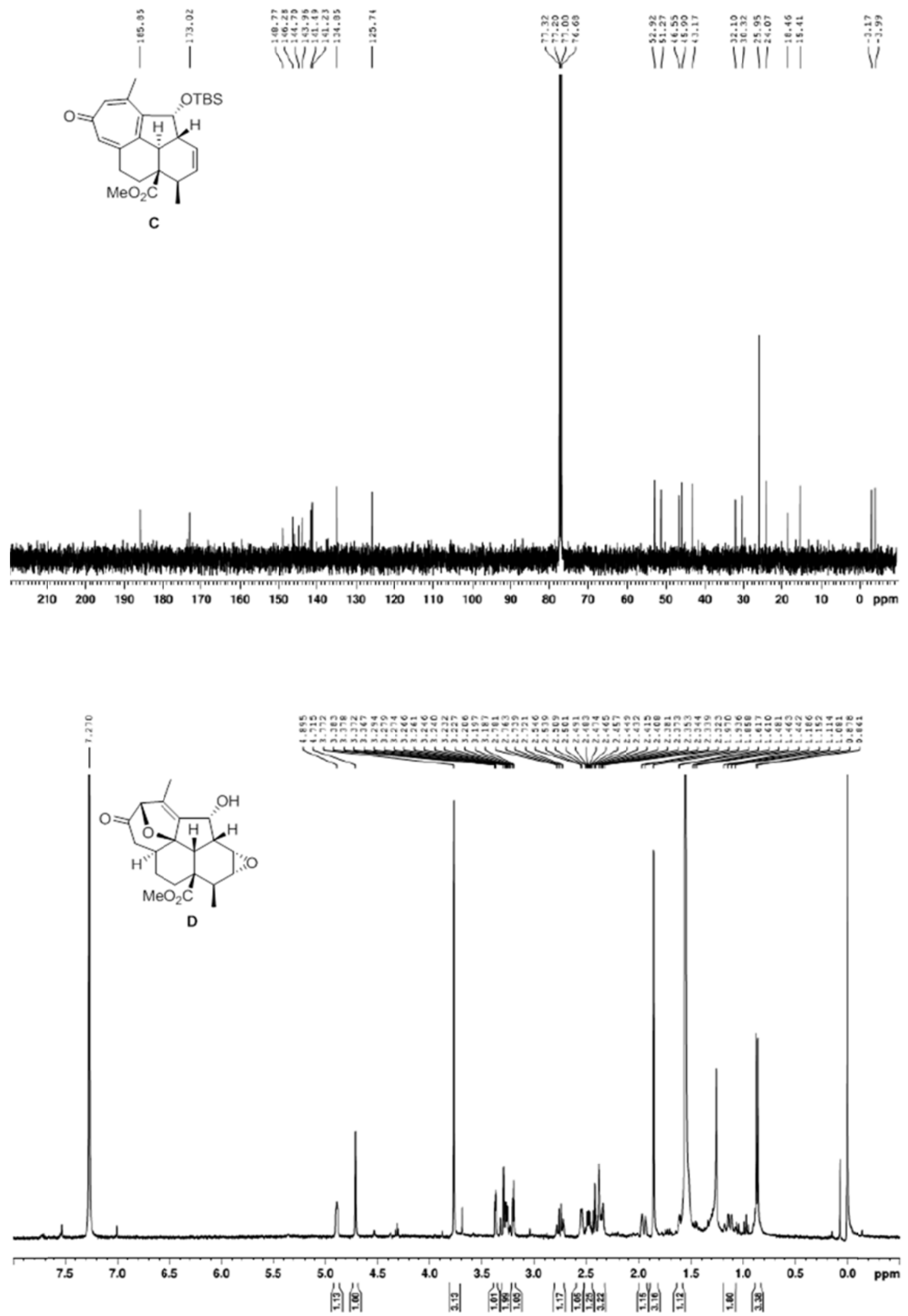

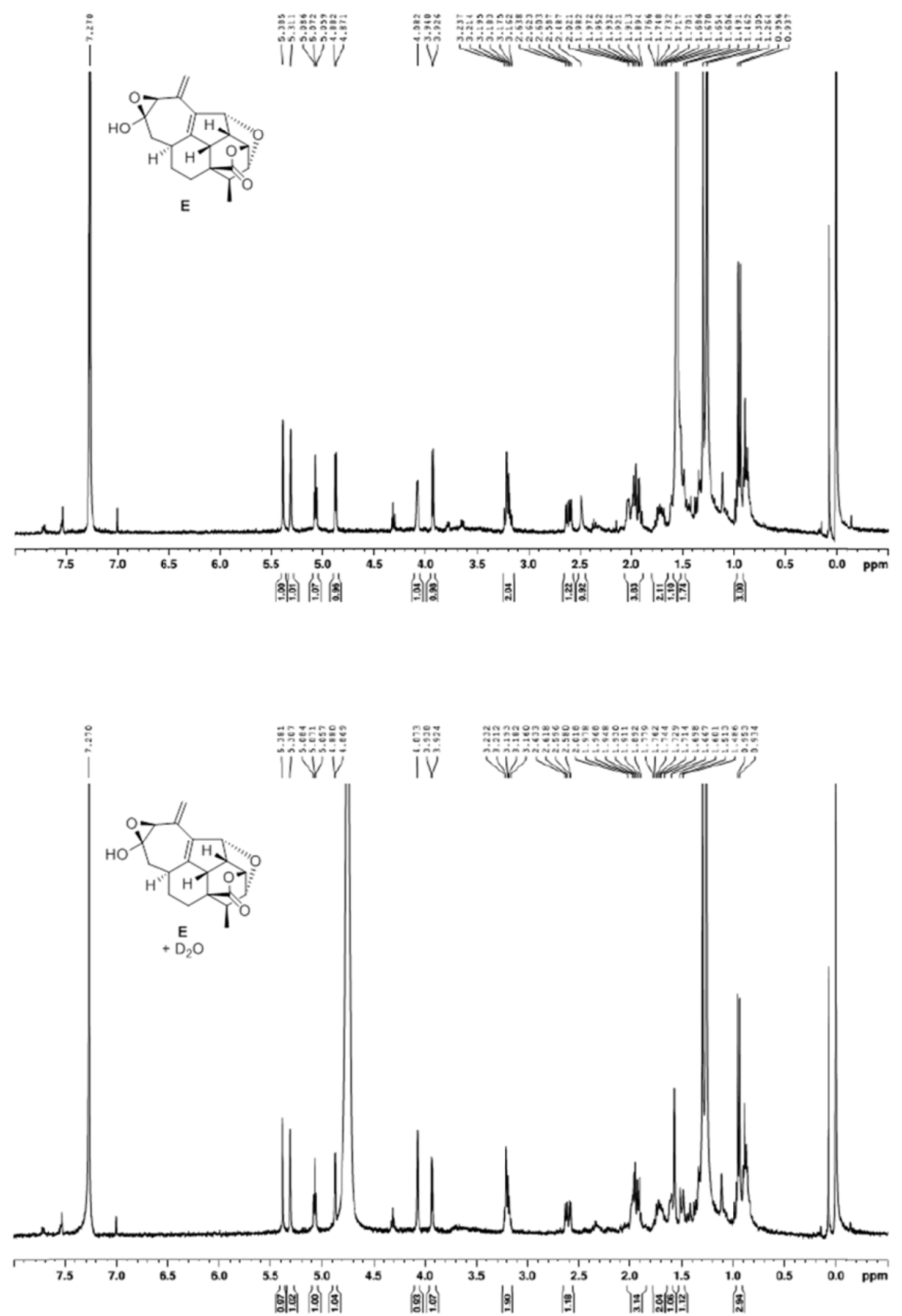

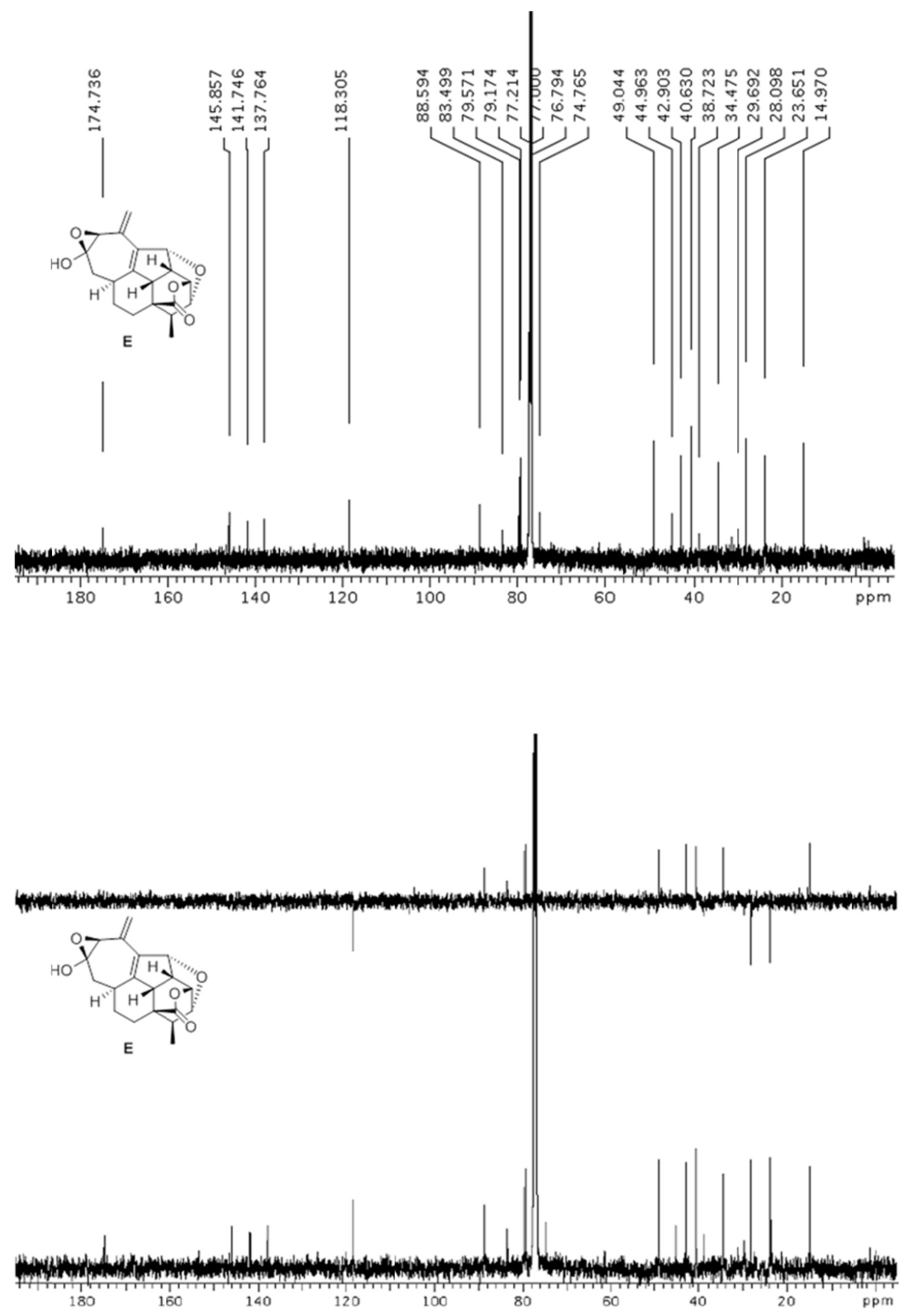